\title{
An overview of Booster and AGS polarized proton operations during Run 17
}

\author{
K. Zeno
}

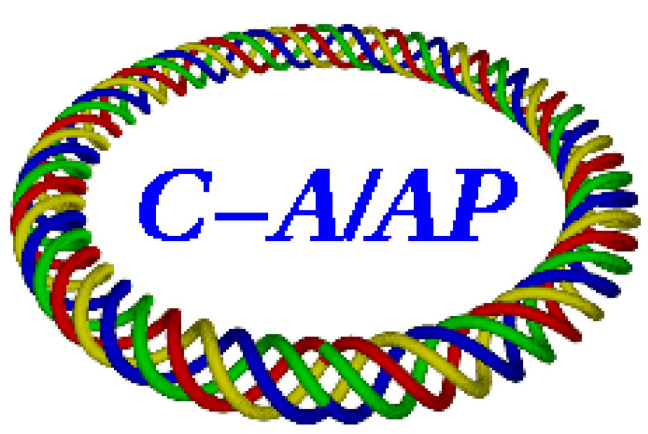

Collider-Accelerator Department Brookhaven National Laboratory Upton, N.Y. 11973

\section{U.S. Department of Energy Office of Science, Office of Nuclear Physics}

Notice: This document has been authorized by employees of Brookhaven Science Associates, LLC under Contract No. DE-SC0012704 with the U.S. Department of Energy. The United States Government retains a non- exclusive, paid-up, irrevocable, world-wide license to publish or reproduce the published form of this document, or allow others to do so, for United States Government purposes. 


\section{DISCLAIMER}

This report was prepared as an account of work sponsored by an agency of the United States Government. Neither the United States Government nor any agency thereof, nor any of their employees, nor any of their contractors, subcontractors, or their employees, makes any warranty, express or implied, or assumes any legal liability or responsibility for the accuracy, completeness, or any third party's use or the results of such use of any information, apparatus, product, or process disclosed, or represents that its use would not infringe privately owned rights. Reference herein to any specific commercial product, process, or service by trade name, trademark, manufacturer, or otherwise, does not necessarily constitute or imply its endorsement, recommendation, or favoring by the United States Government or any agency thereof or its contractors or subcontractors. The views and opinions of authors expressed herein do not necessarily state or reflect those of the United States Government or any agency thereof. 


\section{An Overview of Booster and AGS Polarized Proton Operations during Run 17}

Keith Zeno

$10 / 11 / 2017$ 


\section{Introduction}

There were only a few differences in the setup between this year's Polarized Proton run and the previous one (Run 15). ${ }^{1}$ Consequently, this note will focus on these differences as well as a few more notable studies done during the course of the run.

This year, the Booster input intensity was kept around 7e11 for the majority of the run whereas in Run 15 it was kept around 9e11. It was lowered because there was some indication that the source polarization was higher with this lower input. ${ }^{2}$ Some of the polarization measurements that motivated this change will be discussed.

Both the emittance and polarization on the AGS flattop show intensity dependence, thought to be related to the peak current, especially early in the AGS acceleration ramp. In Run 15 , the AGS Rf was configured for $\mathrm{h}=8$, but in this run $\mathrm{h}=6$ was used to reduce the peak current and also to allow for the possibility of using a dual harmonic to reduce it further. Eventually, a dual harmonic configuration was used for the first $100 \mathrm{~ms}$ or so of the AGS acceleration cycle. Two cavities were set to $\mathrm{h}=12$ and phased differently than the other 8 to accomplish this. Quad pumping was also used at Booster extraction to make the bunch injected into the AGS wider in order to match the dual harmonic bucket right at injection. This configuration, which was used for the majority of the run, will be described. Measurements of the intensity dependence of the transverse emittance and polarization with and without it will be compared.

There was also a study performed in Run 16 with a 'bare' AGS machine (i.e.- no snakes, jump quads, etc.). The transverse emittance and its intensity dependence are compared with the data for the dual harmonic PP setup from this run.

Another possible way to reduce this dependence was proposed: It is to capture the Linac pulse into two bunches of half intensity instead of 1 full intensity bunch in the Booster and merge them on the AGS flattop. ${ }^{3}$ In order to accomplish this it was necessary to develop a 'bucket switch' in the Booster. This 'bucket switch' (for lack of a better name) consists of moving the two bunches in $\mathrm{h}=2$ buckets into two of the three $\mathrm{h}=3$ buckets in the Booster so that they can be injected into adjacent $\mathrm{h}=12$ AGS buckets.

The vertical sextupole current required to produce near zero vertical chromaticity was much greater this run than in the past. The reason for this still remains a mystery, but many beam based measurements were taken and this note will describe them.

Several studies on the effect of the $\mathrm{H}^{-}$stripping foil on transverse emittance were performed. The results of these will be discussed and compared with each other. The effect on

\footnotetext{
1 “An Overview if Booster and AGS Polarized Proton Operation During Run 15”, K. Zeno, describes the Run 15 setup in some detail.

${ }^{2}$ See H. Huang's presentation at the RHIC retreat about the injectors, slide 13.

${ }^{3} \mathrm{H}$. Huang, private communication
} 
transverse emittance of scraping at injection vs. scraping later in the cycle was also investigated and the results will be described. Transverse emittance measurements in BtA and on the AGS flattop will be described and compared with similar data from Run 15. Measurements performed throughout the run of the longitudinal emittance on the flattop are also summarized.

\section{Scraping at Booster Injection vs. Later in the Cycle}

Early in the run a couple of studies were performed to determine the relative effectiveness of scraping at injection vs. the normal scraping setup. To scrape horizontally at injection the dump bump was used, and to scrape vertically a 3-bump at E7 was used. The initial tests were done in December (see Table I). Gaussian fits of the profiles from BtA MW006 were used to measure the $95 \%$ normalized transverse emittances in each case. The full widths at half max of the unscraped beam at MW006 are about $(\Delta x, \Delta y)=(4,7) \mathrm{mm}$.

From Table I, it seems that the late horizontal scrape has very little coupling to the vertical and that the other scrapes are significantly coupled. For this study the smallest $\varepsilon_{\text {avg }}$ was obtained with the vertical scrape later in the cycle, with a horizontal scrape at injection only slightly larger, and that difference is likely well within the error. The horizontal scrape at injection, without any other scraping, was used for a few days in early January. When scraping (in either plane) at injection is used the vertical profile develops tails that are not present when vertical scraping late in the cycle is used (see Figure 1).

\begin{tabular}{|l|c|c|c|c|c|}
\hline \multicolumn{1}{|c|}{ Scraping Method } & $\boldsymbol{\Delta x}$ & $\boldsymbol{\Delta y}$ & $\boldsymbol{\varepsilon}_{\mathbf{x}}$ & $\boldsymbol{\varepsilon}_{\mathbf{y}}$ & $\boldsymbol{\varepsilon}_{\mathbf{a v g}}$ \\
\hline Vertical @ injection & 3.48 & 4.49 & 9.93 & 3.12 & 7.36 \\
\hline Horizontal @ injection & 2.82 & 5.73 & 6.52 & 5.09 & 5.85 \\
\hline Vertical later in cycle & 3.09 & 3.97 & 7.83 & 2.44 & 5.79 \\
\hline Horizontal later in cycle & 2.41 & 6.93 & 4.76 & 7.44 & 6.25 \\
\hline
\end{tabular}

Table I: Transverse normalized 95\% emittances from BtA MW006 for 2.15e11 Booster Late for horizontal and vertical scraping at injection and later in the cycle. $\Delta \mathrm{x}$ and $\Delta \mathrm{y}$ are the full widths at half maximum of a Gaussian fit in $\mathrm{mm}, \boldsymbol{\varepsilon}_{\mathbf{x}}$ and $\boldsymbol{\varepsilon}_{\mathbf{y}}$ are the $95 \%$ normalized transverse emittances in $\pi \mathrm{mm}$ mrad, and $\varepsilon_{\text {avg }}$ is the root mean square of $\varepsilon_{\mathrm{x}}$ and $\varepsilon_{\mathrm{y}}{ }^{4}$

On January 9, the emittance of the horizontal scrape at injection was measured again, this time with about 2.6e11 at Booster late (also with somewhat lower Booster input than earlier, $\sim 7.2 \mathrm{e} 11$ vs. 7.8e11) and was compared to the case where both horizontal and vertical scraping later in the cycle were used. ${ }^{5}$ Table II shows the results. Scraping in both planes later in the cycle

\footnotetext{
${ }^{4}$ Booster-AGS-pp 2017 elog, Dec 222016 1356-1515 entries. The analysis in the elog uses vertical profiles that only fit their cores for the injection cases. Here I am fitting the entire vertical profile for all cases. The formulas $\varepsilon_{\mathrm{x}}=0.82 * \Delta \mathrm{x}^{2}$ and $\varepsilon_{\mathrm{y}}=0.155^{*} \Delta \mathrm{y}^{2}$ are used which give the $95 \%$ emittances for a Gaussian profile (see K. Zeno, "Booster and AGS Transverse Emittance During the 2006 and 2009 Polarized Proton Runs", pg. 3, C-A/AP\#404). ${ }^{5}$ Booster-AGS-PP 2017 elog Jan 9 2017, 1828 through 1835 entries. Once again the data for the injection horizontal scrape in the elog only fit the core of the vertical profile. Shown here is the data for a fit of an entire vertical profile.
} 
seems pretty clearly better than horizontal scraping at injection. The emittance on the flattop in the AGS (using the ion IPM) was also somewhat smaller in the vertical for the late scraping case, $\left(\varepsilon_{\mathrm{x}}, \varepsilon_{\mathrm{y}}\right)=(14,15.5)$ vs. $(14,17.5) \pi \mathrm{mm} \mathrm{mr}$ (AGS late intensity, which is measured about $8 \mathrm{~ms}$ before extraction time at At0+920 ms, was about 2.25e11). ${ }^{6}$
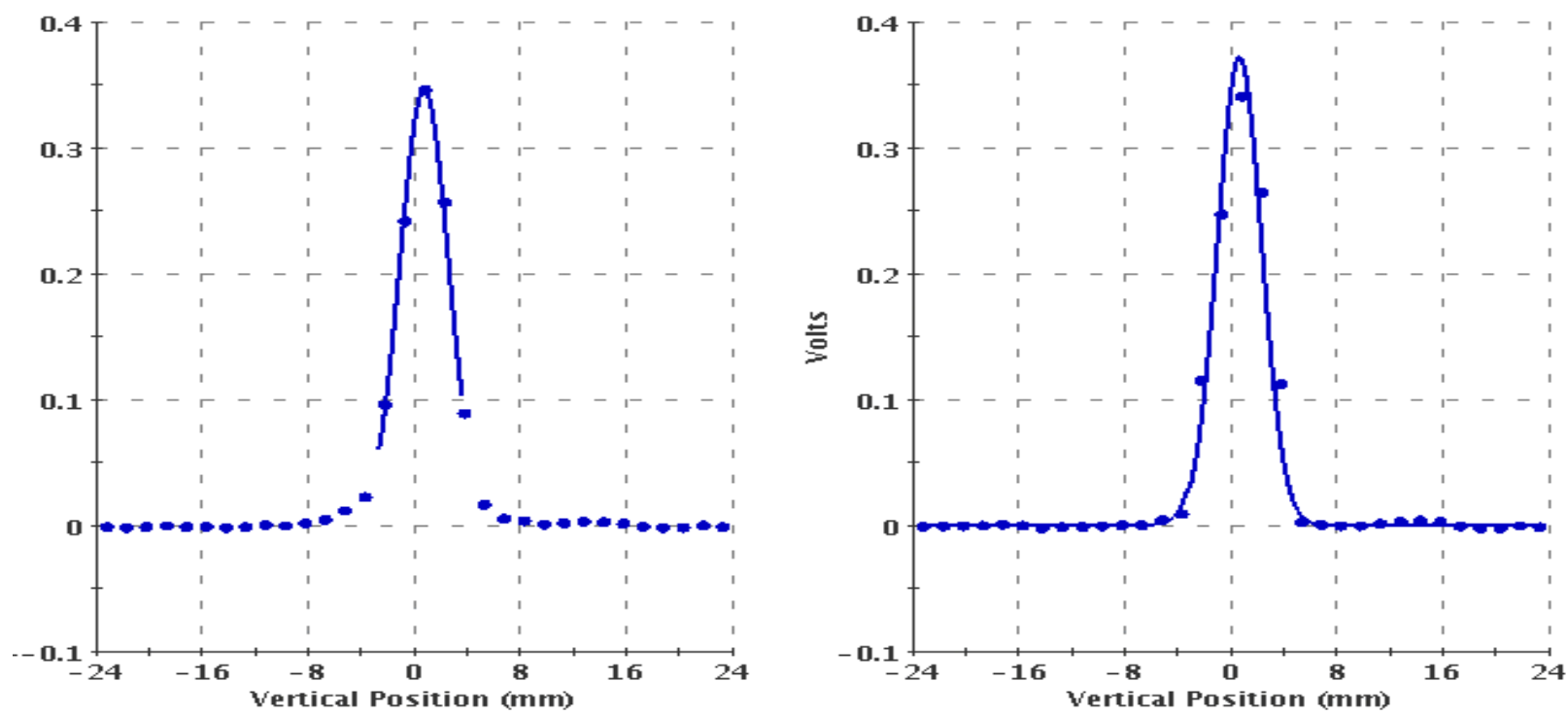

Figure 1: Comparison of BtA MW006 vertical profiles for vertical scraping at injection (left) and at the normal time later in the cycle (right).

\begin{tabular}{|l|c|c|c|c|c|}
\hline \multicolumn{1}{|c|}{ Scraping Method } & $\boldsymbol{\Delta x}$ & $\boldsymbol{\Delta} \mathbf{y}$ & $\boldsymbol{\varepsilon}_{\mathbf{x}}$ & $\boldsymbol{\varepsilon}_{\mathbf{y}}$ & $\boldsymbol{\varepsilon}_{\text {avg }}$ \\
\hline Horizontal @ injection & 3.05 & 6.35 & 7.63 & 6.35 & 6.97 \\
\hline H \& V later in cycle & 2.95 & 4.59 & 7.14 & 3.27 & 5.55 \\
\hline
\end{tabular}

Table II: Transverse normalized emittances from BtA MW006 for 2.6e11 Booster Late for horizontal scraping at injection and both horizontal and vertical scraping later in the cycle. $\Delta \mathrm{x}$ and $\Delta \mathrm{y}$ are the full widths at half maximum of a Gaussian fit in $\mathrm{mm}, \boldsymbol{\varepsilon}_{\mathrm{x}}$ and $\boldsymbol{\varepsilon}_{\mathrm{y}}$ are the normalized transverse emittances in $\pi \mathrm{mm}$ mrad, and $\varepsilon_{\mathrm{avg}}$ is the root mean square of $\varepsilon_{\mathrm{x}}$ and $\varepsilon_{\mathrm{y}}{ }^{8}$

The standard late scraping scheme was used for the remainder of the run. Although scraping at injection would be preferable because it reduces activation, it has the drawback of making it hard to notice an injection problem. Note that $\varepsilon_{\text {avg }}$ for late scraping in both planes on Jan 9 was smaller than scraping in any single plane on Dec 22 even though Booster late was substantially higher (2.6e11 vs. 2.15e11) and Booster input was lower (7.2e11 vs. 7.8e11). It also would be relevant to compare scraping in both planes at injection with scraping in both planes later in the cycle.

\footnotetext{
${ }^{6}$ Booster-AGS-PP 2017 elog Jan 92017,1857 and 1859 entries.

7 Booster-AGS-PP 2017 elog Dec 22, 20171417 and 1438 entries.

8 Booster-AGS-pp 2017 elog, Dec 222016 1356-1515 entries. The analysis in the elog uses vertical profiles that only fit their cores for the injection cases. Here I am fitting the entire vertical profile for all cases.
} 


\section{AGS Vertical Chromaticity}

The chromaticities in the AGS are typically set near zero during most of the ramp for polarization preservation. It was noticed this run that much more current was required in the vertical sextupole string to accomplish this than in previous years even though the basic setup was the same. Figure 2 shows the difference between the sextupole settings from Run 15 and those that OpticsControl calculates for both strings to make the chromaticites zero on the ramp and Figure 3 shows the same thing except for Run $17 .^{9}$

Note that in Run 17 the vertical difference gradually increases up the ramp until about $500 \mathrm{~ms}$ from At0, but in Run 15 it is more or less constant (the ramp ends around $580 \mathrm{~ms}$ ). The drop in the vertical difference after $500 \mathrm{~ms}$ likely has to do with the vertical current not being able to ramp up fast enough because of voltage limitations. By reducing both the horizontal and vertical currents the vertical power supply does not need to ramp up as fast to maintain zero vertical chromaticity. So, the vertical chromaticity is kept near zero after $500 \mathrm{~ms}$ at the expense of moving the horizontal chromaticity further from zero.

Figure 4 shows a set of chromaticity $(\xi)$ measurements made during Run 17 with the normal setup as displayed in the AGSModelViewer application. ${ }^{10}$ Note that both chromaticites (green and blue dots) are close to zero until $500 \mathrm{~ms}$ where the horizontal chromaticity becomes negative. The traces without dots are what OpticsControl calculates that the chromaticities should be using its model. The expected $\xi_{\mathrm{y}}$ is much more positive than the measured, and the expected $\xi_{\mathrm{x}}$ is more negative than the measured. The difference between the expected and measured $\xi_{\mathrm{y}}$ is greater than that for $\xi_{\mathrm{x}}$.

Figure 5 shows horizontal and vertical $\xi$ measurements without snakes or snake corrections and with no current in the sextupoles. The figure shows the measured $\xi_{\mathrm{x}}$ (blue) and $\xi_{\mathrm{y}}$ (green), the madx $\xi_{\mathrm{x}}$ (black) and $\xi_{\mathrm{y}}$ (red) values calculated through AGSModelViewer using a snapramp from that time, and the $\xi_{\mathrm{x}}$ (black solid line) and $\xi_{\mathrm{y}}$ (red solid line) from the OpticsControl model. Unlike in figure 4 , the $\xi_{\mathrm{y}}$ measurements are not far off from the

\footnotetext{
${ }^{9}$ There was a chromaticity measurement made on Jan 42015 by N. Tsoupas with very similar sextupole settings to the ones used during much of that run. At least in the interval from 360 to $600 \mathrm{~ms}$, which is the interval l'll mainly consider in this section, $\xi_{y}$ was essentially zero and $\xi_{x}$ was quite close to zero ( $\leq-1$ or -0.1 in OpticsControl units). See Booster-AGS-PP Jan 42015 elog 1248 entry and analysis in Booster-AGS-PP Sept 292017 elog 1602 and 1606 entries.

${ }^{10}$ Booster-AGS-PP 2017 elog May 23 entries from 1519 to 1833 . Tunes were measured at +2 and +7.8 $\mathrm{mm}$. The revolution frequency at At $0+400 \mathrm{~ms}$ was measured at each radius using the wall current monitor. For $+2 \mathrm{~mm}$ it was $370524.676 \mathrm{~Hz}$ and for $+7.8 \mathrm{~mm}$ it was $370513.767 \mathrm{~Hz}$ yielding a $\Delta \mathrm{f}$ of 10.909 Hz. Using $\frac{\Delta p}{p}=\frac{\gamma^{2}}{\left(\gamma_{t}^{2}-\gamma^{2}\right)} \frac{\Delta f}{f}$ with $\gamma=14.289$ and $\gamma_{\mathrm{t}}=8.47, \Delta \mathrm{p} / \mathrm{p}$ was found to be 3.26e-3. The AGSModelViewer program calculates and plots $\xi=\Delta Q /(\Delta \mathrm{p} / \mathrm{p})$ for each time in the tune scans and displays them.
} 
OpticsControl model. As for $\xi_{\mathrm{x}}$, although the difference between the measured values and the OpticsControl model in both cases is substantial, it is about the same in either case.

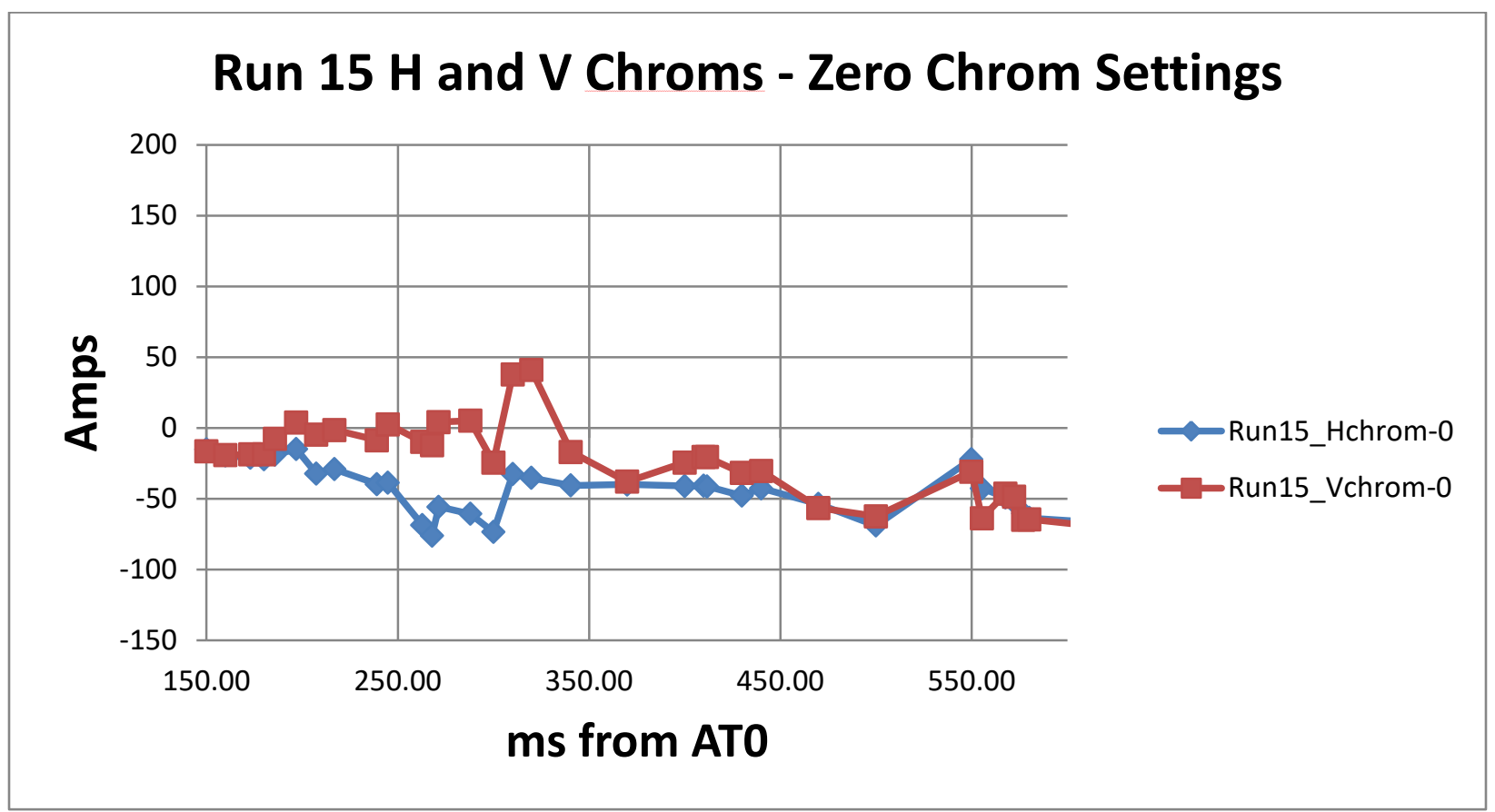

Figure 2: The difference between the sextupole settings from Run 15 and those that OpticsControl calculates for both strings to make the chromaticites zero on the ramp. Blue is the horizontal and red is the vertical string.

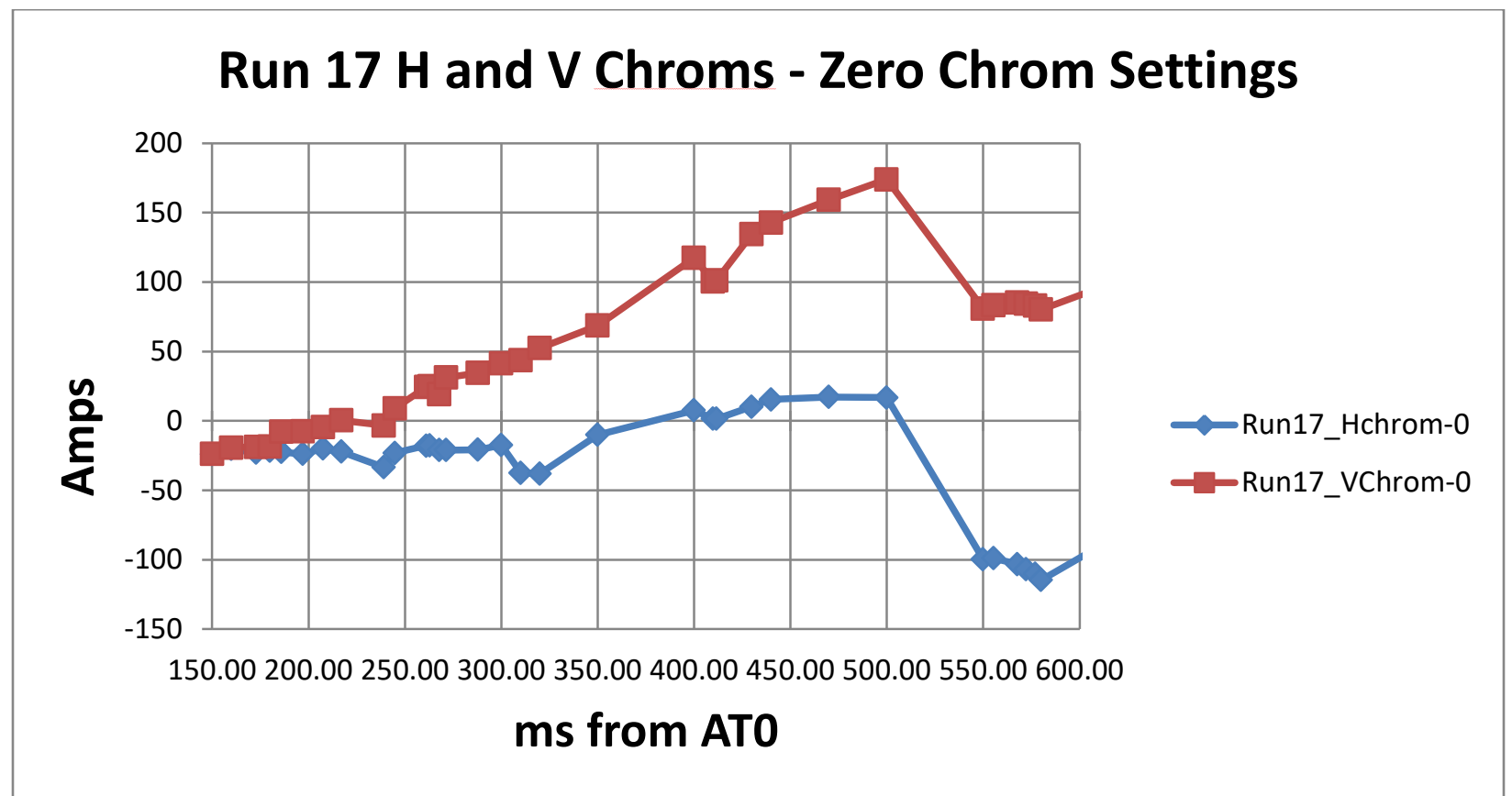

Figure 3: Same as Figure 2 except the difference is between the Run 17 sextupole settings and those that OpticsControl calculates for both strings to make the chromaticites zero on the ramp. Blue is the horizontal and red is the vertical string. 


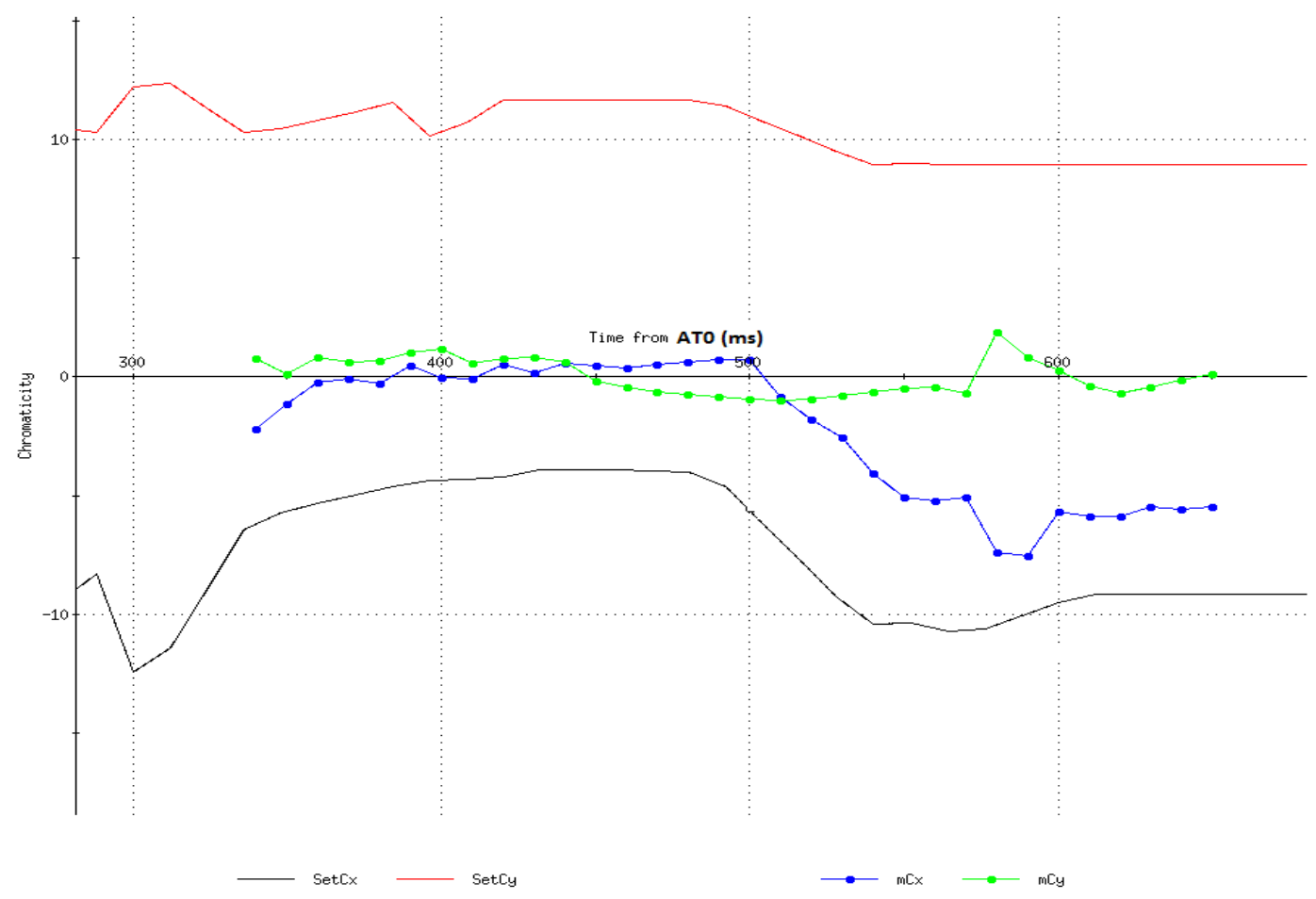

Figure 4: Measured and expected Chromaticities during the AGS ramp. This is for AU2, the nominal PP setup with snakes on. $X$-axis is time from At0, y-axis is chromaticity, $\xi=\Delta Q /(\Delta p / p)$. The red and black lines are what the OpticsControl model predicts that the vertical and horizontal chromaticities should be, respectively. The green and blue lines are the measured vertical and horizontal chromaticities respectively. The dots are the actual measurements. The display is from AGSModelViewer.

Unfortunately, OpticsControl (O.C.) uses $\xi=(\Delta \mathrm{Q} / \mathrm{Q}) /(\Delta \mathrm{p} / \mathrm{p})$ as the definition of chromaticity and AGSModelViewer uses $\xi=\Delta \mathrm{Q} /(\Delta \mathrm{p} / \mathrm{p})$. I will be switching back and forth between these definitions in the discussion below.

Chromaticity measurements were also made with the snakeless machine where the requested chromaticity in both planes was shifted one plane at a time by +1 (in OpticsControl units) from the bare $\xi$ state (see Figure 6). The change in $\xi(\Delta \xi)$ in the plane where the requested $\xi$ was shifted was about the amount expected, with $\xi$ in the other plane changing by a relatively small amount. A shift of +1 O.C. unit in the horizontal makes the vertical slightly more negative (about -0.1 O.C. unit) and a shift of +1 O.C. unit in the vertical shifts the horizontal about -0.1 in O.C. units as well.

Using $\mathrm{Q}_{\mathrm{x}}=8.78$ and $\mathrm{Q}_{\mathrm{y}}=8.81$, the change in $\xi$ in O.C. units, which I'll call $\Delta \xi^{*}$, can be approximated as $\Delta \xi / \mathrm{Q}$ where $\Delta \xi$ is the change in measured $\xi$ shown in AGSModelViewer. From the data in Figure 6, $\Delta \xi^{*}$ was calculated in each plane for 12 times in the cycle and the data is shown in Table III. 


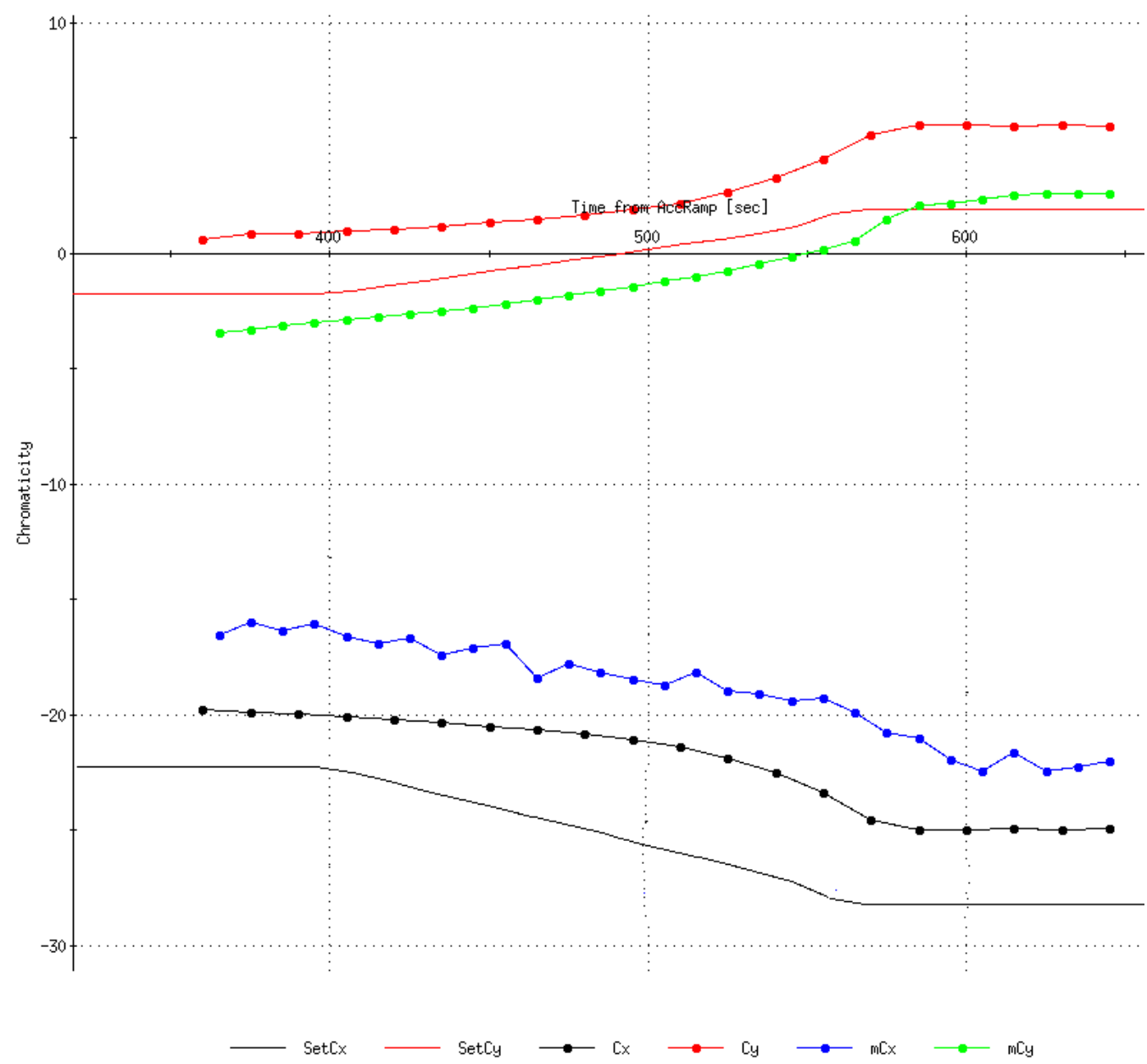

Figure 5: Measured and expected Chromaticities during the AGS ramp without snakes and with zero current in the sextupoles. The figure shows the measured $\xi_{\mathrm{x}}$ (blue) and $\xi_{\mathrm{y}}$ (green), the madx $\xi_{\mathrm{x}}$ (black) and $\xi_{\mathrm{y}}$ (red) values calculated through AGSModelViewer using a snapramp from that time, and the $\xi_{\mathrm{x}}$ (black line) and $\xi_{\mathrm{y}}$ (red line) from the OpticsControl model. ${ }^{11}$ The $\mathrm{x}$-axis is time from At0 in milliseconds and the y-axis is chromaticity.

For the snakeless machine the average of $\Delta \xi^{*}$ in each plane is the same, +0.822 instead of the expected +1 . The horizontal tune measurements are more noisy than the vertical, and so the values for $\Delta \xi_{\mathrm{x}}{ }^{*}$ bounce around more than they do for $\Delta \xi_{\mathrm{y}}{ }^{*}$. Although not as clear in the horizontal, $\Delta \xi^{*}$ gradually gets smaller up the ramp starting at a value around 0.88 at $365 \mathrm{~ms}$ and dropping to about 0.80 on the flattop (see Figure 7). The vertical $\xi$ measurements seem clean enough to detect a dip in $\Delta \xi_{\mathrm{y}}{ }^{*}$ just before the flattop (from $\sim 550$ to $580 \mathrm{~ms}$ ) which $\Delta \xi_{\mathrm{y}}{ }^{*}$ seems to recover from on the flattop. From Figure 5 the values of the bare $\xi_{\mathrm{y}}$ are not far off from the

\footnotetext{
${ }^{11}$ Booster-AGS-PP May 182017 elog, 1756 through 1952 and May 191333 to 1404 entries. Radii of +1.4 and -9 $\mathrm{mm}$ were used with $\mathrm{f}=370549.628$ and $370529.796 \mathrm{~Hz}$ giving a $\Delta \mathrm{f}$ of $19.83 \mathrm{~Hz}$ at $400 \mathrm{~ms}$ for a $\Delta \mathrm{p} / \mathrm{p}$ of $5.91 \mathrm{e}-3$ (a $\Delta p / p$ of $5.87 e-3$ was inadvertently used, but the difference is small enough to be ignored).
} 
OpticsControl model, though the bare $\xi_{\mathrm{x}}$ values are about 0.7 OpticsControl units more positive than that model.

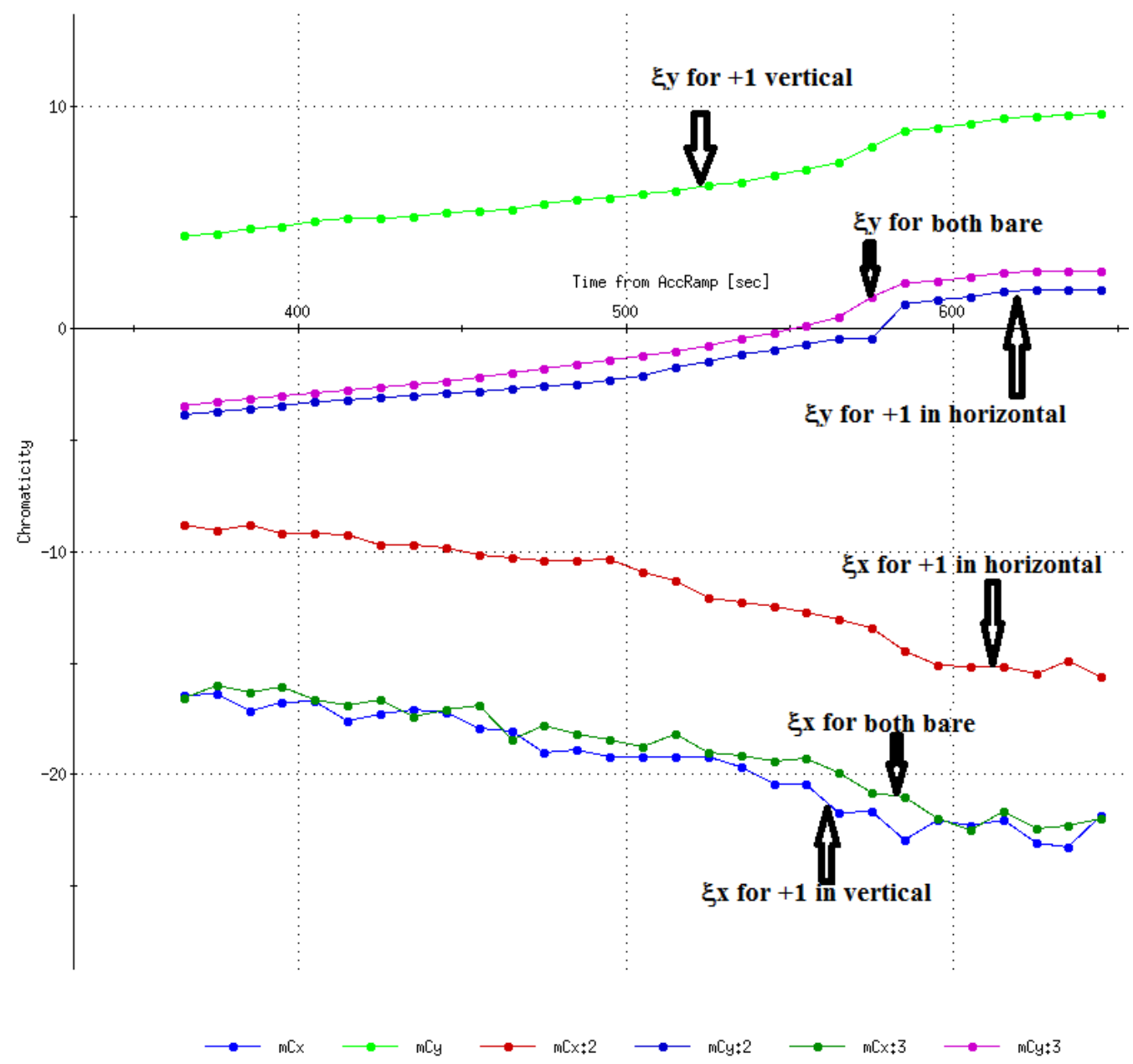

Figure 6: Chromaticity measurements on AGS ramp with no snakes and the PP magnet cycle. For bare+1 in $\mathrm{y}$ and bare in $\mathrm{x}$ : $\xi_{\mathrm{y}}$ in lighter green (upper trace), $\xi_{\mathrm{x}}$ in blue (lowest trace), for bare +1 in $\mathrm{x}$ and bare in $\mathrm{y}$ : $\xi_{\mathrm{x}}$ in red and $\xi_{\mathrm{y}}$ in blue, for bare in $\mathrm{x}$ and $\mathrm{y}: \xi_{\mathrm{x}}$ in green and $\xi_{\mathrm{y}}$ in magenta.

Assuming linear changes in $\xi$ with sextupole current it's rather straightforward to predict what currents would be required to obtain a particular $\left(\xi_{\mathrm{x}}, \xi_{\mathrm{y}}\right)$ using the data in Table III. In the equation below, $\xi_{\mathrm{x}}$ ' and $\xi_{\mathrm{y}}$ ', the chromaticites after a shift by $\left(\Delta \xi_{\mathrm{x}}{ }^{\prime}, \Delta \xi_{\mathrm{y}}{ }^{\prime}\right)$ from $\left(\xi_{\mathrm{x}}, \xi_{\mathrm{y}}\right)$, can be set to 0 and $\Delta \xi_{\mathrm{x}}{ }^{\prime}$ and $\Delta \xi_{\mathrm{y}}{ }^{\prime}$ can be solved for to find the amount of shift required to obtain zero chromaticity in both planes,

$$
\left(\begin{array}{l}
\xi^{\prime}{ }_{x} \\
\xi^{\prime}
\end{array}\right)=\left(\begin{array}{ll}
a & b \\
c & d
\end{array}\right)\left(\begin{array}{l}
\Delta \xi_{x}^{\prime} \\
\Delta \xi_{y}^{\prime}
\end{array}\right)+\left(\begin{array}{l}
\xi_{x} \\
\xi_{y}
\end{array}\right)
$$


where a, b, c, and d are the values for $\Delta \xi_{\mathrm{x}}{ }^{*}$, $\left(\Delta \xi_{\mathrm{x}}{ }^{*}\right)$ for +1 in $\xi_{\mathrm{y}}, \Delta \xi_{\mathrm{y}}{ }^{*}$, and $\left(\Delta \xi_{\mathrm{y}}{ }^{*}\right)$ for +1 in $\xi_{\mathrm{x}}$ respectively. Table IV shows $\left(\Delta \xi_{\mathrm{x}}{ }^{\prime}, \Delta \xi_{\mathrm{y}}{ }^{\prime}\right)$ for the times in Table III and Figure 8 shows what the set chromaticities look like in OpticsControl when these shifts are applied.

\begin{tabular}{|l|l|l|l|l|l|l|l|l|}
\hline $\begin{array}{l}\text { Time } \\
(\mathrm{ms})\end{array}$ & $\xi_{\mathrm{x}}$ for bare & $\xi_{\mathrm{x}}$ for +1 in $\mathrm{x}$ & $\xi_{\mathrm{y}}$ for bare & $\xi_{\mathrm{y}}$ for +1 in $\mathrm{y}$ & $\Delta \xi_{\mathrm{x}}{ }^{*}$ & $\Delta \xi_{\mathrm{y}}{ }^{*}$ & $\begin{array}{l}\left(\Delta \xi_{\mathrm{x}}{ }^{*} \text { ) for }\right. \\
+1 \text { in } \xi_{\mathrm{y}}\end{array}$ & $\begin{array}{l}\left(\Delta \xi_{\mathrm{y}}{ }^{*}\right) \text { for } \\
+1 \text { in } \xi_{\mathrm{x}}\end{array}$ \\
\hline 365 & -16.62 & -8.77 & -3.38 & 4.35 & 0.894 & 0.877 & 0 & -0.044 \\
\hline 400 & -16.36 & -9.09 & -2.79 & 4.87 & 0.828 & 0.869 & -0.044 & -0.051 \\
\hline 411 & -16.75 & -9.22 & -2.66 & 4.94 & 0.858 & 0.863 & -0.059 & -0.044 \\
\hline 450 & -17.01 & -9.87 & -2.08 & 5.45 & 0.813 & 0.855 & -0.067 & -0.066 \\
\hline 475 & -17.86 & -10.39 & -1.69 & 5.78 & 0.851 & 0.848 & -0.141 & -0.081 \\
\hline 500 & -18.64 & -10.58 & -1.17 & 6.17 & 0.918 & 0.833 & -0.067 & -0.096 \\
\hline 525 & -19.16 & -12.08 & -0.65 & 6.69 & 0.806 & 0.833 & -0.007 & -0.081 \\
\hline 555 & -19.29 & -12.72 & 0.26 & 7.34 & 0.747 & 0.804 & -0.126 & -0.096 \\
\hline 570 & -20.39 & -13.25 & 1.17 & 8.05 & 0.813 & 0.781 & -0.148 & -0.169 \\
\hline 578 & -20.71 & -13.70 & 1.82 & 8.364 & 0.798 & 0.774 & -0.133 & -0.155 \\
\hline 600 & -22.27 & -15.13 & 2.40 & 9.35 & 0.813 & 0.789 & 0 & -0.103 \\
\hline 626 & -22.53 & -15.52 & 2.73 & 9.74 & 0.798 & 0.796 & -0.074 & -0.088 \\
\hline Avg. & & & & & 0.822 & 0.822 & -0.094 & -0.079 \\
\hline
\end{tabular}

Table III: Measured changes in $\xi$ for a +1 OpticsControl unit change in requested $\xi$ from bare $\xi$ in either plane. $\Delta \xi_{x}{ }^{*}$ and $\Delta \xi_{y}{ }^{*}$ are the measured changes in OpticsControl units. " $\left(\Delta \xi_{x}{ }^{*}\right)$ for +1 in $\xi_{y}$ " is the change in $\Delta \xi_{\mathrm{x}}$ due to a +1 unit change in $\xi_{\mathrm{y}}$ and similarly " $\left(\Delta \xi_{\mathrm{y}}{ }^{*}\right)$ for +1 in $\xi_{\mathrm{x}}$ " is the change in $\Delta \xi_{\mathrm{y}}$ due to a +1 unit change in $\xi_{x}$. The data is taken from Figure 6 and converted to OpticsControl units as described in the text.

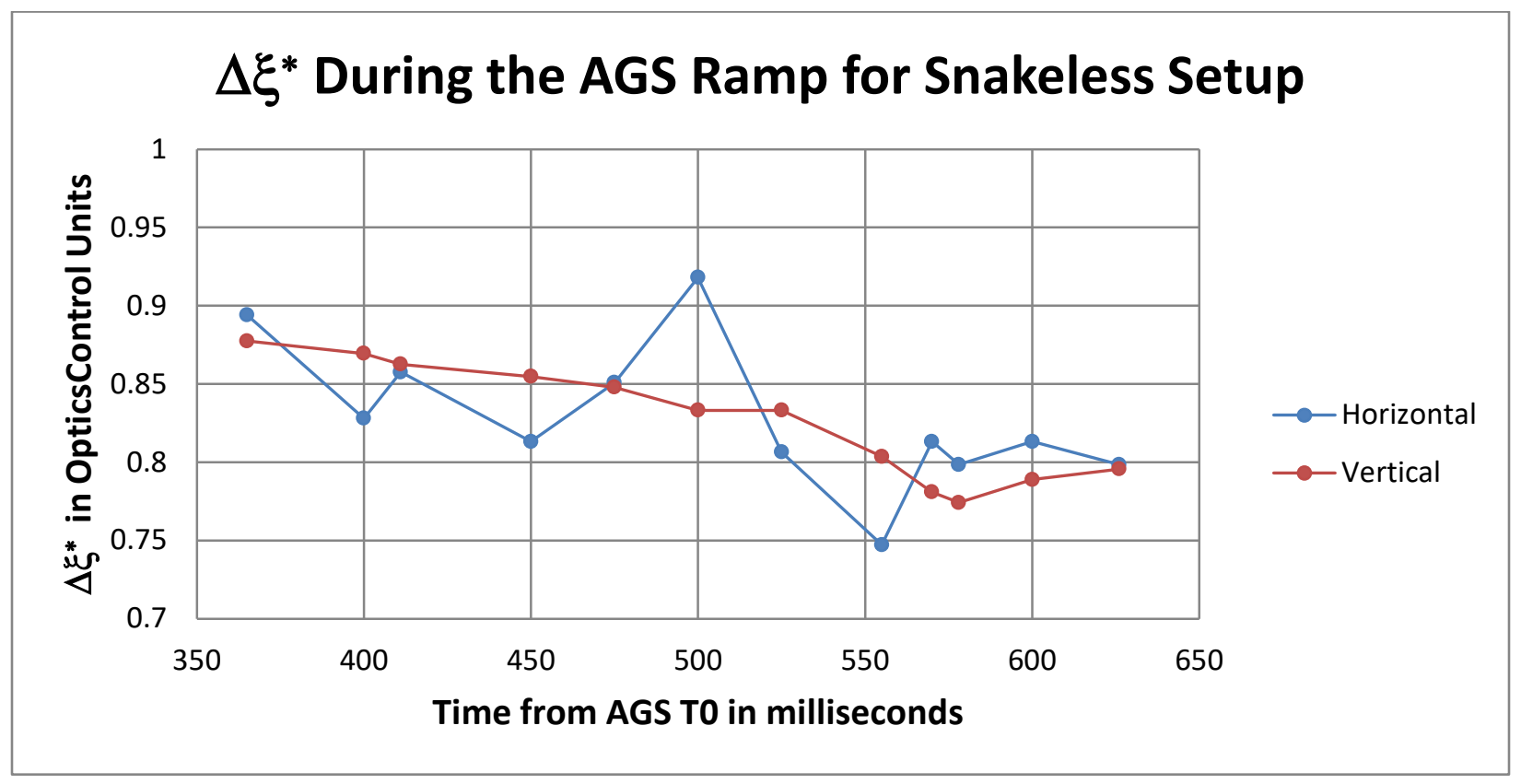

Figure 7: A plot of the data in Table III for $\Delta \xi_{\mathrm{x}}{ }^{*}$ and $\Delta \xi_{\mathrm{y}}{ }^{*}$ at different times in the AGS cycle. 


\begin{tabular}{|l|l|l|}
\hline Time (ms) & $\Delta \xi_{\mathrm{x}}{ }^{\prime}$ & $\Delta \boldsymbol{\xi}_{\mathrm{y}}{ }^{\prime}$ \\
\hline 365 & 0.544 & 2.117 \\
\hline 400 & 0.499 & 2.277 \\
\hline 411 & 0.466 & 2.420 \\
\hline 450 & 0.464 & 2.420 \\
\hline 475 & 0.462 & 2.467 \\
\hline 500 & 0.429 & 2.344 \\
\hline 525 & 0.352 & 2.709 \\
\hline 555 & 0.320 & 2.994 \\
\hline 570 & 0.467 & 2.941 \\
\hline 578 & 0.335 & 3.010 \\
\hline 600 & 0.063 & 3.119 \\
\hline 626 & -0.035 & 3.211 \\
\hline
\end{tabular}

Table IV: Predicted shifts in $\xi_{\mathrm{x}}$ and $\xi_{\mathrm{y}}$ from bare $\xi$ for zero $\xi$ in both planes using equation (1). These shifts are in O.C. units.

In the normal Run 17 running state, before $500 \mathrm{~ms}$ when both chromaticites are near zero, the requested $\xi_{\mathrm{y}}$ is about +1.3 units and the requested $\xi_{\mathrm{x}}$ is about -0.5 units. In Run 15 the set $\xi_{\mathrm{y}}$ during this interval was very close to zero, and the set $\xi_{\mathrm{x}}$ was around -0.5 . The predicted zero chromaticities for the snakeless state are roughly +0.35 in the vertical and -0.3 in the horizontal. The predicted $\xi_{\mathrm{x}}$ is not that different than either of the typical Run 15 or 17 cases, but the predicted $\xi_{\mathrm{y}}$ is quite different from either of those. It is higher than the Run 15 case and much lower than the Run 17 case. Figure 9 shows the sextupole currents for the predicted case as well as for typical configurations during Run 15 and 17 . Whether or not these predicted currents actually make both chromaticities come close to zero was not checked.

\section{Measuring the Response of Individual Sextupole Magnets}

To determine if there is a problem with a particular sextupole magnet, the effect of each of the horizontal and vertical sextupoles on the tune was measured with the snakes off at injection energy. +50A was put into one string and a local horizontal bump was introduced at the location of a magnet powered by that string. The relevant tune was measured, then the bump's polarity was reversed, the tune was measured again, and the difference between the 2 tune values was found $(\Delta \mathrm{Q})$. This was done for each sextupole location. Figure 10 shows the results. ${ }^{12}$

In all cases the sign of the tune change was as expected, indicating that the polarity of each of the sextupoles is likely correct. $\Delta \mathrm{Q}$ was generally larger for horizontal than for vertical magnets. For the horizontal sextupoles, the average $\Delta \mathrm{Q}_{\mathrm{x}}$ was 0.034 and for the vertical ones the average $\Delta \mathrm{Q}_{\mathrm{y}}$ was 0.024 . The amplitude of the vertical bumps was generally not smaller than for the horizontal ones so it's not clear why $\Delta \mathrm{Q}_{\mathrm{y}}$ seems to be larger for the horizontal string than for the vertical.

\footnotetext{
${ }^{12}$ See June 272017 Booster-AGS-PP elog 1643 entry and Jun 281608 entry.
} 


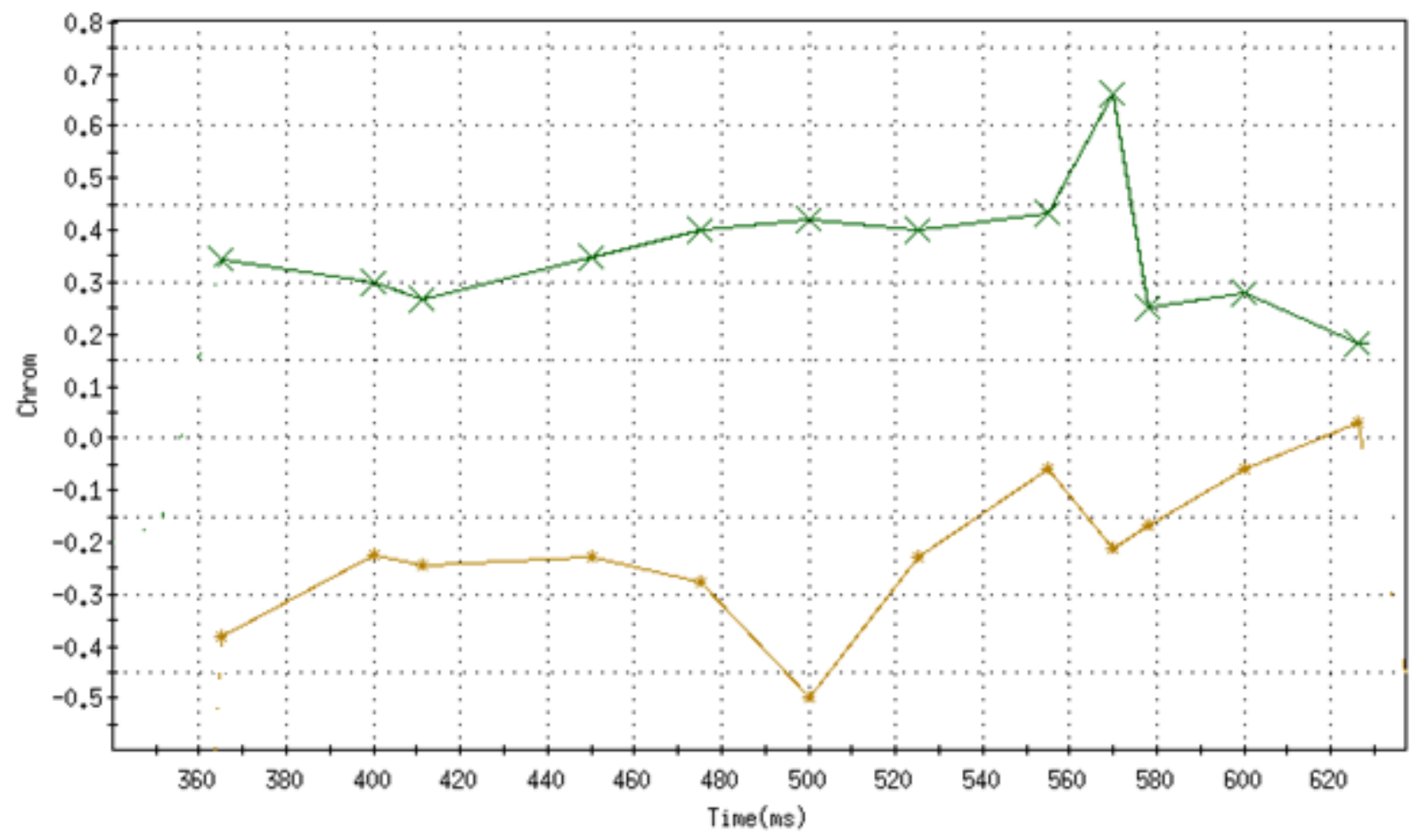

Figure 8: A display from OpticsControl showing the predicted settings, extrapolated from the measured chromaticities described above for the snakeless machine, which would make both chromaticites zero at the points shown. Green is $\xi_{\mathrm{y}}$ and brown is $\xi_{\mathrm{x}}$. The beginning of the flattop is near $580 \mathrm{~ms}$.

\section{Other Observations}

With the snakes off and at injection energy if the chromaticities are set to 0 , the coherence is long in both planes. With $\xi_{\mathrm{x}}$ set to zero and $\xi_{\mathrm{y}}$ set to +3.0 O.C. units, the horizontal chromaticity is still close to zero, and the measured $\xi_{\mathrm{y}}$ is +1.5 , here $\left(\mathrm{I}_{\mathrm{x}}, \mathrm{I}_{\mathrm{y}}\right)=(50 \mathrm{~A}, 75 \mathrm{~A})$. With these $\xi$ settings, the time it takes the beam to decohere is longest when the radius is close to zero (see Table V). ${ }^{13}$

Roughly the same radial vertical coherence dependence exists when either of the 2 strings is powered separately (there's no data available for the horizontal), but with zero current in both strings there is no obvious radial dependence in the vertical decoherence time suggesting the dependence is related to current in the sextupoles. The radial dependence suggests that the actual chromaticites may depend on the radius. So, if the radius was different in Run 15 than 17 than different currents in the sextupoles might be required to reach zero chromaticity. The radius on the ramp was changed during Run 17 on Jan 18 from $-2 \mathrm{~mm}$ to $+2 \mathrm{~mm}$, but the need for more vertical sextupole current existed before that change, when the radius was about the same as in

${ }^{13}$ See June 282017 Booster-AGS-PP elog 
Run 15. It was also noticed (in the PP setup) that the coherence doesn't last as long when the strength of the tunemeter kick is increased. ${ }^{14}$
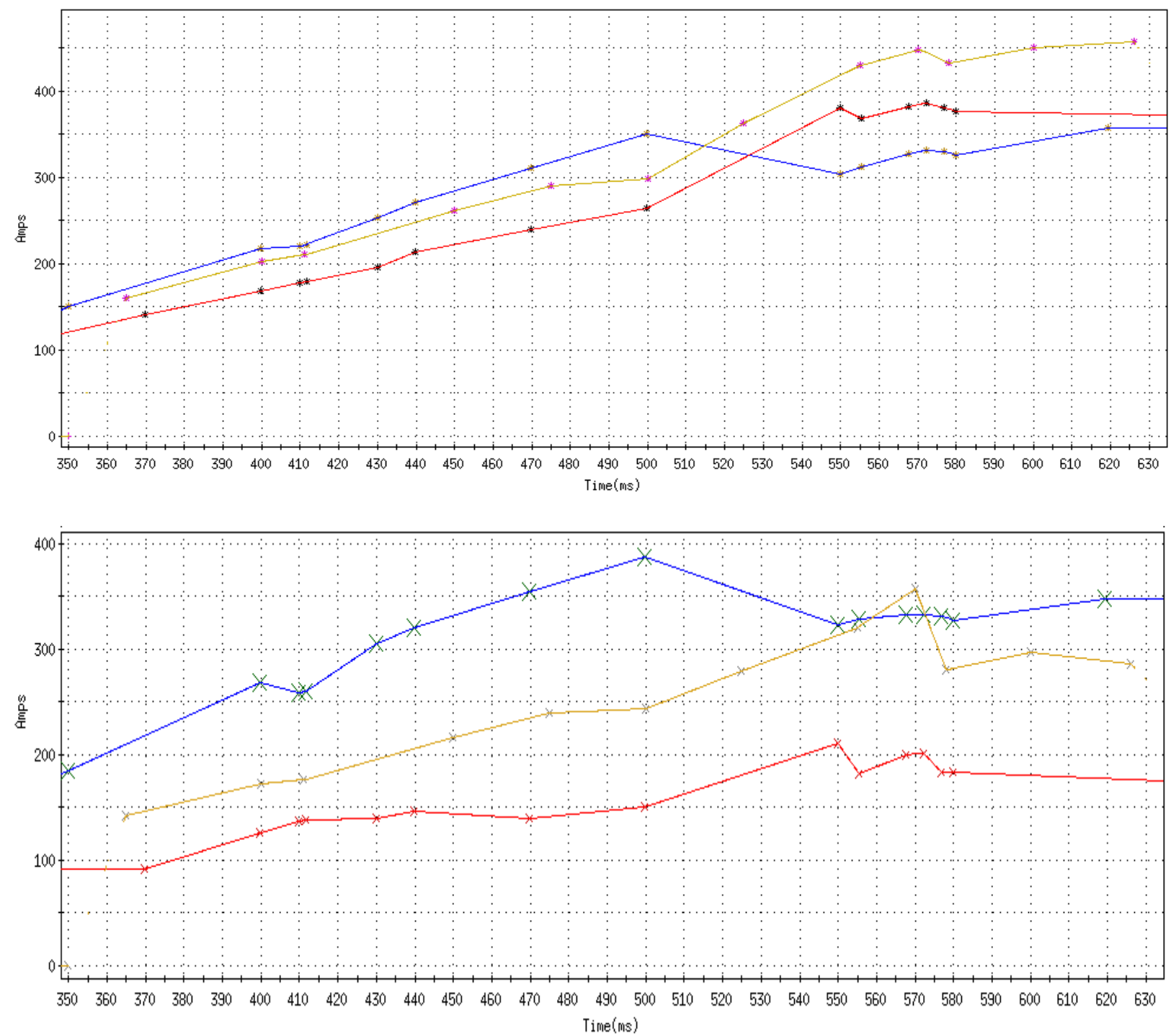

Figure 9: Sextupole currents for Run 17 (blue), Run 15 (red), and predicted in snakeless machine (gold). The top plot is the horizontal and the bottom plot is the vertical. Time is from At $0 .{ }^{15}$ Note that for the Run 17 PP setup the measured $\xi_{\mathrm{x}}$ is not near zero after $500 \mathrm{~ms}$.

\footnotetext{
${ }^{14}$ See Booster AGS PP Feb 12017 elog 1816 entries

${ }^{15}$ Run 15 data is taken from the May 1820150404 user4 archive and Run 17 data is from the May 1820170202 user 2 archive.
} 


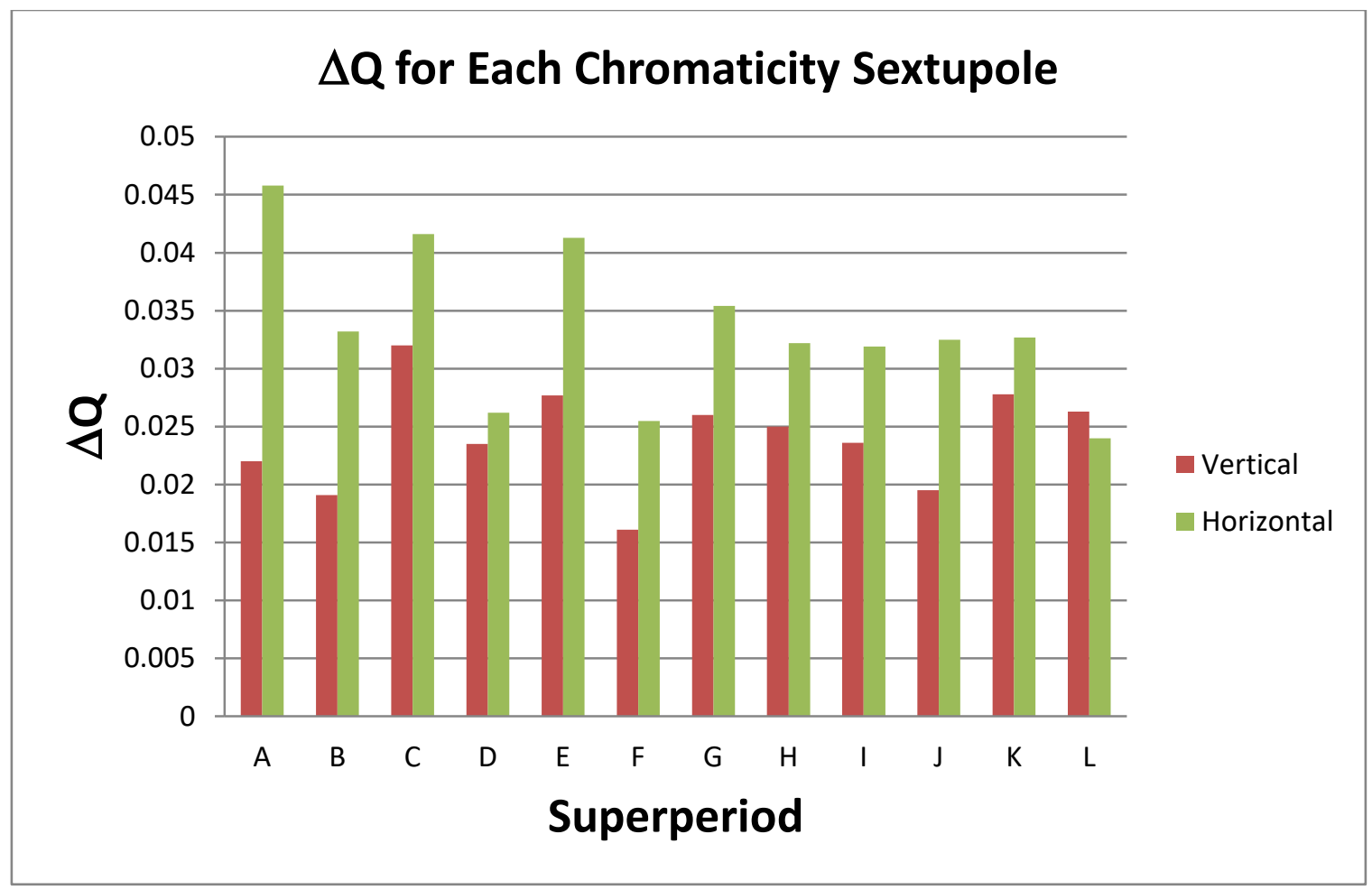

Figure 10: The measured tune change $(\Delta \mathrm{Q})$ between a positive and a negative local bump at the location of each sextupole in the snakeless machine at injection energy with $+50 \mathrm{~A}$ in either the horizontal or vertical sextupole string. In the case where a vertical sextupole is tested (red), $\Delta \mathrm{Q}$ is the change in $\mathrm{Q}_{\mathrm{y}}$, and for a horizontal sextupole (green) it is the change in $\mathrm{Q}_{\mathrm{x}}$.

\begin{tabular}{|l|l|l|}
\hline Radius $(\mathrm{mm})$ & $X$ decoherence time (turns) & $Y$ decoherence time (turns) \\
\hline-2.0 & 950 & 65 \\
\hline-0.4 & $>6000$ & 525 \\
\hline+0.4 & 6000 & 525 \\
\hline+4.8 & 250 & 270 \\
\hline
\end{tabular}

Table V; Decoherence time (in turns) as a function of measured radius at injection energy without snakes and with sextupole currents of $\left(I_{x}, I_{y}\right)=(50,75)$.

\section{The Dual Harmonic Setup in the AGS}

In Run 15 the AGS Rf harmonic was 8 , this year $\mathrm{h}=6$ was used primarily because it allows the option of running 2 cavities early in the cycle on $\mathrm{h}=12$ and phasing them such that they flatten out the vector sum of all the cavities in the neighborhood of the synchronous phase. This in turn will flatten out the bunch, and is analogous to what was done in Run 15 in the Booster using $\mathrm{h}=1$ and 2 . The situation in the AGS is more complicated though because the magnet cycle uses nearly the highest $\mathrm{dB} / \mathrm{dt}$ available for polarization preservation reasons. Normally, at a minimum, $8 \mathrm{Rf}$ cavities at full voltage are required to accelerate the beam through where $\mathrm{dB} / \mathrm{dt}$ reaches its peak without beam loss (when the bucket area is at a minimum). Figure 
11 shows the AGS magnet cycle, where injection occurs at $144 \mathrm{~ms}$ and the peak $\mathrm{dB} / \mathrm{dt}$ is attained at $268 \mathrm{~ms}$.

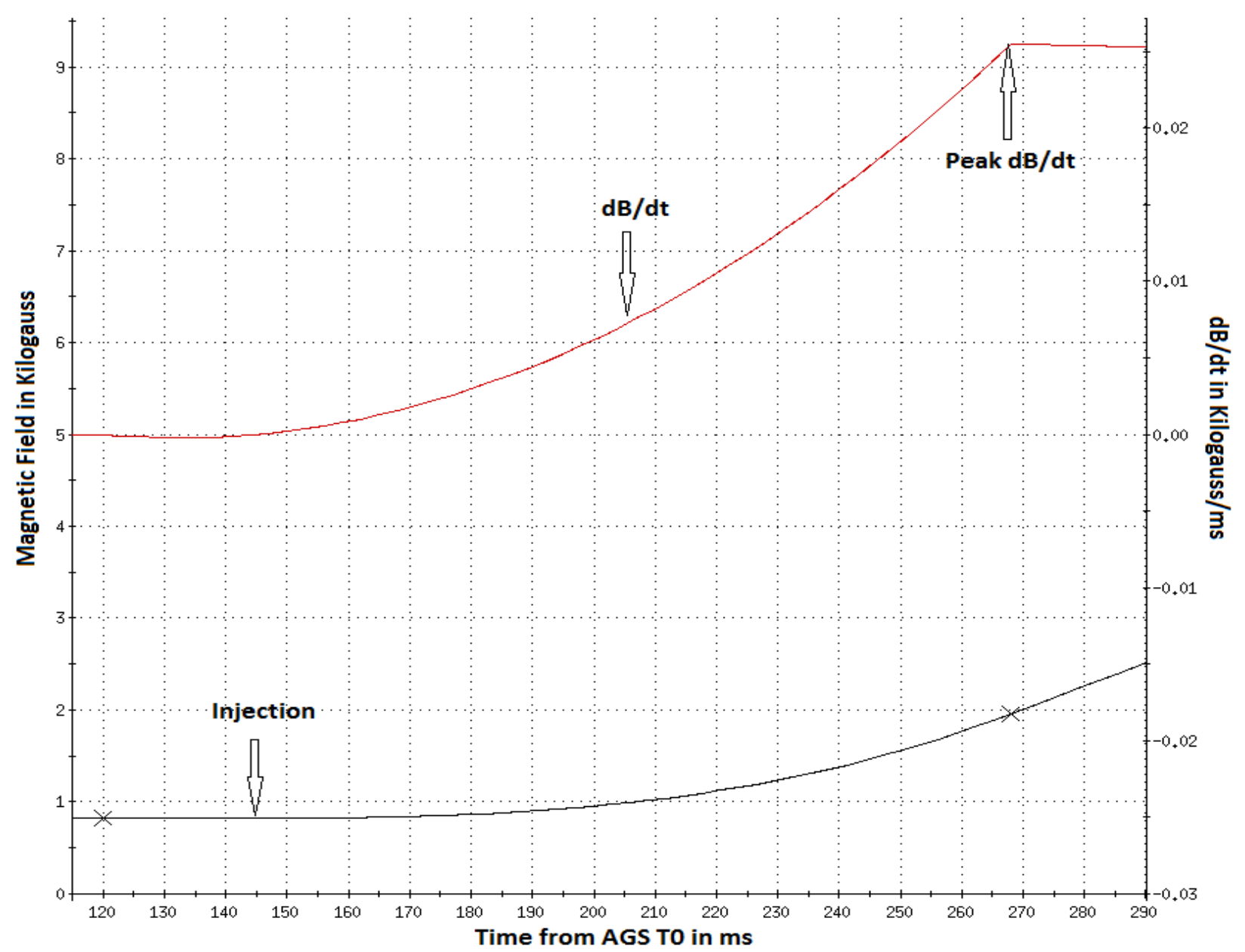

Figure 11: The early part of polarized proton AGS Main Magnet ramp. The B field (in black) is associated with the left $\mathrm{y}$-axis and $\mathrm{dB} / \mathrm{dt}$ (in red) is associated with the right $\mathrm{y}$-axis.

The first tests of this dual harmonic setup were performed in Run 16 with 9 cavities available. Using only one Rf cavity set to $\mathrm{h}=12$ had little benefit ${ }^{16}$, but 2 cavities flattened out the bunch well from injection up until about $245 \mathrm{~ms} .{ }^{17}$ After that, with the dual harmonic active the bunch became asymmetric and was lost shortly thereafter. The dual harmonic only works if the synchronous phase is below some value $\left(\sim 40^{\circ}\right)$, when it is higher than that the accelerating bucket is smaller than it would be otherwise. ${ }^{18}$

In Run 16 Rf station KL had been configured for Gold operation and was not available. For Run 17 KL was configured for protons, which made $10 \mathrm{Rf}$ stations available, though one of

\footnotetext{
${ }^{16}$ See Booster-AGS-PP 2016 elog May 21846 entry

17 See Booster-AGS-PP 2016 elog April 282106 entry

${ }^{18}$ See C. Gardner, C-A Note 535, "Booster Double Harmonic Setup Notes", February 2015, Figure 22 on page 36.
} 
the 10 could only run at about half the maximum voltage. In order to use the dual harmonic early in the cycle, and still be able to accelerate the beam properly and without beam loss, it was necessary to zero the voltage on the $2 \mathrm{~h}=12$ cavities after they were used to flatten the bunch, switch them to $h=6$, and raise their voltages back up by the time they were needed for additional accelerating voltage. This largely constrained how far into the cycle the dual harmonic could be used.

The normal RHIC filling mode used the dual harmonic setup in the AGS from March 10 to the end of the Run. In that configuration the dual harmonic stayed on up until $230 \mathrm{~ms}$ or so, then the 2 cavities used for it were lowered to zero, switched to $h=6$, and were up at sufficient voltage in time to prevent significant beam loss associated with a lack of accelerating voltage. Figure 12 shows the dual harmonic Rf voltage setup as it looked on March $10{ }^{19}$

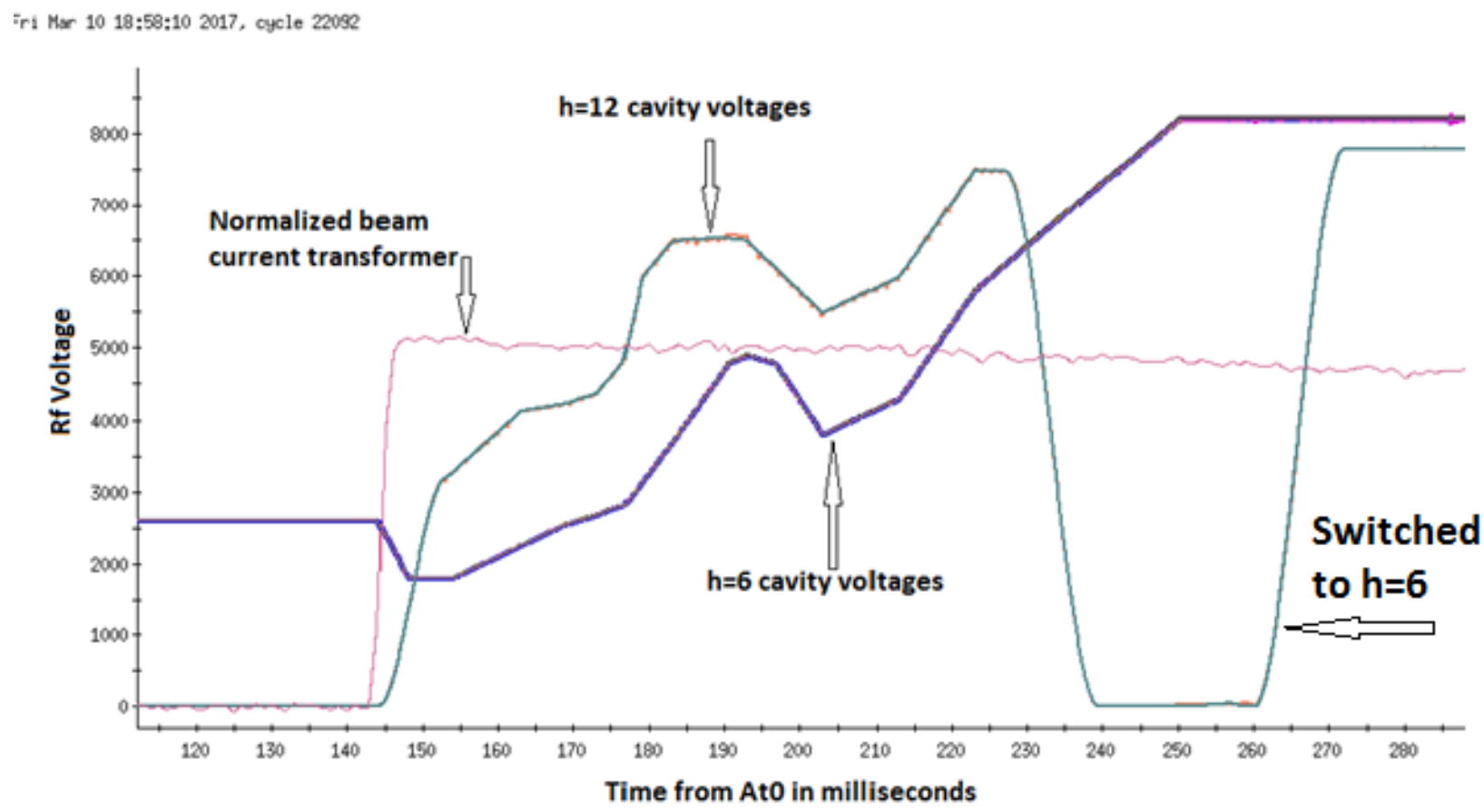

Figure 12: Rf voltages used for the dual harmonic setup in AGS. Stations B, BC, C, D, DE, E, IJ, and KL are set to $\mathrm{h}=6$ and stations $\mathrm{JK}$ and $\mathrm{K}$ are set to $\mathrm{h}=12$ for the dual harmonic than switched to $\mathrm{h}=6$. In this case, all the $\mathrm{h}=6$ cavities have the same function except for station $\mathrm{E}$ which has a lower voltage (and is not shown here). Both JK and $\mathrm{K}(\mathrm{h}=12)$ have the same voltage here. The AGS normalized beam current transformer is also shown in pink (and in arbitrary units). The y-axis is the Rf voltage in $\mathrm{kV} / \mathrm{gap}$.

Although it's not obvious in the plot, there were typically more losses from 220 to 260 ms with the dual harmonic setup. In particular, for the first month or so of running with the dual harmonic there was a rather sharp loss around 220 ms that was not present with the normal setup. Since this setup seemed to provide higher polarization the loss was not removed initially, but

${ }^{19}$ Booster-AGS-PP 2017 elog March 101853 entry 
eventually was by changing the horizontal tune slightly there. It was likely occurring at one of the tune jumps. Figure 13 shows the current transformer before and after that change.

hu Mar $3022 \div 15 \div 482017$, cycle 42305

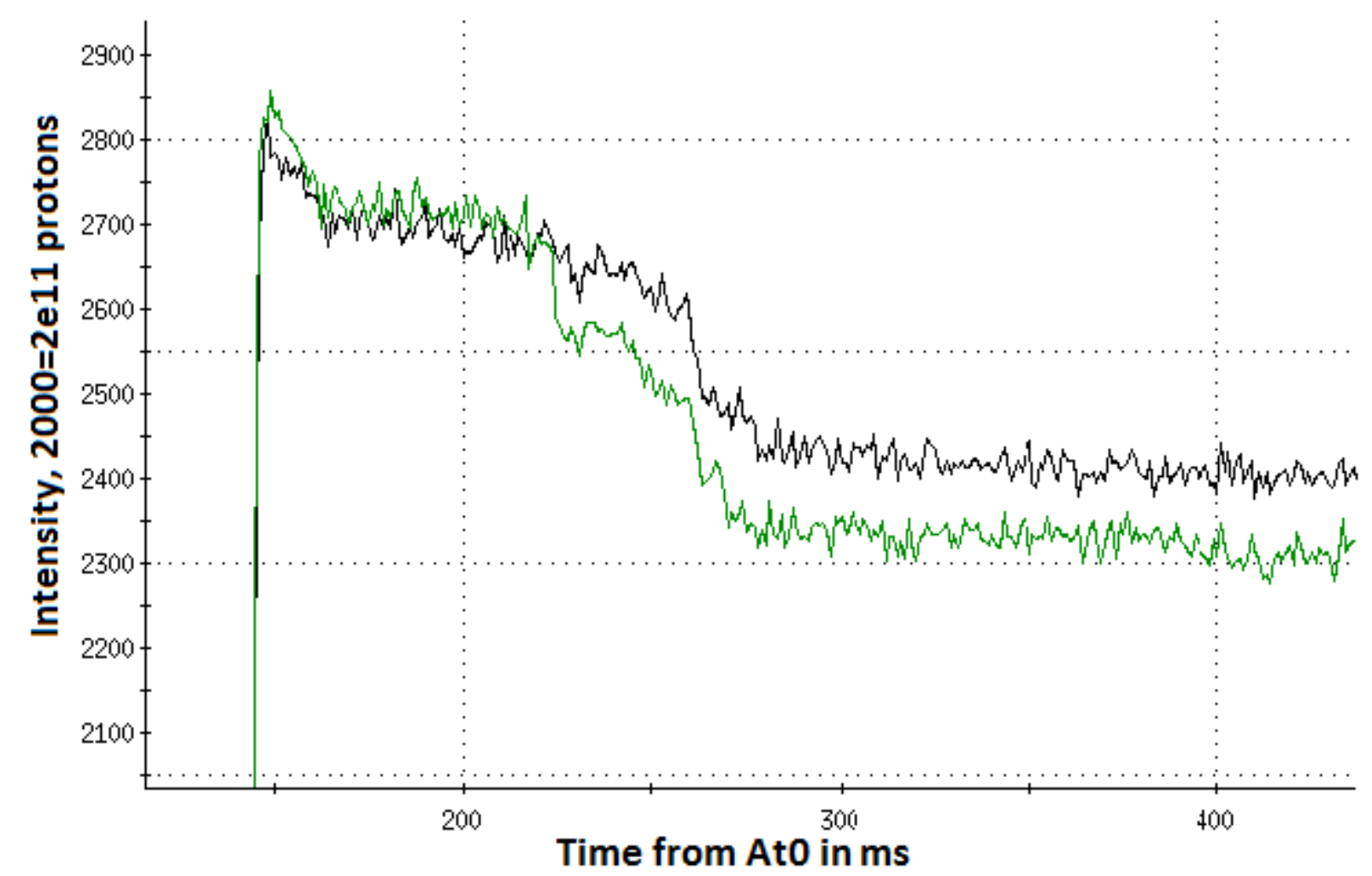

Figure 13: The AGS normalized current transformer before (green) and after (black) the change in $\mathrm{Q}_{\mathrm{x}}$ around $220 \mathrm{~ms}^{20}$

The sharp loss around $260 \mathrm{~ms}$ is actually not due to a lack of $\mathrm{Rf}$ voltage but is associated with the $0+\mathrm{Q}_{\mathrm{y}}=\mathrm{G} \gamma$ spin resonance. There is some loss (maybe 1-2\%) between 220 and $260 \mathrm{~ms}$ and perhaps a little after the resonance that may have to do with the lower Rf voltage there, but some of that loss also has to do with factors unrelated to the dual harmonic setup.

Figure 14 shows a wall current monitor (WCM) mountain range display looking at the interval from 174 to $184 \mathrm{~ms}$. On the left is a bunch with the dual harmonic on and on the right a bunch without it. In the case where it is not being used the voltage is set as low as possible to reduce the peak charge density, but in the case of the dual harmonic it's evident that the peak charge density is lower than that. The bunch is also somewhat wider, at $174 \mathrm{~ms}$ they are about 160 ns vs. 130 ns or so.

Theoretically, for the dual harmonic to work properly the $\mathrm{h}=12$ voltage should be $180^{\circ}$ out of phase with the synchronous phase. If an $\mathrm{h}=6 \mathrm{Rf}$ cavity voltage needs to be lowered for some reason, or if an $\mathrm{h}=6$ cavity is off (even if there is enough voltage to accelerate) the relative

${ }^{20}$ Booster-AGS-PP Mar 302017 elog 2214 entry 
phasing will need to be adjusted throughout the time the dual harmonic is on. During last run this was done by looking at the mountain range and adjusting the phase reference for both $\mathrm{h}=6$ cavities to flatten the bunch as much as possible. One possible future improvement to the setup would be to have the $h=12$ phase locked to the synchronous phase with a constant offset of $180^{\circ}$.

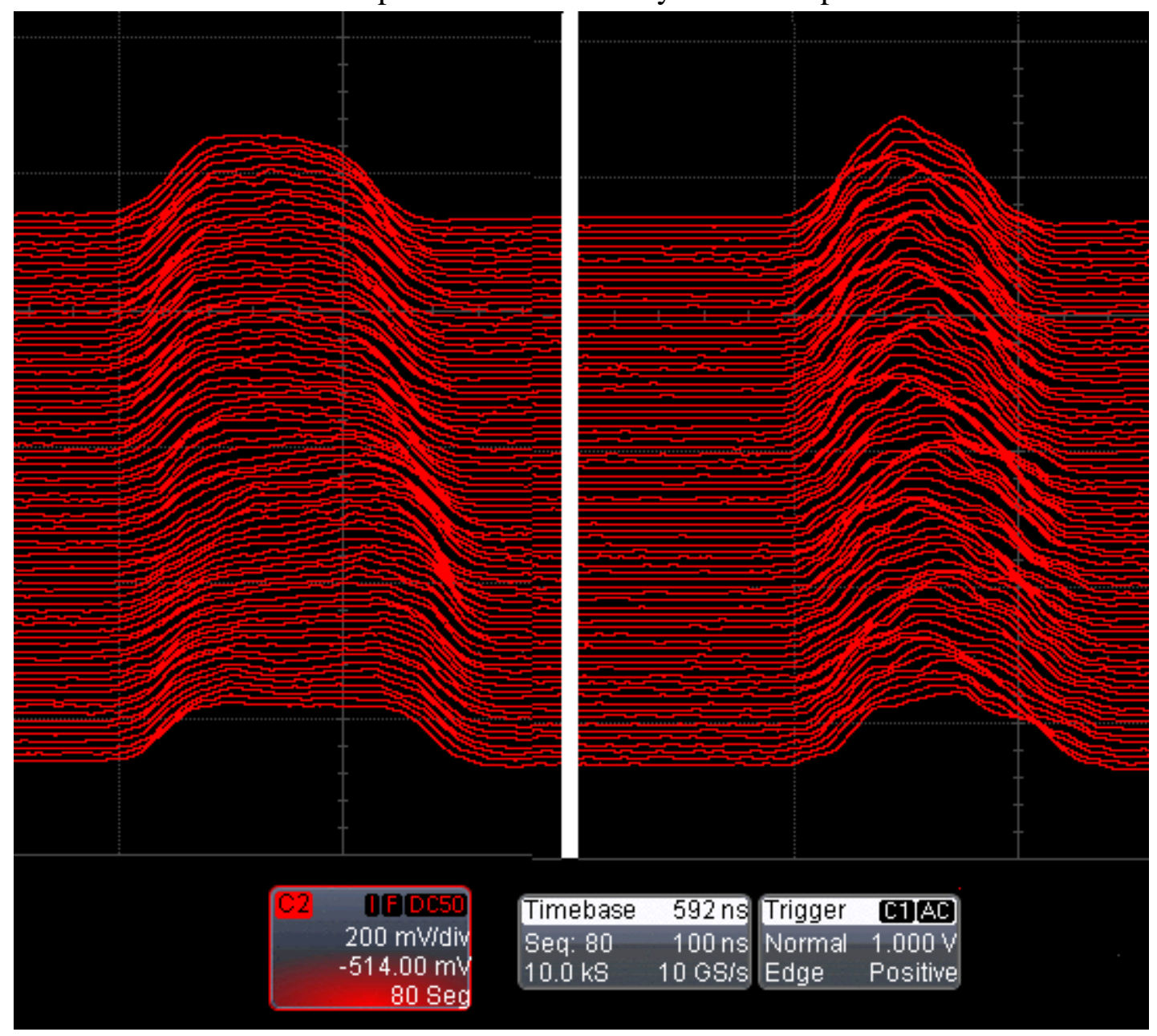

Figure 14: Wall current monitor mountain ranges for a bunch with (left) and without (right) the dual harmonic. The display is looking over the interval from 174 to $184 \mathrm{~ms}$ from At0. ${ }^{21}$

The same situation exists in the Booster, but the Booster setup is less prone to this problem because there are fewer cavities that may need adjustment ( 2 instead of 8 ). In the AGS there is also a related complication that arises at the main magnet voltage bank transfer from the F bank (used for low $\mathrm{dB} / \mathrm{dt}$ ) and the $\mathrm{P}$ bank (used for high $\mathrm{dB} / \mathrm{dt}$ ). The whole process occurs between about 188 and $194 \mathrm{~ms}$. There is a spike in the $\mathrm{dB} / \mathrm{dt}$ during that time, and more over the details of the transfer and therefore $\mathrm{dB} / \mathrm{dt}$ are not reproducible from one cycle to the next.

Figure 15 shows a magnet voltage signal in red over the first $100 \mathrm{~ms}$ of the ramp. The jump at about $45 \mathrm{~ms}$ into the ramp is the $\mathrm{F}$ to $\mathrm{P}$ transfer. First it jumps up and then it goes lower

\footnotetext{
${ }^{21}$ Booster-AGS-PP 2017 elog entries on Mar 2 at 2112 and 2135.
} 
than where it was. The blue trace is Rf Station K voltage $(\mathrm{h}=12)$. All the Rf stations need to be raised during this time. The 2 upper traces are the bunch envelope from the WCM. The purple trace is the WCM with the dual harmonic on, behind it (in yellow) is that signal without the dual harmonic.

For the most part the peak current in the dual harmonic case is substantially less but around the time of the $\mathrm{F}$ to $\mathrm{P}$ transfer the Rf voltage is increased in order to cope with it. There are also some quadrupole oscillations that are induced there. Again, the details of this behavior change from cycle to cycle. Note also that there is more magnet voltage ripple after the transfer than before it. ${ }^{22}$ This is expected for the higher $\mathrm{dB} / \mathrm{dt}$ voltage bank, but it may also make it harder to keep the $\mathrm{h}=12$ phase correct. Locking the $\mathrm{h}=12$ to the synchronous phase might also help in this regard.

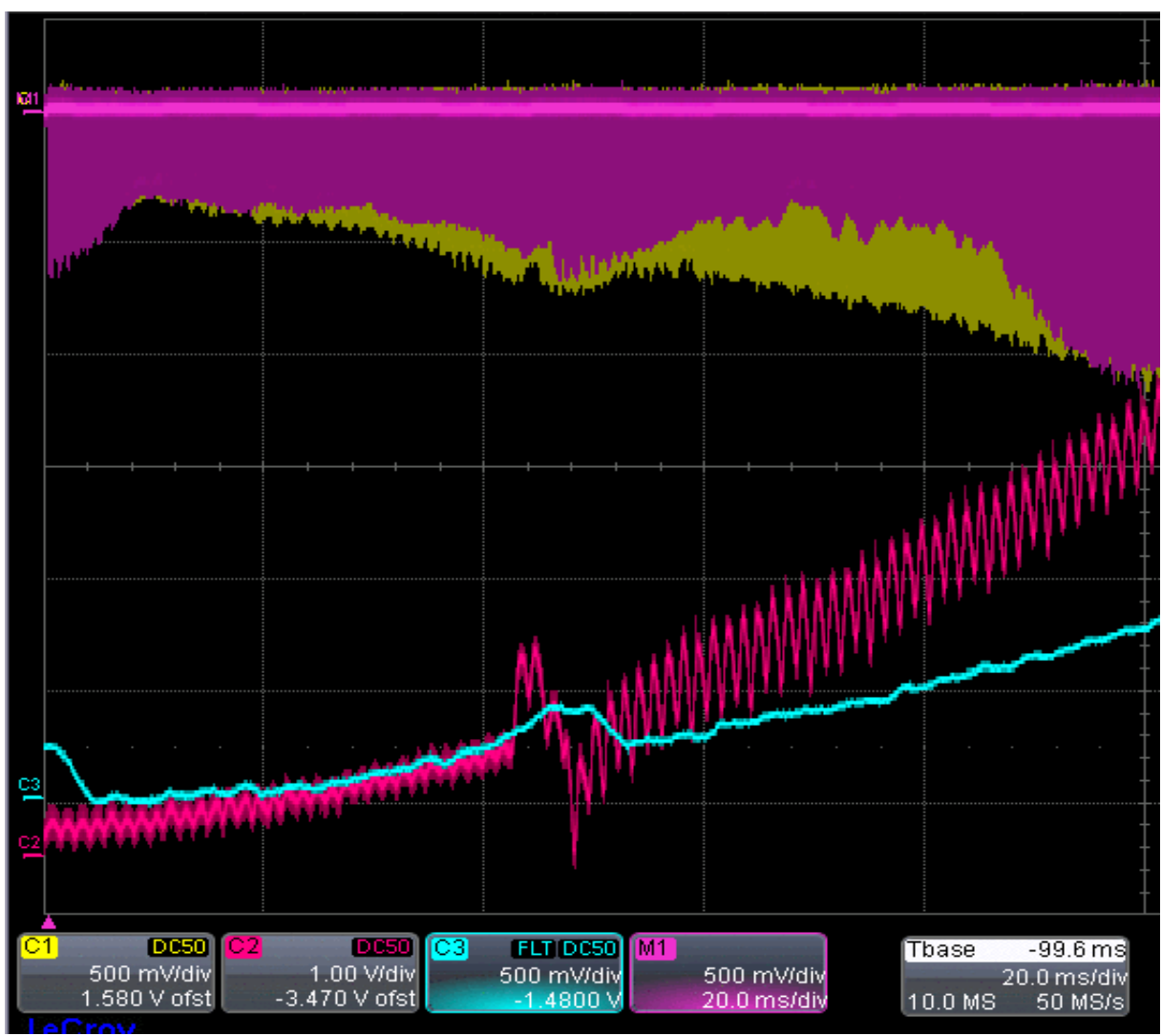

Figure 15: The F to P voltage bank transfer as seen on a magnet voltage signal (red). Also shown are the WCM envelopes with (purple) and without (yellow) the dual harmonic. The blue trace is an $\mathrm{h}=12 \mathrm{Rf}$ voltage signal. This is looking over the first $100 \mathrm{~ms}$ of the ramp.

Note that at near injection the dual harmonic peak current is at least as high as in the case where it is not used. The injected bunch is not matched to the dual harmonic bucket so, at least

\footnotetext{
${ }^{22}$ The voltage applied to the magnets is actually the sum of 2 voltages that are phased to cancel much of this ripple, so the ripple appears to be worse in the figure than it is, but it's still true that there is more voltage ripple after the transfer than before it.
} 
when this picture was taken, the dual harmonic was not active right at injection. In order to use the dual harmonic starting right at injection the bunch coming out of the Booster was subsequently widened using "quad pumping” which induces longitudinal bunch shape oscillations. The bunch is then extracting when it is at its widest. Figure 16 shows the quad pumping in the Booster as viewed on the WCM envelope. Note that extraction occurs when the peak current is at a minimum.

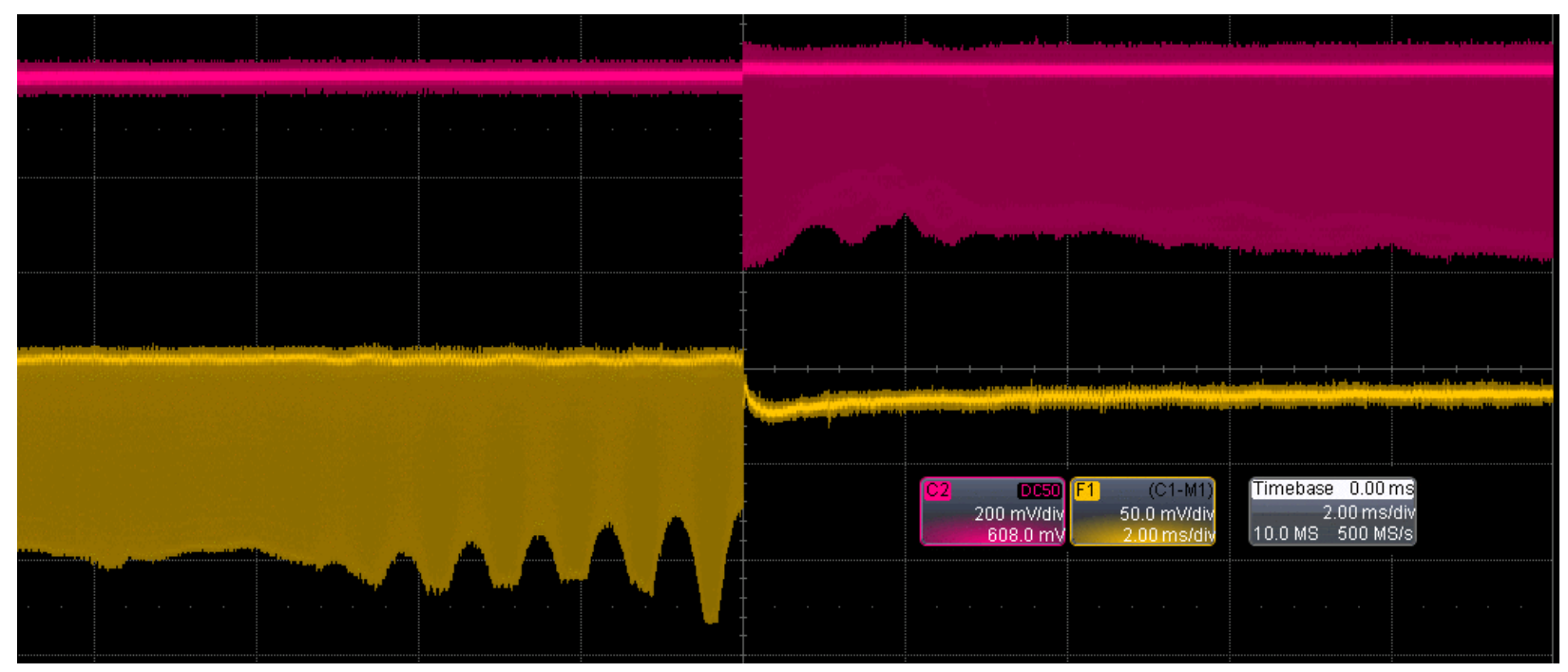

Figure 16: The Booster (orange) and AGS (red) WCMs with quad pumping on near Booster extraction. ${ }^{23}$

Figure 17 shows WCM mountain range displays from injection (At0+144 ms) up until where the $\mathrm{h}=12$ voltage starts to ramp down. ${ }^{24}$ The interval from 144 to $154 \mathrm{~ms}$ shows the injection of the quad pumped bunch into the dual harmonic. In the interval from 184 to $194 \mathrm{~ms}$ the bunch appears to wobble because the synchronous phase is changing a lot due to the $\mathrm{F}$ to $\mathrm{P}$ transfer. The bunch during that period is no longer particularly flat. Afterwards the bunch shape largely recovers though it never becomes as uniform as it was before the transfer. This may not only be due to the abrupt nature of the transfer but also because of the larger magnet voltage ripple after it. Also shown is the WCM envelope from At0+140 to 240 ms. ${ }^{25}$ From that one can also see that the bunch is not as well behaved after the $\mathrm{F}$ to $\mathrm{P}$ transfer, although the peak current more or less returns to a value which is not much greater than what it was before it.

\section{AGS Flattop Emittance with and without the Dual Harmonic}

A scan of transverse emittance vs. the AGS intensity on the flattop was performed on April 7 with and without the dual harmonic. ${ }^{26}$ The intensity was varied by adjusting the Linac pulse width while keeping the scraping in the Booster constant. The Linac current was the typical one, corresponding to 6.9e11 for a $300 \mu$ s pulse. Nominally, the beam extracted from the Booster

\footnotetext{
${ }^{23}$ Booster-AGS-PP 2017 elog Mar 201742 entry

24 Booster-AGS-PP 2017 elog Mar 231847 entry

25 Booster-AGS-PP 2017 elog Mar 201748 entry

26 Booster-AGS-PP 2017 elog Apr 71848 through 2028
} 
will have an emittance independent of injected intensity using this method, so any intensity dependence in the AGS should be attributable solely to processes occurring in the AGS. The 95\% normalized emittance was measured on the AGS flattop using the IPM after the Rf shuts off at At0+1000 ms. Figure 18 shows the results. ${ }^{27}$

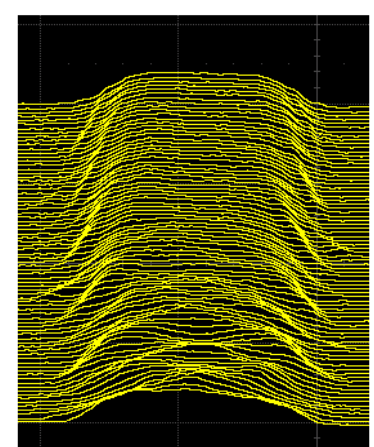

144 to $154 \mathrm{~ms}$

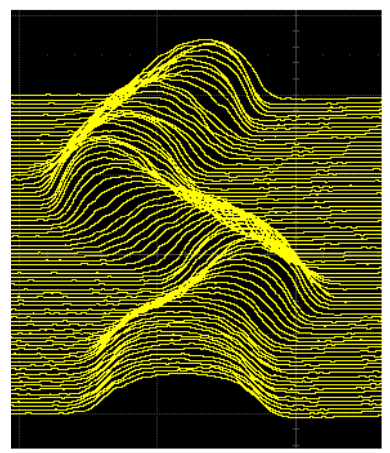

184 to $194 \mathrm{~ms}$

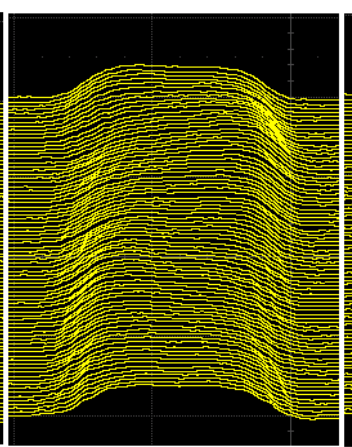

154 to $164 \mathrm{~ms}$

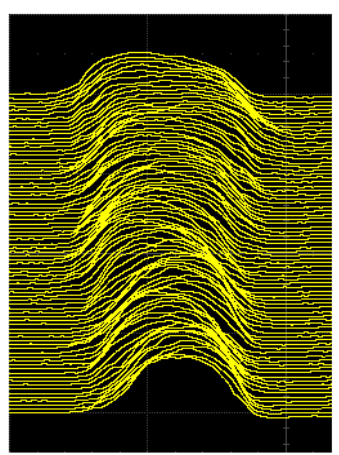

194 to $204 \mathrm{~ms}$

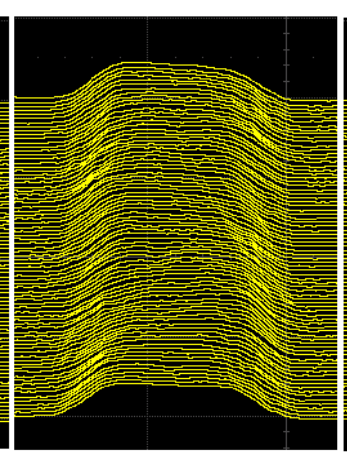

164 to $174 \mathrm{~ms}$

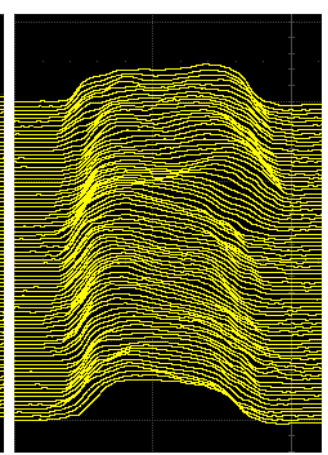

204 to $214 \mathrm{~ms}$

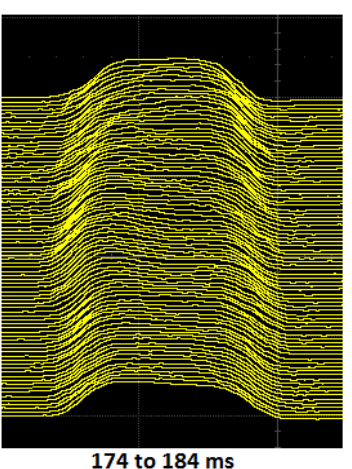

174 to $184 \mathrm{~ms}$

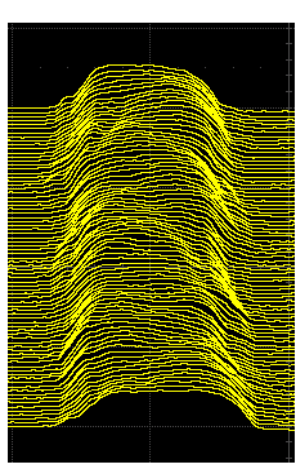

214 to $224 \mathrm{~ms}$
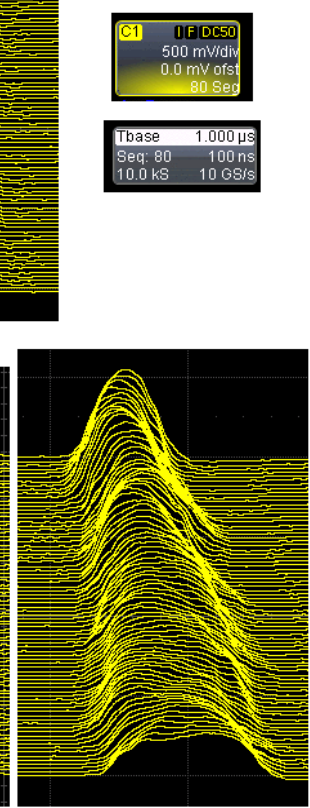

224 to $234 \mathrm{~ms}$

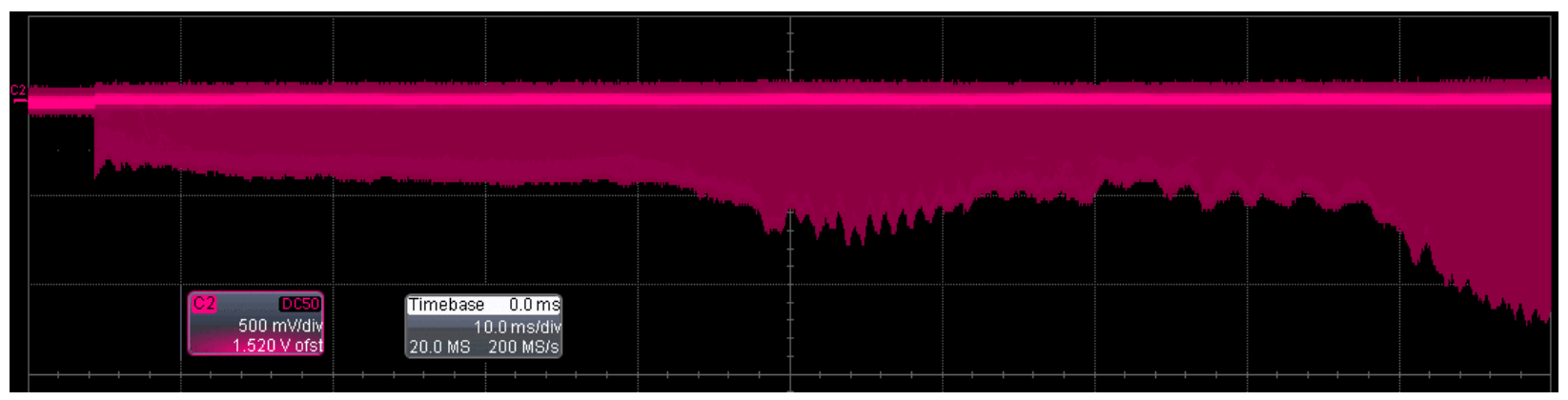

Figure 17: WCM mountain range displays from injection to At0+234 ms (top) and the WCM envelope during roughly the same period (At0+140 to $240 \mathrm{~ms}$ ).

Both the horizontal and vertical $\varepsilon$ show less intensity dependence in the dual harmonic case although the difference is perhaps more pronounced in the vertical. At lower intensities $\varepsilon_{\mathrm{x}}$ is larger for the dual harmonic case and is about the same at a typical filling intensity ( 2.2e11).

\footnotetext{
${ }^{27}$ The values of $\varepsilon_{x}$ and $\varepsilon_{y}$ were found using the logged IPM data during the time of the intensity scan using the method described in footnote 35. For both AU2 and AU4 they were found at 8 different intensities. For the 8 intensities, starting with the lowest, the number of sets of measurements of $\varepsilon_{\mathrm{x}}$ and $\varepsilon_{\mathrm{y}}$ for AU2 were $(19,20,21,17,19,11,16,11)$ and for AU4 they were $(16,16,9,17,17,19,15,17)$.
} 
This may seem odd, but there may be some differences between the 2 setups besides whether the dual harmonic is present or not. For example, the tunes during the early part of the cycle may be slightly different.

For 2.2e11 the plot indicates $\left(\varepsilon_{\mathrm{x}}, \varepsilon_{\mathrm{y}}\right)=(12.5,13.5)$ with the dual harmonic and $(12.5,14.5) \pi \mathrm{mm} \mathrm{mr}$ without it. At 3.0e11 the difference in the vertical is larger than at 2.2e11: $\left(\varepsilon_{\mathrm{x}}, \varepsilon_{\mathrm{y}}\right)$ is $(14.3,15.5)$ with vs. $(15,17.5) \pi \mathrm{mm} \mathrm{mr}$ without the dual harmonic. In both cases the growth seems to start around 1.8e11 and continues at a more or less linear rate, it's just that in the dual harmonic case the rate is lower. ${ }^{28}$

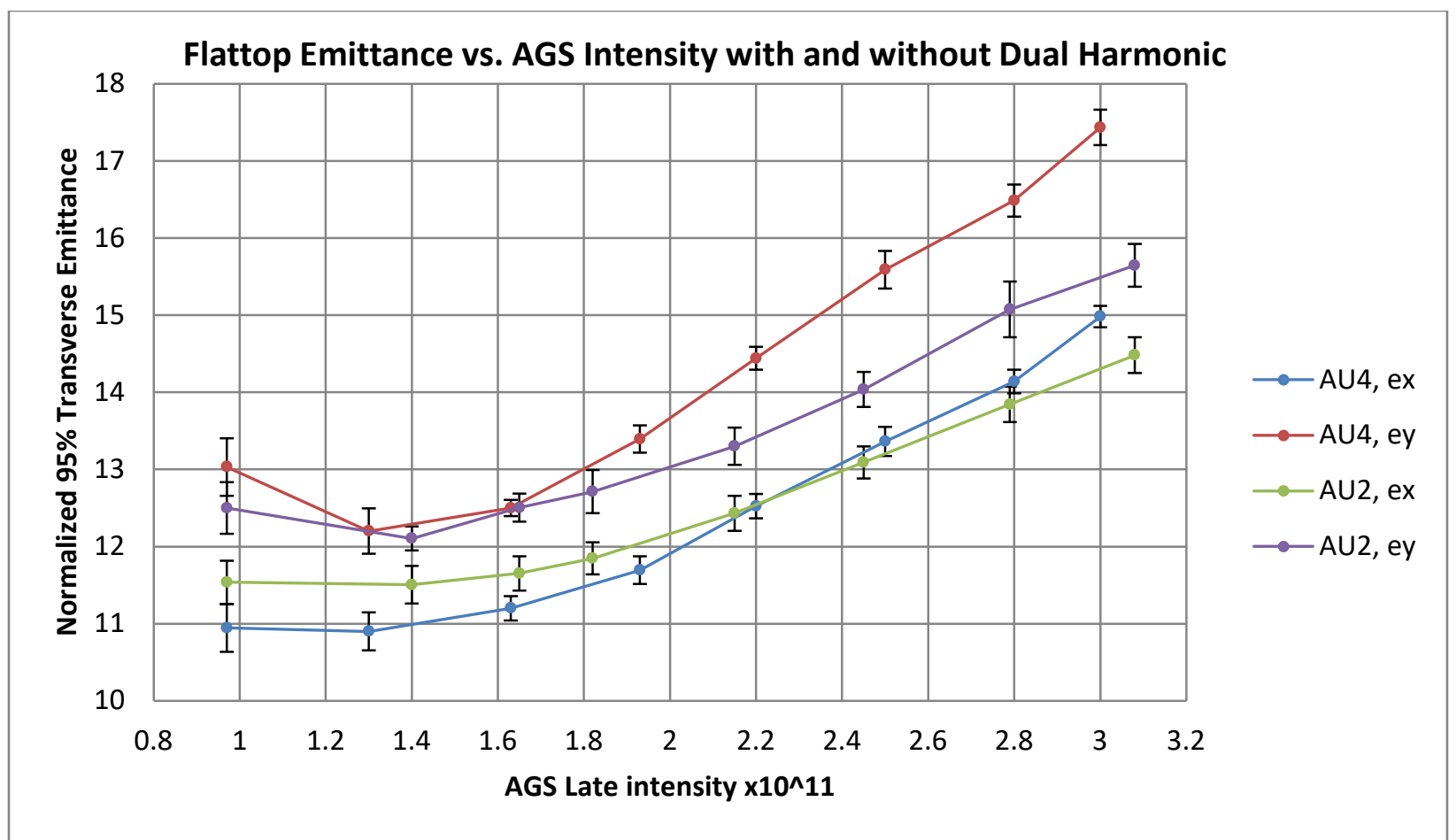

Figure 18: AGS flattop 95\% normalized transverse emittance (in $\pi \mathrm{mm} \mathrm{mr}$ ) vs. AGS intensity at At0+900 ms (AGS Late) using the IPMs with (AGS user 2) and without (AGS user 4) the dual harmonic. $\varepsilon_{\mathrm{x}}$ is horizontal and $\varepsilon_{\mathrm{y}}$ is vertical emittance. The error bars are plus and minus the standard deviation of the measurements.

\section{AGS Flattop Emittance in a Bare Machine Compared to the PP Setup}

In Run 16 an IPM flattop transverse emittance vs. intensity scan was also done but in a bare machine (i.e.- no snakes, $Q_{v}$ far from an integer, no jump quads, etc.). ${ }^{29}$ The intensity was adjusted using the OPPIS Rb temperature and there was no scraping in the Booster. The results are shown in Figure 19 together with the data from the dual harmonic scan shown in Figure 18. Although the range of intensities in the bare machine scan is quite different, there is some

\footnotetext{
${ }^{28}$ Any reduction in the intensity dependence from adjusting the AGS sextupole and octupole correctors has thus far been inconclusive.

${ }^{29}$ Booster-AGS-PP June 272016 elog
} 
overlap. Data from BtA mw006 in the bare machine case are also shown and for this data the entire vertical profile is fit, not just the core.

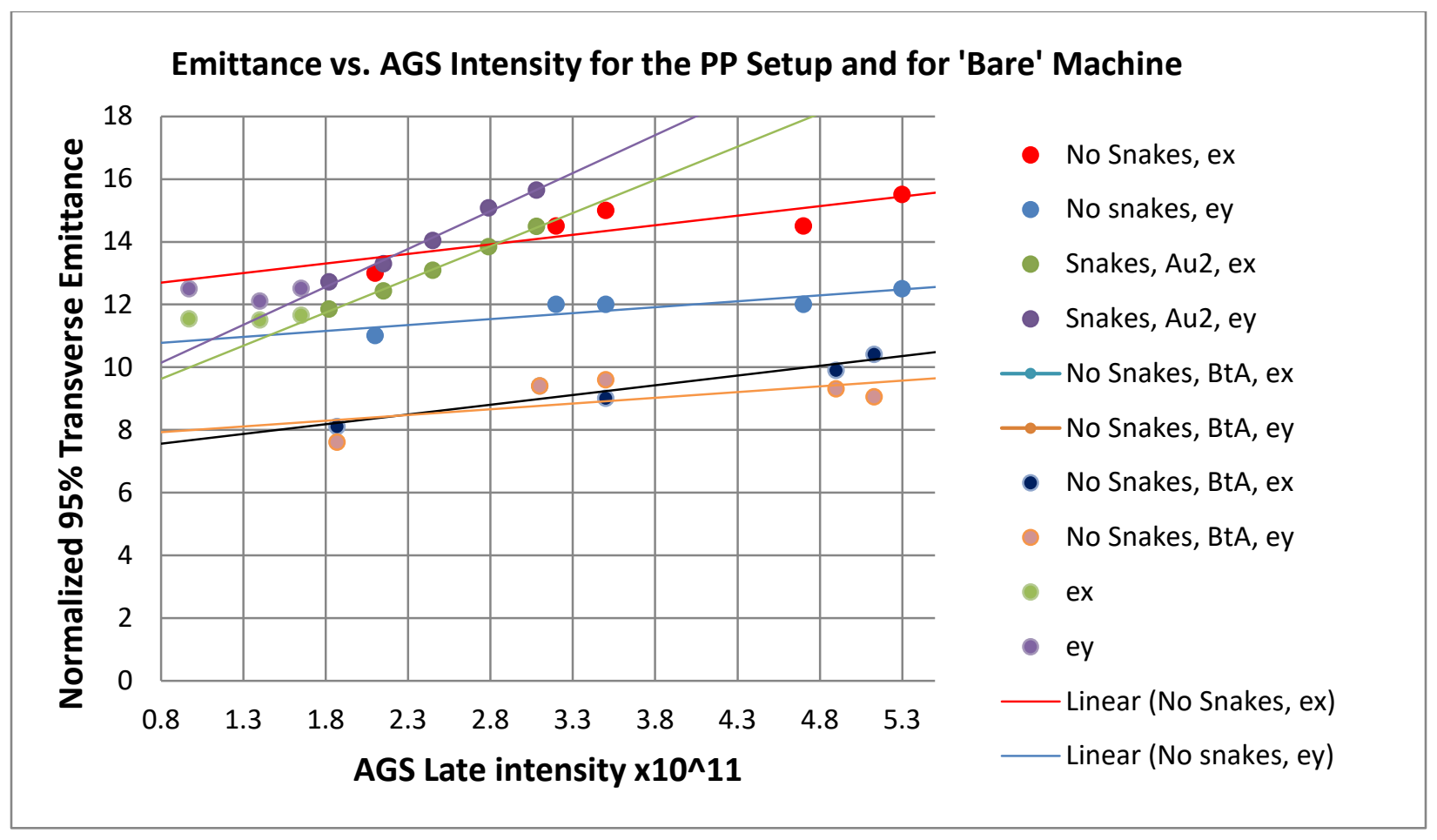

Figure 19: AGS flattop IPM and BtA MW006 95\% transverse normalized emittance (in $\pi \mathrm{mm}$ mr) vs. AGS intensity at At0+900 ms (AGS Late) for bare (No Snakes) machine. Also shown is the dual harmonic data in Figure 18 (labeled Snakes, $\mathrm{Au} 2) . \varepsilon_{\mathrm{x}}$ is horizontal and $\varepsilon_{\mathrm{y}}$ is vertical emittance. For the vertical emittance data in BtA, the full profile is fit to a Gaussian. Linear fits of the data are also shown. In the PP case only the data for AGS late greater than 1.8e11 are fit.

Linear fits to the data are shown, but in the PP case only the data for an AGS late intensity of $1.8 \mathrm{e} 11$ or higher is fit because that's where the intensity dependence seems to begin. The slopes of the linear fits for the bare machine IPM data are much smaller than they are for the PP case and not unlike the slopes for the BtA data. So, it may be that the intensity dependence observed in the AGS for the bare machine case is simply due to growth in the Booster.

In the PP case, even though data for AGS late intensities higher than 3.1e11 don't exist, it seems reasonable to think that the growth would still continue at higher intensities. Recall that for the PP scan the emittances coming out of the Booster are not expected to change with intensity because there is a fixed amount of scraping, so that any intensity dependence should only be due to processes in the AGS.

In the bare machine case, the slopes of the linear fits to the BtA and flattop $\varepsilon_{\text {avg }}$ data are both $0.50 \pi \mathrm{mm}$ mr per 1e11 increase in AGS late. And yet, even though the $\varepsilon$ of the beam coming out of the Booster is likely nearly constant in the PP case, the slope of the linear fit of the PP flattop data (for AGS late $>1.8 \mathrm{e} 11)$ is $(2.27 \pi \mathrm{mm} \mathrm{mr}) / 1 \mathrm{e} 11$, almost 5 times as great. One 
could argue that the bare machine is less intensity dependent because the incoming beam is larger since it's not scraped. Although that possibility can't be completely discounted one might think that there would at least be a sharper trend towards higher $\varepsilon$ at the highest intensities in that case and such a trend isn’t evident.

Still, it is hard to compare the contributions to the final flattop $\varepsilon$ from the intensity dependence of the Booster with that from the AGS in the PP setup because the Booster beam is scraped. But there is Booster late data for the pulse width scans in the previous section. Since the amount of scraping is fixed, if there was any intensity dependence in the $\varepsilon$ of the beam passing through the scrapes the relationship between Booster input and late should start to deviate from linear at the pulse width where that dependence starts, and somewhat surprisingly, it is linear across the range of pulse widths. ${ }^{30}$ So, this data suggests that the Booster's contribution is minimal, although it's likely that for more scraping or higher input nonlinearity would be evident. It is also likely that in this case the Booster was optimized for the full pulse width.

In the bare machine case, $\varepsilon_{\text {avg }}$ on the flattop is about 40 to $50 \%$ larger than it is in BtA. In the PP case, at a low AGS late (where intensity effects are not important), say 1.6e11, the average IPM flattop $\varepsilon$ would be about $10 \pi \mathrm{mm} \mathrm{mr}$ (see Figure 25) for normal running. And in BtA, even for a substantially higher AGS late, $\varepsilon_{\text {avg }}$ is about $5 \pi \mathrm{mm} \mathrm{mr}$ (see Figure 24). So, in the $\mathrm{PP}$ setup $\varepsilon_{\mathrm{avg}}$ is about twice as large on the AGS flattop as it is in BtA. This may in part be due to the fact that an optical injection mismatch with a smaller incoming beam will cause disproportionately more growth than it will with a larger incoming beam. Also, $\varepsilon_{\mathrm{x}}$ is greater than $\varepsilon_{\mathrm{y}}$ in both BtA and on the flattop in the bare machine case, whereas the opposite is typically true on the flattop in the PP running condition.

\section{Booster Input vs. AGS Flattop Polarization}

In Run 16 the nominal Booster input was about 9e11, but it was noticed this run that the AGS flattop and source polarizations may be higher at inputs lower than that. ${ }^{31}$ So on April $4^{\text {th }}$ a rather detailed scan of Booster input vs. AGS flattop polarization at constant AGS intensity was made. ${ }^{32}$ Table VI shows the results, which also include the source polarization and $\varepsilon$ measurements in BtA and on the AGS flattop. The input was varied using the Rb temperature and the amount of scraping in the Booster was adjusted to provide 2.3e11 at AGS late. The AGS polarizations indicated are the average of ten polarization measurements each with $40 \mathrm{e} 6$ events.

For the 2 sets with the lowest input (5.9e11) the AGS polarization is higher than for the other cases, and although the change in the source polarization over this range of inputs is small,

\footnotetext{
${ }^{30}$ Unfortunately, there is no BtA multiwire data for these scans, but as mentioned previously, it seems reasonable to assume there was little or no intensity dependence there because the scraping occurs at a relatively high energy. See here for the AU2 case.

${ }^{31}$ Booster-AGS-PP Apr 12017 elog 1736 and 1758 entries (H. Huang)

32 Booster AGS-PP Apr 42017 elog entries from 1342 to 1810
} 
the higher the input the lower its value. The standard deviations of the source measurements are also smaller than they are for the AGS polarization measurements. On the other hand, the BtA $\varepsilon$ becomes smaller as the input is increased and, to a lesser extent, so does the AGS flattop $\varepsilon$. These measurements would've been taken at even lower Rb temperatures but the input became unstable if it was lowered any further.

Although an input of 5.9e11 had the highest measured polarization, given the significant variation in the measurements and the fact that the measured $\varepsilon$ is somewhat larger at lower inputs, it was decided not to lower the nominal input all the way down to that value, but to lower it from the Run 15 value (9e11) to 7e11. This is about where it was left for the remainder of the run.

\begin{tabular}{|l|l|l|l|l|l|l|l|}
\hline Booster input & BtA $\boldsymbol{\varepsilon}_{\mathbf{x}}$ & BtA $\boldsymbol{\varepsilon}_{\mathbf{y}}$ & $\mathbf{I P M} \boldsymbol{\varepsilon}_{\mathbf{x}}$ & $\mathbf{I P M} \boldsymbol{\varepsilon}_{\mathbf{y}}$ & AGS Pol. & Source Pol. & Rb Temp \\
\hline $5.9 \mathrm{e} 11$ & 9.7 & 3.1 & 12.2 & 13.6 & $69.2 \pm 2.9$ & $85.4 \pm 1.7$ & 75 \\
\hline $5.9 \mathrm{e} 11$ & - & - & - & - & $67.9 \pm 2.1$ & $85.1 \pm 1.6$ & 75 \\
\hline $7.0 \mathrm{e} 11$ & 8.8 & 2.6 & 11.8 & 12.8 & $66.4 \pm 3.6$ & $84.6 \pm 1.0$ & 77.8 \\
\hline $8.8 \mathrm{e} 11$ & 7.6 & 2.3 & 11.8 & 12.8 & $65.9 \pm 1.8$ & $84.1 \pm 1.3$ & 82.1 \\
\hline
\end{tabular}

Table VI: AGS flattop polarization for different Booster inputs. In all cases AGS late was kept at 2.3e11 by varying Booster scraping. The OPPIS source Rb temperature was varied to change the input. Each polarization indicated is the average of ten 40e6 event measurements. Also shown are the source polarization and $\varepsilon$ measurements in BtA (MW006) and on the AGS flattop (IPM). Two sets of polarization measurements were made for the 5.9e11 input case, one at the beginning and one at the end of the study. The 'uncertainty' shown in each of the polarization measurements is the standard deviation of that set of 10 measurements.

\section{The Effect of the Linac Intensity and the H- Stripping Foil on BtA Transverse Emittance}

On June $29^{\text {th }}$ a study was performed to quantify the effect that the stripping foil has on the transverse emittance as measured in BtA. A short Linac pulse ( $70 \mu \mathrm{s})$ was injected at various times with respect to the collapse of the injection bump. The injection bump timing (fast.tm) was adjusted to move where the injected pulse falls on the injection bump.

The timing for the case where the pulse is injected as close to the collapse of the bump as possible was determined by adjusting fast.tm to provide the smallest Gaussian fit profile widths on BtA MW006. The injection bump collapses to about $90 \%$ of its full value in about $50 \mu$ s. With this bump timing the time spent on the foil after the end of the pulse was assumed to be close enough to zero that its effect would be negligible. Therefore, in this case, the average time a particle spent passing through the foil $\left(\mathrm{t}_{\mathrm{avg}}\right)$ would be $35 \mu \mathrm{s}$.

Three sets of Gaussian fits of BtA multiwire profile data were taken for 5 different settings of fast.tm corresponding to $t_{\text {avg }}$ 's of 35, 135, 235, 335, and $445 \mu \mathrm{s}$. Many measurements of the BtA $\varepsilon$ vs. pulse width have been made in the past to find the effect of the foil. One downside to that method is that there may be intensity related effects which contribute to any 
dependence. Using this method, since the pulse width is short and constant it is unlikely there will be any intensity dependent contribution. On the other hand, one potential source of error was already alluded to above, that is, the time it takes for the bump to collapse. Another is that the position and angle of the injection bump may not be exactly constant over the interval studied. Although neither the position nor angle reference for injection bump are exactly flat over the interval, they only change by a percent or 2 .

Table VII shows the data, Figure 20 is a plot of the FWHM ( $\Delta \mathrm{x}$ and $\Delta \mathrm{y})$ data together with linear fits, and Figure 21 is a plot of emittance calculated from the linear fits for $(\Delta x, \Delta y)$ vs. time on the foil. Also shown in the Table is $\Delta \mathrm{x}$ corrected for dispersion and these values for $\Delta \mathrm{x}$ (and $\left.\varepsilon_{\mathrm{x}}\right)$ are the ones shown in the figures. The y-intercepts of the linear fits for $(\Delta \mathrm{x}, \Delta \mathrm{y})$ are $(3.07,6.30) \mathrm{mm}$ which correspond to the widths for no time on the foil (i.e.- $\mathrm{t}_{\mathrm{avg}}=0$ ). Those widths in turn correspond to $\left(\varepsilon_{\mathrm{x}}, \varepsilon_{\mathrm{y}}\right)=(7.70,6.14) \pi \mathrm{mm} \mathrm{mr}$ which is an $\varepsilon_{\mathrm{avg}}$ (i.e.-sqrt $\left.\left[\left(\varepsilon_{\mathrm{x}}{ }^{2}+\varepsilon_{\mathrm{y}}{ }^{2}\right) / 2\right]\right)$ of $6.97 \pi \mathrm{mm}$ mrad.

\begin{tabular}{|c|c|c|c|c|c|c|c|c|}
\hline \multirow{2}{*}{$\begin{array}{l}\text { Time } \\
(\mu \mathrm{s})\end{array}$} & \multicolumn{2}{|c|}{$\Delta x$} & \multirow[t]{2}{*}{$\Delta y$ (total) } & \multirow[t]{2}{*}{$\Delta y$ (core) } & \multicolumn{2}{|c|}{$\varepsilon_{\mathrm{x}}$} & \multirow[t]{2}{*}{$\varepsilon_{\mathrm{y}}$ (total) } & \multirow{2}{*}{$\varepsilon_{y}$ (core) } \\
\hline & with D & no D & & & with D & no D & & \\
\hline 35 & 2.99 & 2.88 & 6.54 & 5.35 & 7.33 & 6.79 & 6.63 & 4.44 \\
\hline 35 & 3.41 & 3.31 & 6.41 & 5.67 & 9.54 & 8.99 & 6.37 & 4.98 \\
\hline 35 & 3.33 & 3.23 & 6.50 & 5.64 & 9.09 & 8.55 & 6.55 & 4.93 \\
\hline 135 & 3.33 & 3.23 & 7.11 & 7.11 & 9.09 & 8.55 & 7.84 & 7.84 \\
\hline 135 & 3.46 & 3.36 & 6.43 & 6.43 & 9.82 & 9.27 & 6.41 & 5.71 \\
\hline 135 & 3.67 & 3.58 & 7.16 & 7.16 & 11.04 & 10.50 & 7.95 & 7.95 \\
\hline 235 & 3.69 & 3.59 & 7.56 & 7.56 & 11.10 & 10.56 & 8.86 & 8.86 \\
\hline 235 & 3.49 & 3.39 & 7.90 & 7.90 & 9.99 & 9.45 & 9.67 & 7.92 \\
\hline 235 & 3.86 & 3.77 & 7.37 & 7.37 & 12.22 & 11.68 & 8.42 & 8.42 \\
\hline 335 & 3.87 & 3.78 & 8.23 & 8.23 & 12.28 & 11.74 & 10.50 & 10.50 \\
\hline 335 & 4.02 & 3.94 & 7.92 & 7.92 & 13.25 & 12.71 & 9.72 & 9.72 \\
\hline 335 & 3.85 & 3.76 & 7.12 & 7.12 & 12.15 & 11.61 & 7.86 & 7.86 \\
\hline 435 & 4.15 & 4.07 & 8.44 & 8.44 & 14.12 & 13.58 & 11.04 & 11.04 \\
\hline 435 & 4.20 & 4.12 & 8.67 & 8.67 & 14.46 & 13.92 & 11.65 & 11.65 \\
\hline 435 & 4.07 & 3.99 & 8.45 & 8.45 & 13.58 & 13.04 & 11.07 & 11.07 \\
\hline
\end{tabular}

Table VII: BtA MW006 Gaussian fit Full Width Half Maximums ( $\Delta \mathrm{x}$ and $\Delta \mathrm{y}$ ) in mm vs. average time on the $\mathrm{H}^{-}$foil. Also shown are 95\% transverse normalized emittance for the horizontal and vertical. In the vertical a Gaussian fit of only the core of the profile as well as the total profile are shown. Two values for the $\Delta \mathrm{x}$ and $\varepsilon_{\mathrm{x}}$ are shown; one of them is uncorrected for dispersion (with $\mathrm{D}$ ) and the other is with the dispersion contribution subtracted from it (no D).

Note also that in Table VII, for the vertical, the fits not only for the entire profile but for just the core as well are shown. For longer times on the foil these values are the same, but for $\mathrm{t}_{\mathrm{avg}}=35 \mu$ s they differ considerably. The fit of the core at $\mathrm{t}_{\mathrm{avg}}=35 \mu$ s corresponds to $4.78 \pi \mathrm{mm}$ $\mathrm{mr}$. Using this value for $\varepsilon_{\mathrm{y}}$ gives an $\varepsilon_{\mathrm{avg}}$ of 6.41 instead of $6.97 \pi \mathrm{mm} \mathrm{mr}$. Perhaps the foil may be spreading out the distribution to something more Gaussian than what it was originally. The 
betatron motion is coupled at Booster injection because both tunes are close to each other, so it's not easy to draw conclusions about the $\varepsilon$ or shape of the injected beam in one plane or the other.

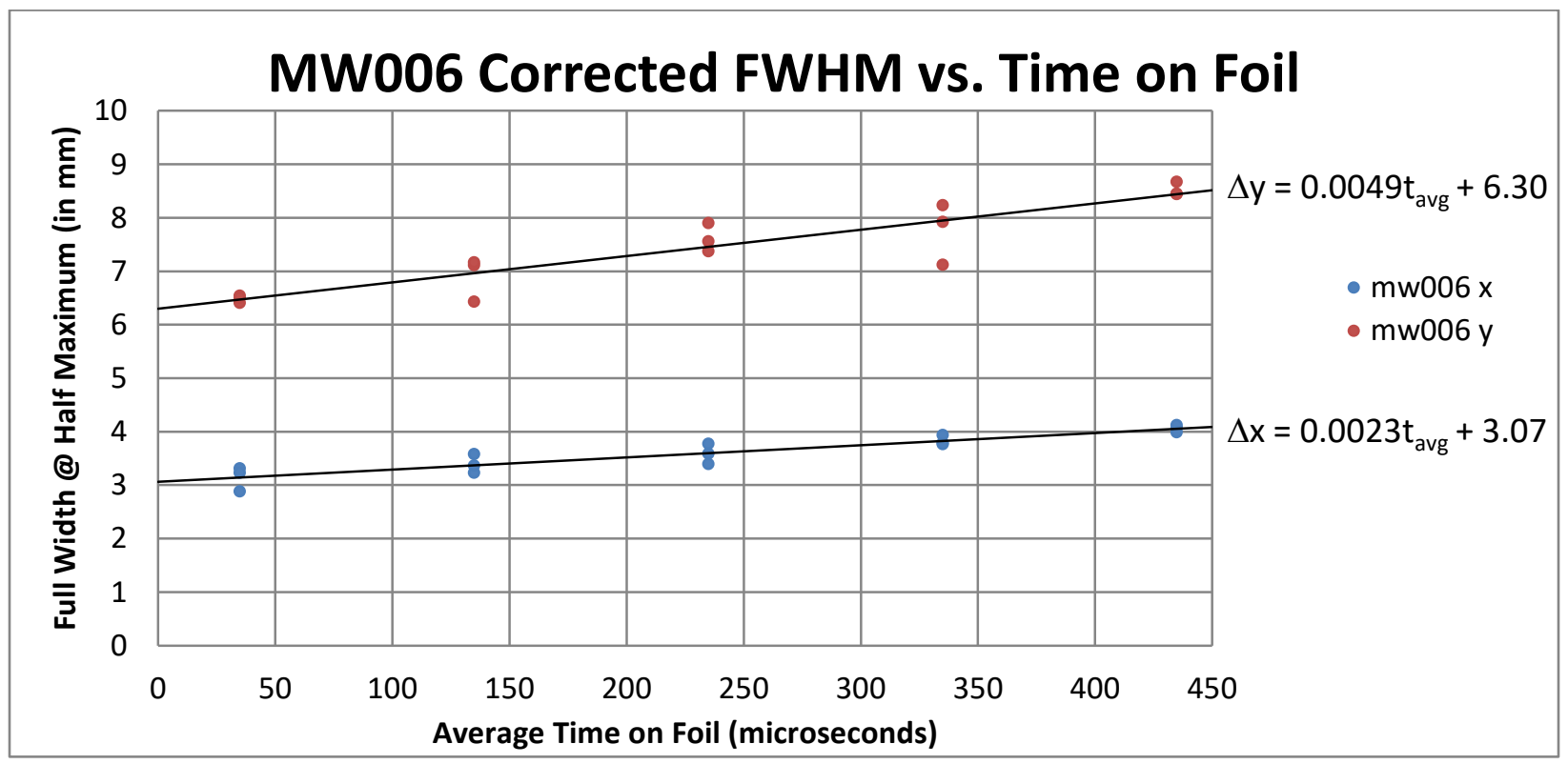

Figure 20: BtA MW006 FWHM Gaussian fit data vs. average time spent of the foil. The horizontal widths $(\Delta \mathrm{x})$ are corrected for dispersion. Also shown are the linear fits to the data.

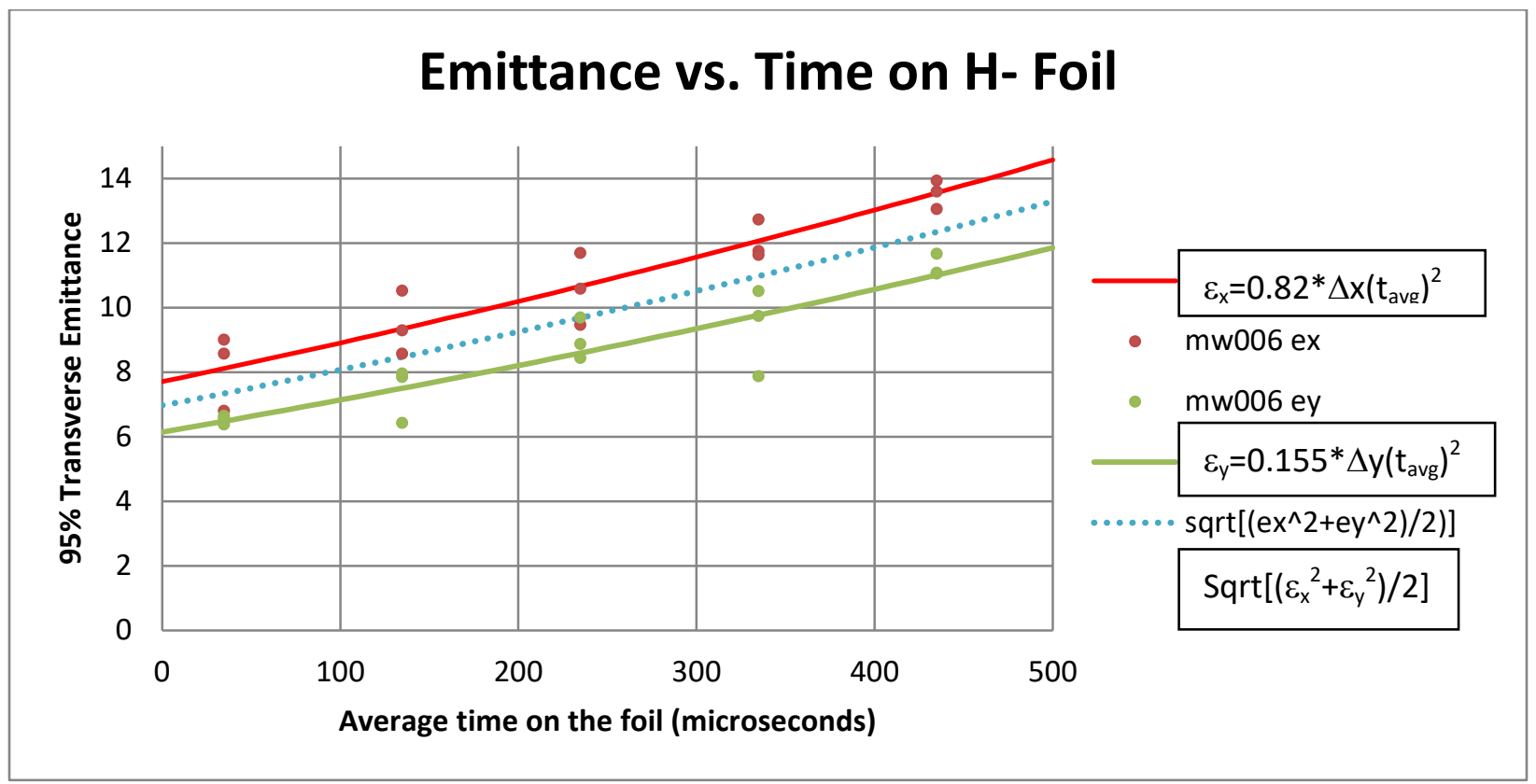

Figure 21: The points shown are $\varepsilon$ values calculated from the corrected $\Delta \mathrm{x}$ and $\Delta \mathrm{y}$ values in Figure 20 . The curves are as described in the legend on the right where $\Delta \mathrm{x}\left(\mathrm{t}_{\text {avg }}\right)$ and $\Delta \mathrm{y}\left(\mathrm{t}_{\text {avg }}\right)$ are the FWHMs for $\mathrm{t}_{\text {avg }}$ calculated from the linear fits shown in figure 20.

Regardless, when Gaussian fits of the vertical profiles in LtB are performed, a fit of the core is also typically narrower than a fit of the entire profile and the horizontal profiles more 
closely resemble a Gaussian as in BtA. Figure 22 shows the same vertical profile with the core fit (top left) and with the entire profile fit (top right) for the $t_{\mathrm{avg}}=35 \mu$ s case on BtA mw006. ${ }^{33}$ The bottom 2 traces show the same vertical profile from LtB mw107 with the core fit (bottom left) and entire profile fit (bottom right). ${ }^{34}$
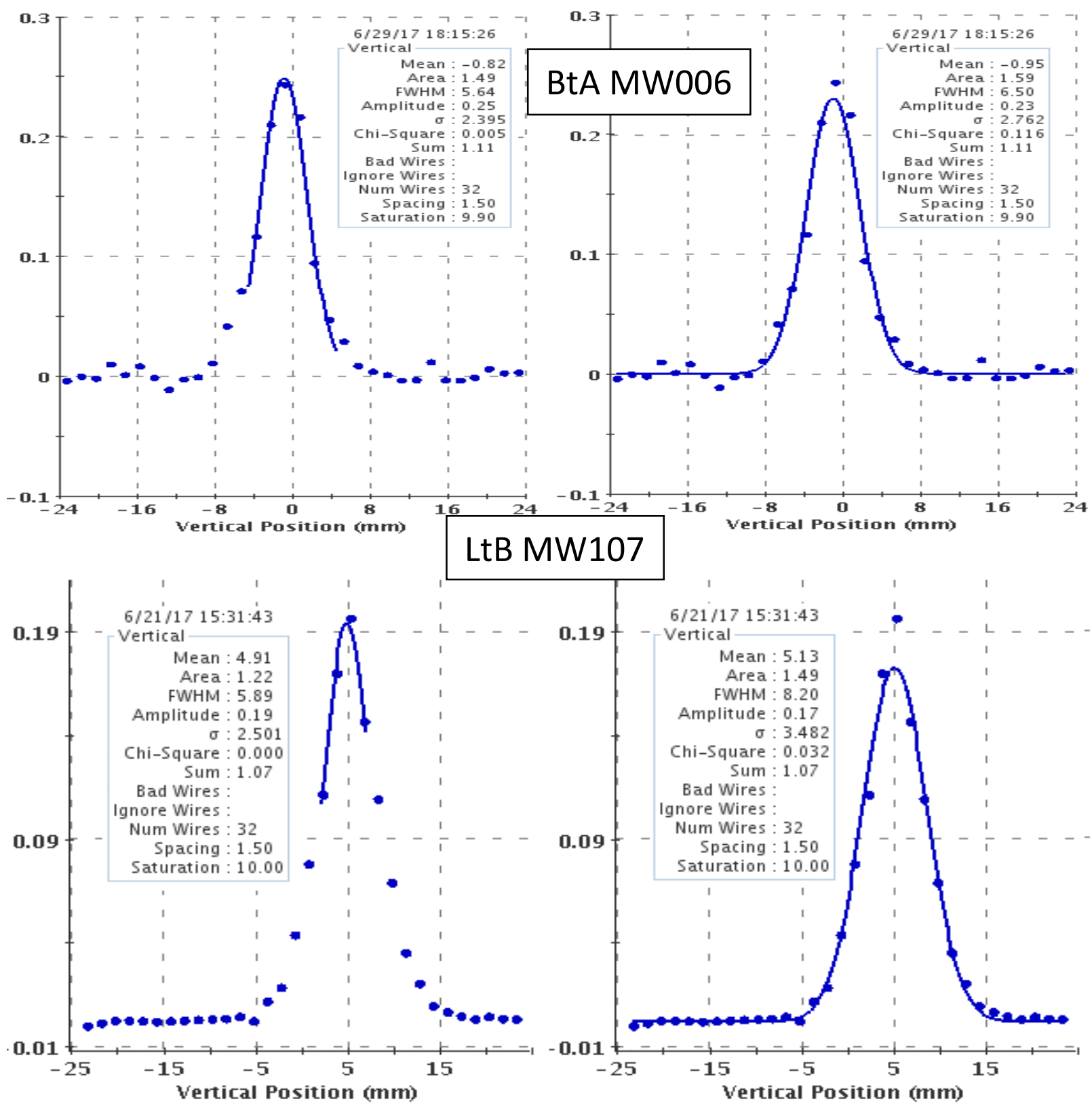

Figure 22: The same vertical profile on BtA MW006 (top) for the $t_{a v g}=35 \mu$ s case with only the core fit (left) and the entire profile fit (right). Below is a vertical profile from LtB MW107 with a fit of the core (left) and a fit of the entire profile (right).

\footnotetext{
${ }^{33}$ Booster-AGS-PP Jun 292017 elog 1818 entry

34 Booster-AGS-PP Jun 212017 elog 1531 entry
} 
Figure 23 shows plots of two pulse width scans, one from May $11^{\text {th }}$ at the nominal Linac current ( $7 \mathrm{e} 11$ for $300 \mu$ s pulse) and the other from May $25^{\text {th }}$ at higher current $(\sim 11.0 \mathrm{e} 11$ for a $300 \mu$ pulse). Superimposed on these plots are the fits of the data and $\varepsilon$ curves from Figure 21 (which are only intended as references). The horizontal widths were corrected for dispersion in the same way they were for the constant pulse width scan. The fits for the May $11^{\text {th }}$ data are linear and those for the May $25^{\text {th }}$ are parabolic because they give the most sensible fits to the data.

Note that in both cases the $\varepsilon$ for the vertical core fit remains different from the fit for the entire profile. Recall that in the case where the pulse width is constant and the injection bump timing is varied the $\varepsilon$ of the core becomes the same as the full $\varepsilon$ when $t_{\text {avg }}$ is $135 \mu$ s or more. Presumably with enough time on the foil the profile loses this feature. But in measurements where the pulse width is varied there is always part of the beam injected just before the bump collapses, so perhaps this feature remains visible.

In the lower current case $\varepsilon_{\mathrm{x}}$ does not show much pulse width dependence, and $\varepsilon_{\mathrm{y}}$ for the entire profile does show some (at least if the first point at $50 \mu$ s is ignored) and the core $\varepsilon_{\mathrm{y}}$ shows even less. ${ }^{35}$ In the higher current case all three sets of profiles show pulse width dependence. ${ }^{36}$ In the lower intensity case the $\varepsilon$ in both planes seems to increase more slowly than the references from Figure 21, but in the higher intensity case they increase at least as quickly. In the case where the only factor is time on the foil it's not obvious that the $\varepsilon$ growth wouldn't be slower than in the references since $t_{\text {avg }}$ for a $300 \mu$ s long pulse is only $150 \mu$ s. But the fact that it is different in the two cases suggests something more is going on.

Space charge effects in the Booster are generally greater in the vertical plane and the core has the highest charge density. If it's true that the narrower vertical core results from the last part of the beam injected, the fact that it does not seem to get much wider in the lower intensity case suggests that, at least for this scan, which uses a $300 \mu$ s 7e11 pulse there is not much of a space charge effect. ${ }^{37}$ Alternately, the fact that it does get significantly wider in the higher intensity case suggests that for a $300 \mu \mathrm{s} 11.0 \mathrm{e} 11$ pulse there is a noticeable space charge effect.

Since the pulse width in the first study was short $(70 \mu \mathrm{s})$ one might expect the foil's effect on the vertical profile to be minimal. Assuming this and that the vertical profile of the incoming beam is Gaussian and matched to the Booster, then in the case where the injected beam is placed right before the collapse of the bump the profile in BtA should also be Gaussian. So the

\footnotetext{
${ }^{35}$ See Booster-AGS-PP May 1120171740 entry for the May 11 scan. The data for the 50 us case look quite clean, so ignoring it can't be justified because the data is too noisy.

${ }^{36}$ See Booster-AGS-PP May 252017 for the May 25 scan. Note that before the measurements were taken the Booster was optimized for higher than normal intensity.

${ }^{37}$ A less detailed scan at 7e11 was taken on Jun 22 . The results were fairly similar except that the vertical core $\varepsilon$ did noticeably increase with longer pulse width (see Booster-AGS-PP Jun 2220171901 entry ).
} 
fact that it still has a narrower core suggests that the incoming beam may not be Gaussian, just as the LtB multiwires show.
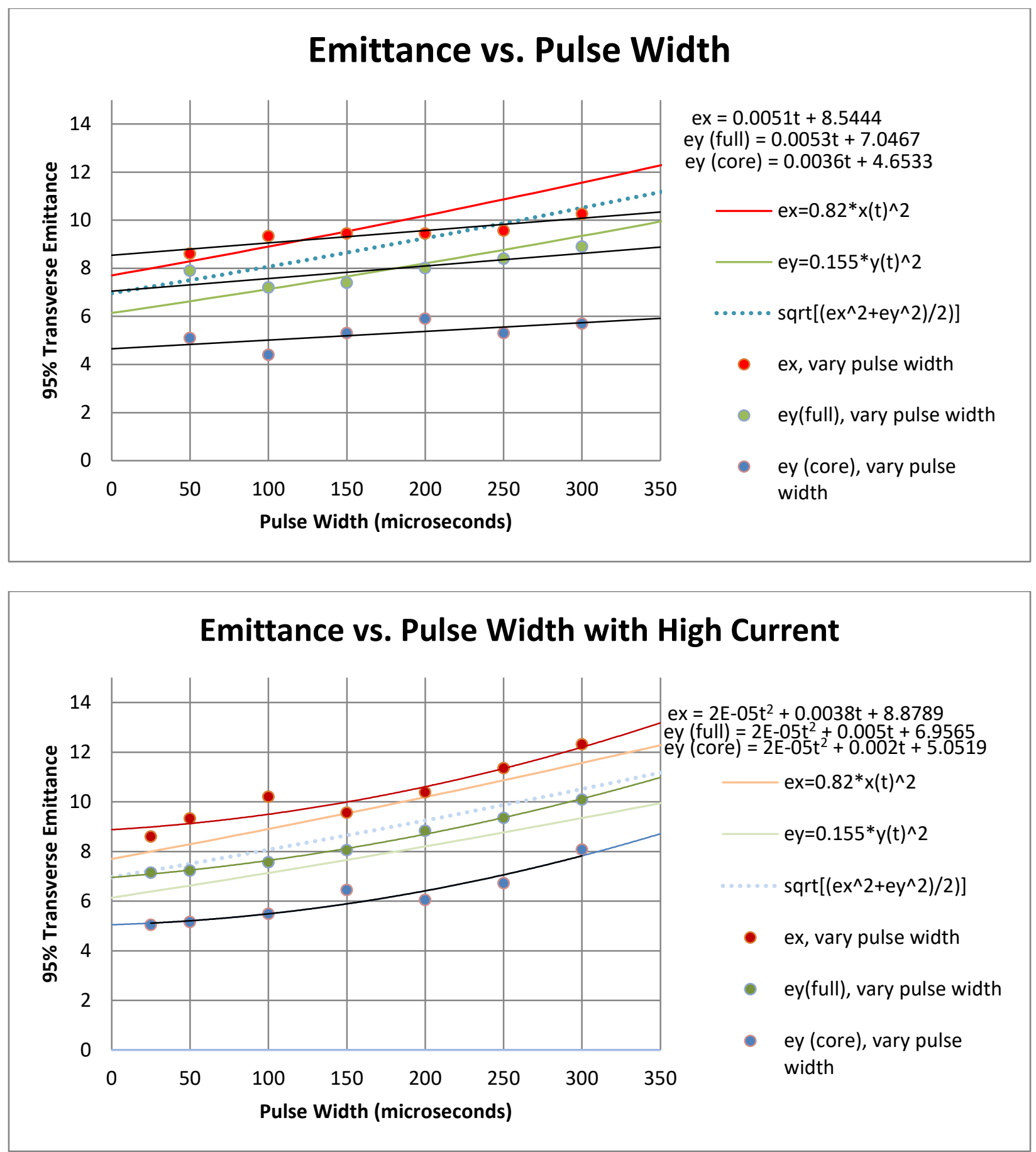

Figure 23: Pulse width scans vs. $\varepsilon$ on BtA MW006. The $\varepsilon$ curves from Figure 21 are also shown. In the top plot (from May 11) the Booster input for the nominal $300 \mu$ s pulse width was 7.1e11 (6.0e11 Booster late) and in the bottom (from May 25) it was 11.0e11 (9.0 Booster late). Also shown are fits to the data which are linear in the top plot and parabolic in the lower one. 
Also, the observation that the narrow core disappears after the beam has been on the foil for $\sim 135 \mu$ s is consistent with this idea since one would expect the narrow core to be lost due to scattering in the foil at some point. It is particularly interesting that the data for the cases where the pulse width is varied are consistent with this in that the core remains regardless of pulse width.

For the May $11^{\text {th }}$ data the fits are shown in Figure 23. The y-intercept for $\varepsilon_{\mathrm{x}}$ is 8.5 , for $\varepsilon_{\mathrm{y}}$ (full) it's 8.18, and for $\varepsilon_{\mathrm{y}}$ (core) it's $4.7 \pi \mathrm{mm}$ mrad. Using $\varepsilon_{\mathrm{x}}$ and $\varepsilon_{\mathrm{y}}$ (full) gives an average $\varepsilon$ of $7.8 \pi \mathrm{mm} \mathrm{mr}$ and using $\varepsilon_{\mathrm{y}}$ (core) gives an average $\varepsilon$ of $6.9 \pi \mathrm{mm} \mathrm{mr}$.

For the May $25^{\text {th }}$ data the y-intercepts are $\varepsilon_{\mathrm{x}}=8.9, \varepsilon_{\mathrm{y}}($ full $)=7.0$, and $\varepsilon_{\mathrm{y}}$ (core) $=5.1 \pi \mathrm{mm}$ mrad. The average $\varepsilon$ using $\varepsilon_{\mathrm{y}}$ (full) is 8.0 and using $\varepsilon_{\mathrm{y}}$ (core) it is $7.2 \pi \mathrm{mm} \mathrm{mr}$. The average $\varepsilon$ values for these 2 sets of data are somewhat larger than the values obtained using the constant pulse width method (6.97 and $6.41 \pi \mathrm{mm} \mathrm{mr}$ for the full and core $\varepsilon_{\mathrm{y}}$ values respectively). Table VIII summarizes these results. In all these cases the strip foil (\#6), which is the one normally inserted, was used.

\begin{tabular}{|c|c|c|c|c|c|}
\hline Date & $\varepsilon_{\mathrm{x}}$ & $\varepsilon_{\mathrm{y}}($ full) & $\varepsilon_{\mathrm{y}}$ (core) & $\varepsilon_{\text {avg }}$ with $\varepsilon_{\mathrm{y}}$ (full) & $\varepsilon_{\text {avg }}$ with $\varepsilon_{\mathrm{y}}$ (core) \\
\hline Apr 4, constant width & 7.7 & 6.1 & 4.8 & 7.0 & 6.4 \\
\hline May 11, input 7e11 & 8.5 & 7.1 & 4.7 & 7.8 & 6.9 \\
\hline May 25, input 11e11 & 8.9 & 7.0 & 5.1 & 8.0 & 7.2 \\
\hline
\end{tabular}

Table VIII: Summary of projected emittances without the effect of the foil for the three scans considered (except for the Apr 4 case where the $\varepsilon_{y}$ (core) is from the core fit width at $35 \mu \mathrm{s}$ ).

\section{Transverse Emittances during Run 17}

Figure 24 shows the transverse emittances as measured on BtA mw006 during more or less nominal running conditions for the part of the run where the dual harmonic was active in the AGS (from March $10^{\text {th }}$ onward). ${ }^{38}$ Note that the nominal Booster input changes from 8e11 to $7 \mathrm{e} 11$ on March $31^{\text {st }}$. The horizontal width seems to decrease shortly after that change (around April 7). This may have to do with a couple of changes made at Linac that day. First, an Rf

\footnotetext{
${ }^{38}$ This data was compiled from BtA mw006 profiles and AGS IPM flattop data in the Booster-AGS-PP run 17 elog. It should be noted that the IPM measurement is not straightforward. The RF is shut off at $1000 \mathrm{~ms}$, and the beam debunches, which reduces the space charge effect allowing for a more accurate measurement. In the horizontal, the reported $\varepsilon_{\mathrm{x}}$ drops reaching a minimum around $1050 \mathrm{~ms}$, then the beam starts to rebunch and the reported $\varepsilon_{\mathrm{x}}$ begins to increase. The minimum value is what's used for $\varepsilon_{x}$. In the vertical, the minimum is reached around 1100 ms and the reported $\varepsilon_{y}$ does not generally increase significantly after that. The average value of $\varepsilon_{y}$ after it reaches its minimum is what's generally reported here. This is nothing particularly new but the rebunching effect does seem to be somewhat more of an issue than it was in Run 15. The blowup in the horizontal doesn't occur with low intensity (for example, March 102149 entry). In that case $\varepsilon_{x}$ remains constant once the Rf is off and it still takes $\varepsilon_{y}$ until $1100 \mathrm{~ms}$ to get to its minimum (this may have to do with the collapse of the extraction bump). In this case after $1100 \mathrm{~ms} \varepsilon_{\mathrm{x}}$ is still near its minimum and so is $\varepsilon_{\mathrm{y}}$, which supports the idea that this method for measuring both is valid.
} 
adjustment was made which made the pulse slightly wider and brought the input up to about $7.4 \mathrm{e} 11$, then the beam was collimated near the source to lower it back to $7 \mathrm{e} 11 .^{39}$

The data in June were generally with a lower intensity because the RHIC filling intensity was lower, and the lack of data in late May and early June was because RHIC was using Gold. In Run $15, \varepsilon_{\mathrm{x}}$ was about 7 and $\varepsilon_{\mathrm{y}}$ was about 2 to $3 \pi \mathrm{mm} \mathrm{mr}$, so not too different. ${ }^{40}$ In Run 15 the input was typically 9e11 and the nominal AGS late intensity was somewhat higher.

Figure 25 shows the AGS IPM emittances on the flattop with the Rf off for the same period. A typical filling intensity was about 2.2-2.3e11, typical transverse emittances were about $\left(\varepsilon_{\mathrm{x}}, \varepsilon_{\mathrm{y}}\right)=(12,13.5)$, and a typical average $\varepsilon$, sqrt $\left[\left(\mathrm{e}_{\mathrm{x}}{ }^{2}+\mathrm{e}_{\mathrm{y}}{ }^{2}\right) / 2\right]$, was about 12.5 to $13 \pi \mathrm{mm} \mathrm{mr}$. In Run 15, the average $\varepsilon$ was about $14.5 \pi \mathrm{mm} \mathrm{mr}$ but the typical AGS late intensity was a little higher, maybe 2.3 to $2.5 \mathrm{e} 11$. $^{41}$

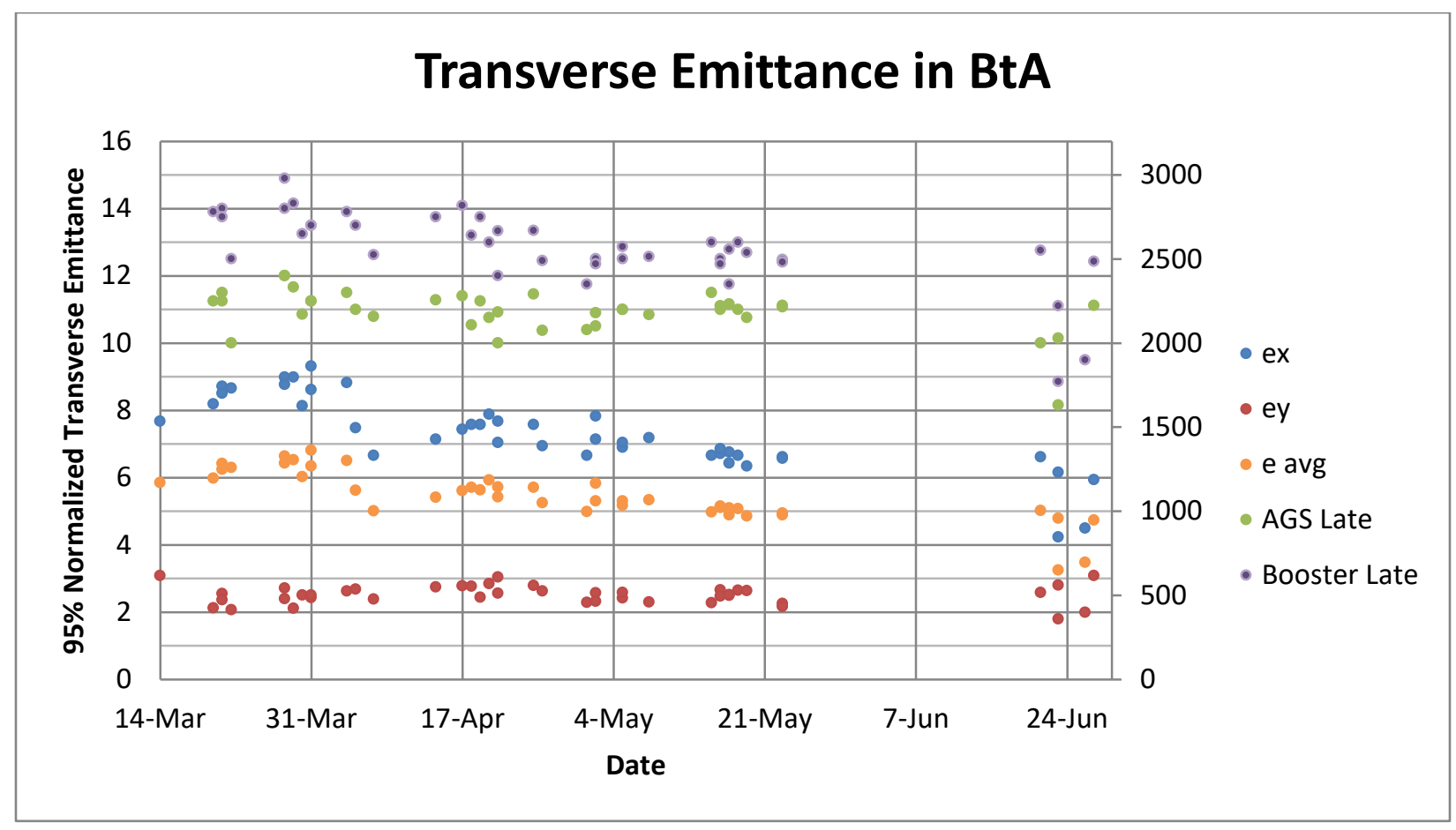

Figure 24: Transverse Emittances as measured from Gaussian fits of BtA mw006 profiles during the run. $\varepsilon_{\mathrm{x}}$ (blue), $\varepsilon_{\mathrm{y}}$ (red), and the average $\varepsilon=\operatorname{sqr}\left[\left(\mathrm{e}_{\mathrm{x}}{ }^{2}+\mathrm{e}_{\mathrm{y}}{ }^{2}\right) / 2\right]$, in orange, are shown. Also shown are Booster (purple) and AGS (green) late intensities, which use the right hand side y-axis where 1000 is $1 \mathrm{e} 11$. The $\varepsilon_{\mathrm{x}}$ values shown here are not corrected for dispersion.

Figure 26 shows the average BtA and AGS IPM flattop emittances (i.e.- $\operatorname{sqrt}\left[\left(\mathrm{e}_{\mathrm{x}}{ }^{2}+\mathrm{e}_{\mathrm{y}}{ }^{2}\right) / 2\right]$ ) compiled from the elogs in Runs 13, 15, and 17 vs. the AGS late intensity. All the IPM data from Run 17 are with the AGS dual harmonic on. For Run 17, the IPM data represents normal running

\footnotetext{
${ }^{39}$ See Booster-AGS-PP elog 2049 entry.

40 See "An Overview if Booster and AGS Polarized Proton Operation During Run 15", K. Zeno, page 16.

${ }^{41}$ See "An Overview if Booster and AGS Polarized Proton Operation During Run 15", K. Zeno, page 28.
} 

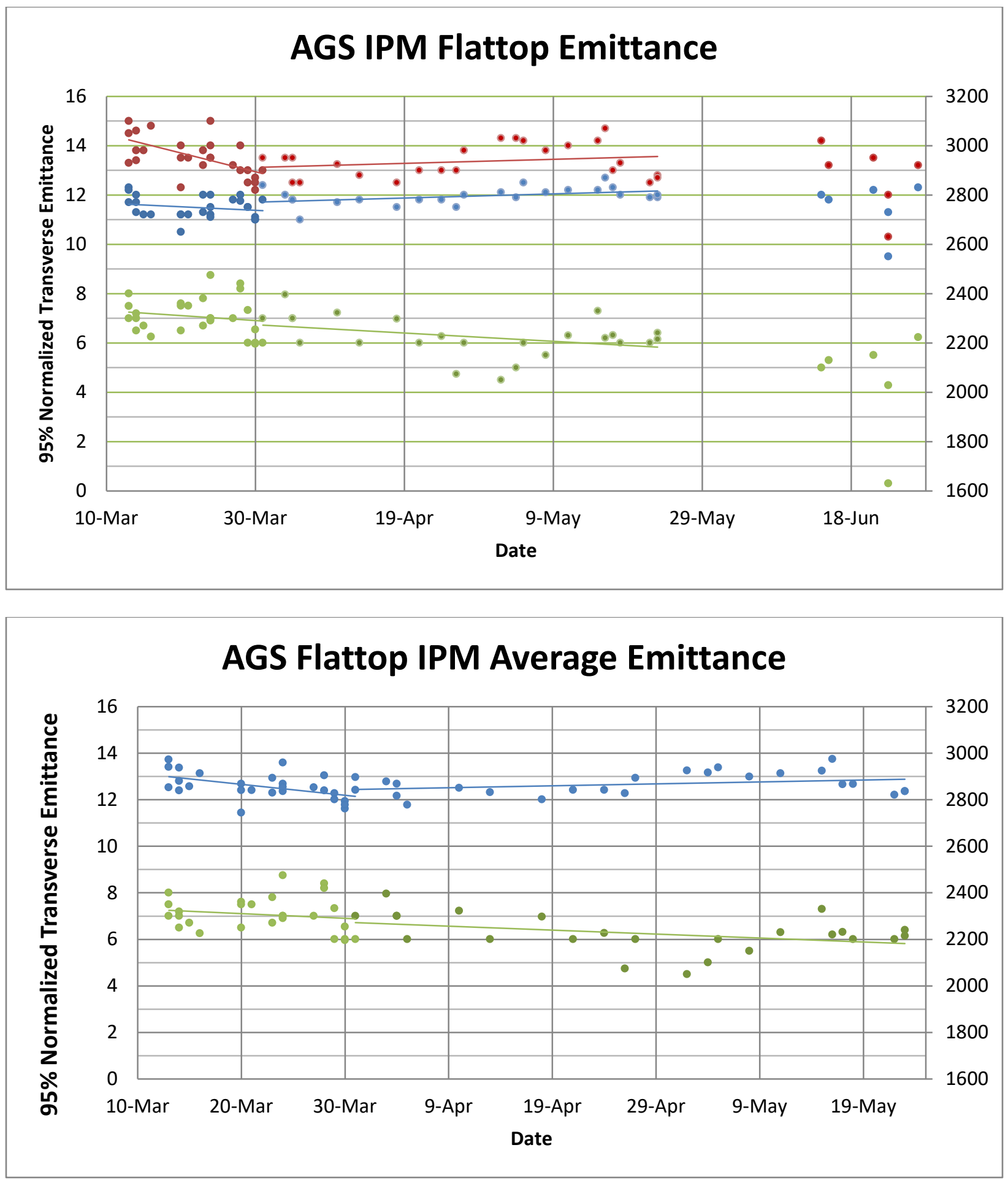

Figure 25: Nominal AGS flattop transverse emittances (in $\pi \mathrm{mm} \mathrm{mr}$ ) as measured from the IPM (with Rf off) during the run. In the top plot, $\varepsilon_{\mathrm{x}}$ (blue), $\varepsilon_{\mathrm{y}}$ (red), and the AGS late intensity (green, using right hand side y-axis) are shown. The bottom plot shows the average $\varepsilon$ (blue) $=\operatorname{sqrt}\left[\left(\varepsilon_{\mathrm{x}}{ }^{2}+\varepsilon_{\mathrm{y}}{ }^{2}\right) / 2\right]$ and the AGS late intensity (green, using right hand side y-axis). The bottom plot does not show data for June. Linear fits of the data are also shown, one for the period before the input change from 8 to $7 \mathrm{e} 11$ and one for after it. For the intensity data 1000 equals $1 \mathrm{e} 11$ protons. 
conditions, except for intensities that are much lower or higher than normal. For example, data from the April $7^{\text {th }}$ intensity scan discussed earlier is included in it.

In Run 13 the Booster input intensity was much lower than in the latter runs ( 4-5e11) so it is not surprising the BtA average $\varepsilon$ is larger. The Run 15 and 17 BtA average $\varepsilon$ is about the same, but the linear fit suggests there is more intensity dependence this year. This may just be due to the lack of multiwire data outside the range of normal running intensities. The AGS flattop average $\varepsilon$ seems to be a couple $\pi \mathrm{mm}$ mr smaller than in Run 15, but if the intensity dependence is different, as one might expect it to be given the results of the April $7^{\text {th }}$ study which showed less dependence with the dual harmonic on, it's not obvious from this plot.

Figure 27 shows transverse emittance data on the Flattop for the ion IPM (the one discussed thus far) and the new eIPM. The eIPM measurements were obtained from the FDAView application and are for blue fills during Run 17 (this includes data prior to March 10). ${ }^{42}$ Some of the data for lower intensities has been removed because it was clearly bad. The ion IPM data is the data used to calculate the average $\varepsilon$ in Figure 26 for Run 17.

The green and orange data are $\varepsilon_{\mathrm{x}}$ and $\varepsilon_{\mathrm{y}}$ for the ion IPM, respectively. And the blue and red data are $\varepsilon_{\mathrm{x}}$ and $\varepsilon_{\mathrm{y}}$ for the eIPM, measured near extraction time. For the eIPM $\varepsilon_{\mathrm{x}}$ is generally larger than $\varepsilon_{\mathrm{y}}$, the opposite of what the ion IPM indicates. Curiously, for the nominal intensity of about 2.2-2.3e11, $\varepsilon_{\mathrm{x}}$ using the ion IPM is about the same as $\varepsilon_{\mathrm{y}}$ using the eIPM and $\varepsilon_{\mathrm{x}}$ for the eIPM is about the same as $\varepsilon_{y}$ for the ion IPM. The names in FDAView indicate that the data is taken near extraction time, and the IPM data is taken after 1000 ms. Perhaps that difference could help explain the discrepancy. The slopes of the linear fits suggest that there is more intensity dependence indicated with the eIPM.

\section{Longitudinal Emittance during Run 17}

A couple longitudinal $\varepsilon$ measurements were made at AGS injection during the run. The first measurement, performed early in the run, was 0.80 ev-sec and it was obtained from the measured first turn width and the synchrotron frequency when the Rf voltage was matched to the beam. This was before the dual harmonic and quad pumping were implemented. ${ }^{43}$ The second, made in May, used the first turn width and the Booster Rf voltage at extraction found from the quad pumping frequency at Booster extraction. ${ }^{44} \mathrm{~A}$ value of 0.71 ev-sec was obtained.

Quite a few measurements of the bunch length on the flattop were made during the run. From these measurements and the synchrotron frequency $\varepsilon_{\text {long }}$ is calculated. The synchrotron frequency was measured for about half of these measurements. Given that that the flattop voltage

\footnotetext{
${ }^{42}$ Specifically the elPM data shown are called agsHEmitAtExtract_elpm and agsVEmitAtExtract_elPM in FDAView and they are plotted against blueAgsXcbmAvg.

${ }^{43}$ See Booster-AGS-PP Jan 92017 elog 1922 entry

${ }^{44}$ See Booster-AGS-PP May 520171346 to 1359 entries
} 
was nearly constant over the run it is not expected to vary much so it was assumed to be a typical value $(120.5 \mathrm{~Hz})$ for the length measurements where it wasn't measured. Table IX shows the data and it is plotted in Figure 28.

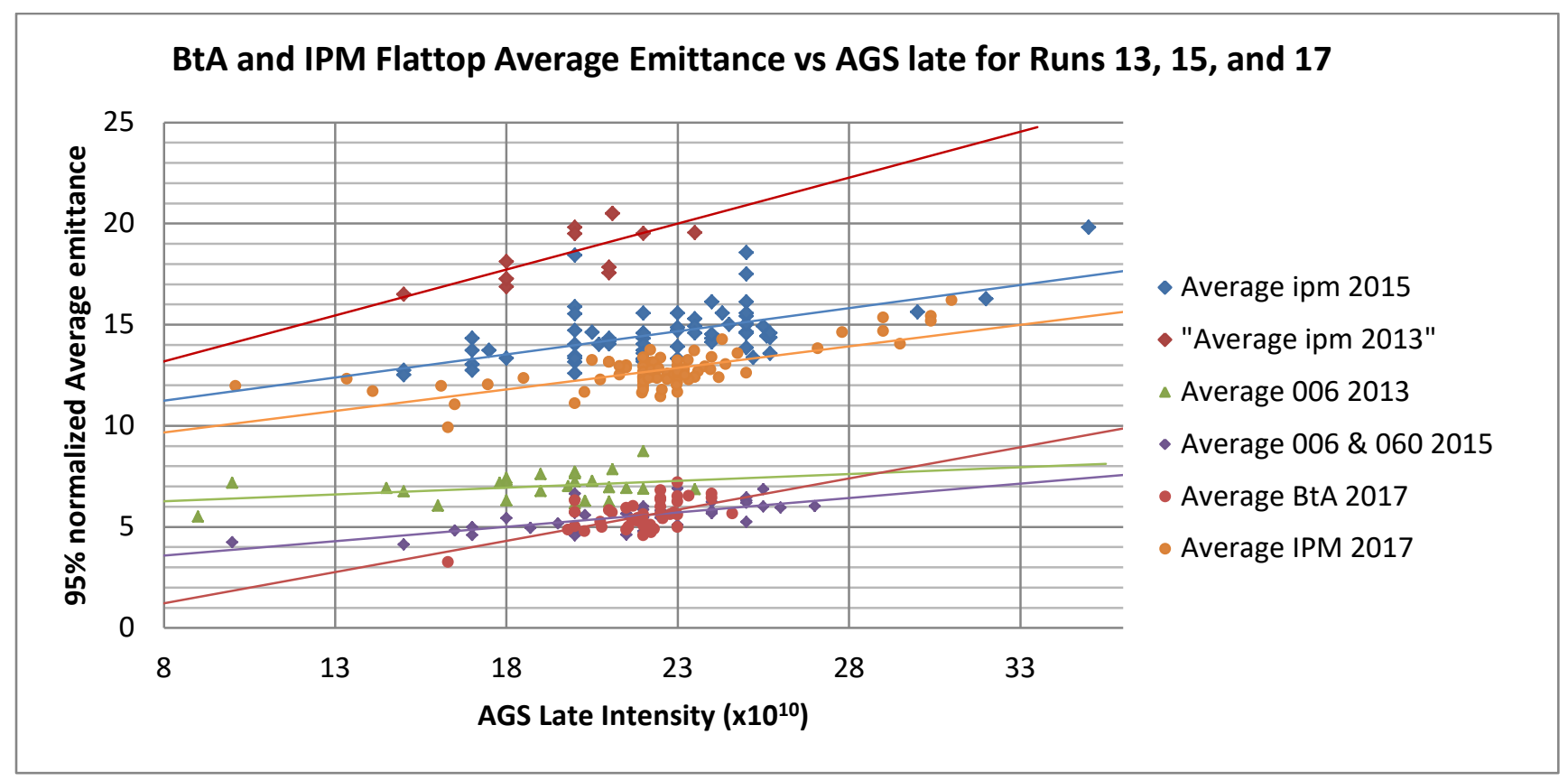

Figure 26: BtA and AGS flattop average $\varepsilon$ measurements from Runs 13, 15, and 17. From top to bottom, the top 3 sets of data are from the IPM and are from Run 13 (red), Run 15 (blue), and Run 17 (orange). The bottom 3 traces are the BtA data from Run 13 (green), Run 15 (purple), and Run 17 (red). Linear fits of the data are also included. The $\varepsilon_{\text {avg }}$ values shown here are not corrected for dispersion.

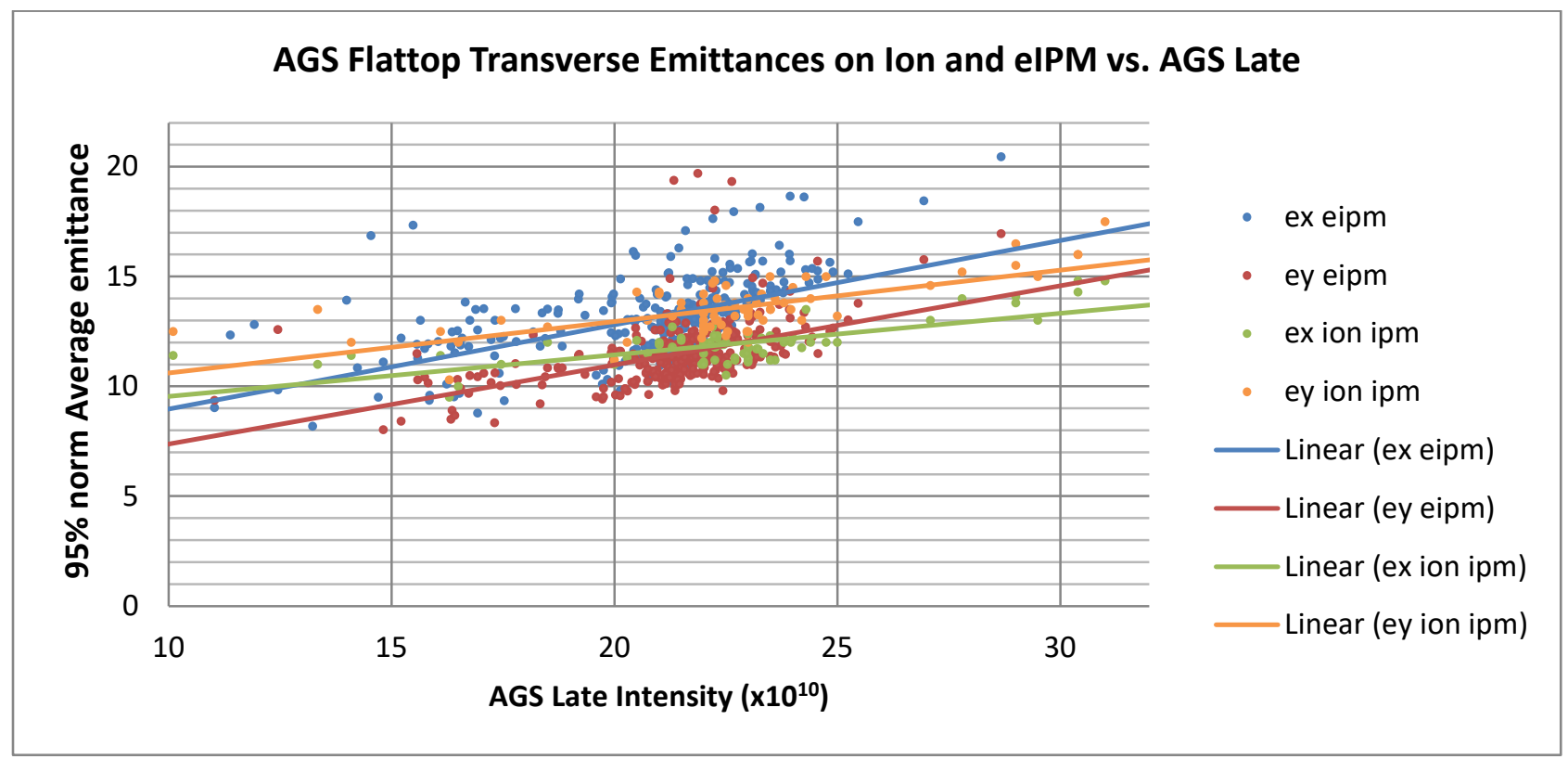

Figure 27: AGS Flattop Transverse Emittance during Run 17 using the Ion IPM and new eIPM with linear fits to the data. 
The data from March $6^{\text {th }}$ onward is with the dual harmonic on (AGS user 2). There's no obvious change in the flattop emittance with and without it and no obvious trend through the run. The average $\varepsilon_{\text {long }}$ of all the measurements is $0.92 \pm 0.07 \mathrm{ev}$-sec. $\varepsilon_{\text {long }}$ measurements in Run 15 were generally slightly larger ( 1.0 to $1.1 \mathrm{ev}-\mathrm{sec})$ although this was somewhat intentional due to the observations that wider bunches seemed to produce higher polarization. ${ }^{45}$

\begin{tabular}{|c|c|c|c|c|c|}
\hline Date & Time & Length (ns) & \# of measurements & Synch. freq. (Hz) & $\boldsymbol{\varepsilon}_{\text {long }}$ (ev-sec) \\
\hline 19-Dec & 2038 & 27.4 & 5 & 113.7 & 0.81 \\
\hline 9-Jan & 1911 & 30.44 & 5 & 118 & 1.07 \\
\hline 9-Jan & 2048 & 29.04 & 6 & 118 & 0.97 \\
\hline 17-Feb & 1901 & 29.1 & 6 & 107 & 0.86 \\
\hline 6-Mar & 2054 & 27 & 5 & 117 & 0.83 \\
\hline 21-Mar & 1427 & 27.2 & 7 & $120.5^{*}$ & 0.87 \\
\hline 24-Mar & 1755 & 27.9 & 10 & $120.5^{*}$ & 0.92 \\
\hline 4-Apr & 1223 & 27.5 & 12 & $120.5^{*}$ & 0.89 \\
\hline 25-Apr & 2046 & 28.6 & 11 & $120.5^{*}$ & 0.97 \\
\hline 22-May & 2055 & 27.8 & 8 & $120.5^{*}$ & 0.91 \\
\hline 15-Jun & 2015 & 27.9 & 10 & $120.5^{*}$ & 0.92 \\
\hline 21-Jun & 1807 & 28.5 & 11 & $120.5^{*}$ & 0.96 \\
\hline 22-Jun & 1552 & 27.9 & 6 & 120.5 & 0.92 \\
\hline Average & - & $28.2 \pm 0.91$ & - & - & $0.92 \pm 0.07$ \\
\hline
\end{tabular}

Table IX: Longitudinal emittance measurements on the AGS Flattop made during Run 17. Data is taken from the Booster-AGS-PP 2017 elog. The lengths shown are the averages of several measurements, the number of which is shown in the "\# of measurements" column. * indicates that the synch. freq. is an estimate.

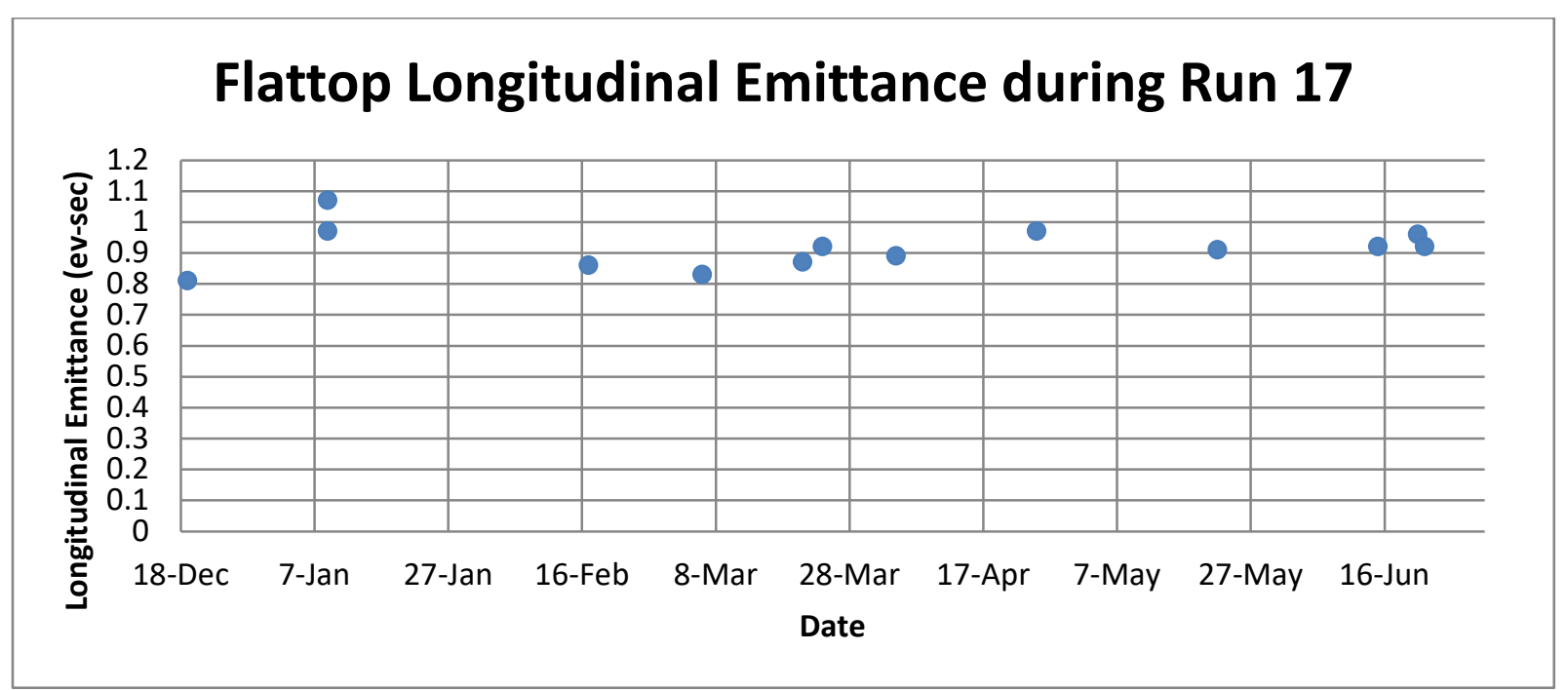

Figure 28 : Longitudinal emittance measurements on the AGS Flattop made during Run 17. Data is taken from the Booster-AGS-PP 2017 elog. ${ }^{45}$ See Booster-AGS-PP 2015 elog and "An Overview if Booster and AGS Polarized Proton Operation During Run 15",
K. Zeno, pgs 22-25. 


\section{Polarization with and without the Dual Harmonic}

The dual harmonic setup became the default setup used to fill RHIC on March $10^{\text {th }}$. The AGS polarization for RHIC fills in the 10 days following that change averaged several percent higher than it did in the 10 days preceding it. ${ }^{46}$ It's also true that the average of a set of 5 polarization measurements taken with the dual harmonic on and with an AGS late intensity of 3e11 was 70.3\%, and a measured polarization that high, with that amount of statistics, and at that high of an intensity probably never occurred before the AGS dual harmonic was implemented. ${ }^{47}$ However, when a side by side comparison was done on April $7^{\text {th }}$ the difference in polarization between the two setups wasn't so obvious. ${ }^{48}$

On that day the polarization at the normal intensity, about 2.2e11, and around 3.0e11 were measured. A constant amount of Booster scraping was employed and the input intensity was the nominal 7e11 for a $300 \mu$ s pulse. The input was varied by changing the Linac pulse length. With the amount of scraping present a pulse length of $200 \mu$ s or so provided an AGS late intensity of about 2.2e11 and a pulse length of $300 \mu$ s provided an AGS late intensity of about 3.0e11. This entails that the $\varepsilon$ of the beam injected into the AGS for both cases should be about the same so that any difference in AGS polarization between the 2 cases should be related to differences in the AGS setup. Note that under typical running conditions, the nominal intensity was provided using a $300 \mu$ s pulse and more scraping than employed in this study.

Table X shows the results. The setup with the dual harmonic is on AGS user 2 and the one without it was on user 4 . Five standard measurements were taken for each case except for the user 2 case with nominal intensity where only 4 are available. The source polarization is also included. Since the source polarization is measured on a different Linac user it should not be affected by the pulse length change. The average of the lower and higher intensity cases is essentially the same on both users (69.1\% on user 2 and $68.9 \%$ on user 4 ). User 2 seems to show more intensity dependence, but it seems doubtful that this difference is significant especially since the intensity is slightly higher for user 2 than for user 4 in the $300 \mu$ s case. The errors for each set of 5 measurements taken as a whole are in the $1 \%$ range.

What does seem significant is that the variation over the AGS measurements is greater for the higher intensity cases than for the lower intensity cases regardless of the user. In the lower intensity case the standard deviation of the measurements is $1.13 \%$ for user 2 and $1.65 \%$ for user 4 , but in the higher intensity case they are both about $2.7 \%$. As expected, the $\sigma$ of the

\footnotetext{
${ }^{46}$ See H. Huang's "Injectors: Run 17 and Beyond" presentation at the RHIC retreat.

${ }^{47}$ See H. Huang's "Injectors: Run 17 and Beyond" presentation at the RHIC retreat. Also, the AGS late intensity, measured at $920 \mathrm{~ms}$ was 3.0e11, but there was some loss around extraction so the intensity later on the flattop, where the polarization measurement is done, was probably more like 2.9e11. The measurement was taken on March $9^{\text {th }}$. There is also anecdotal evidence that new targets tend to give a higher polarization. Vertical target 3 was used here and it seems to have become the default target around Feb $27^{\text {th }}$, so it was not particularly new.

${ }^{48}$ See Booster-AGS-PP April 172017 elog
} 
source polarization measurements doesn't show any significant difference. One wonders if this is a real variation in AGS polarization, something about the polarization measurement itself, or both. Logged data from the IPM (Rf on) does show a lot of variation in $\varepsilon_{\mathrm{y}}$ in the user 2 case at the higher intensity. ${ }^{49}$ So, one could argue that the variation could be the reason for the large $\sigma$, except that the $\varepsilon_{\mathrm{y}}$ variation in the high intensity case for user 4 is not large and yet the $\sigma$ is just as large. $\varepsilon_{\mathrm{x}}$ is reasonably stable in both cases.

\begin{tabular}{|c|c|c|c|c|c|c|c|c|}
\hline $\begin{array}{l}\text { AGS } \\
\text { User }\end{array}$ & $\begin{array}{l}\text { Pulse } \\
\text { length } \\
(\mu \mathrm{s})\end{array}$ & $\begin{array}{l}\text { Source } \\
\text { Pol. (\%) }\end{array}$ & $\begin{array}{l}\text { Avg. } \\
\text { Source } \\
\text { Pol. (\%) }\end{array}$ & $\begin{array}{l}\text { Source } \\
\text { Pol. } \sigma \\
(\%)\end{array}$ & $\begin{array}{l}\text { AGS Pol. } \\
(\%)\end{array}$ & $\begin{array}{l}\text { Avg. AGS } \\
\text { Pol. (\%) }\end{array}$ & $\begin{array}{l}\text { AGS Pol. } \\
\sigma(\%)\end{array}$ & $\begin{array}{l}\text { AGS late } \\
\left(\times 10^{11}\right)\end{array}$ \\
\hline 2 & 190 & $\begin{array}{l}- \\
86.61 \\
83.68 \\
84.70 \\
84.11\end{array}$ & 84.77 & 1.12 & $\begin{array}{l}- \\
70.49 \\
73.10 \\
73.02 \\
73.17\end{array}$ & 72.44 & 1.13 & 2.25 \\
\hline 2 & 300 & $\begin{array}{l}85.62 \\
83.34 \\
87.14 \\
84.18 \\
83.00\end{array}$ & 84.66 & 1.54 & $\begin{array}{l}67.02 \\
67.05 \\
69.19 \\
63.94 \\
61.64\end{array}$ & 65.77 & 2.66 & 3.12 \\
\hline 4 & 200 & $\begin{array}{l}85.92 \\
84.37 \\
86.54 \\
84.81 \\
88.48\end{array}$ & 86.02 & 1.45 & $\begin{array}{l}68.84 \\
71.49 \\
69.12 \\
73.21 \\
71.58\end{array}$ & 70.85 & 1.65 & 2.2 \\
\hline 4 & 300 & $\begin{array}{l}85.36 \\
85.09 \\
88.27 \\
84.74 \\
83.05\end{array}$ & 85.30 & 1.69 & $\begin{array}{l}63.90 \\
70.96 \\
69.16 \\
64.17 \\
66.57\end{array}$ & 66.95 & 2.76 & 3.01 \\
\hline
\end{tabular}

Table X: Polarization measurements with (user 2) and without (user 4) the AGS dual harmonic for 2 different AGS late intensities. For each case, the “Avg. Source Polarization” is the average of the source polarization measurements and "Source Pol. $\sigma$ " is the standard deviation of those measurements and similarly for the AGS flattop measurements.

\section{Bucket Switch in the Booster to be used with a 2 to 1 Bunch Merge in the AGS}

In order to reduce the intensity dependence of the polarization and transverse emittance measured on the AGS flattop it was proposed that the AGS accelerate two bunches each of half intensity to the flattop and then merge them into one. ${ }^{50}$ Because of the Rf frequency range in the AGS, in order to perform that merge the AGS would need to accelerate on $\mathrm{h}=12$ and $\mathrm{h}=6$ would be used to merge the two bunches into one on the flattop. The beam would come from one Linac pulse, which would be accelerated in $\mathrm{h}=2$ buckets in the Booster. Since the AGS is four times the circumference of the Booster, in order for multiple Booster bunches to be injected properly into

\footnotetext{
${ }^{49}$ The data is gpm.AgsIpmEmitH_ExtAvg:dataM and gpm.AgslpmEmitV_ExtAvg:dataM in the log Ags/IPM/AGSIPM_AvgEmitInjExtAvg.lvdsp.

${ }^{50} \mathrm{H}$. Huang, private communication
} 
AGS buckets the AGS harmonic has to be four times the Booster harmonic. Clearly, this condition is not met if the Booster harmonic is 2 and the AGS harmonic is 12.

If the Booster harmonic was three but only two of the buckets were filled then the condition would be met. Because of the flexibility of the low level Rf system this can be accomplished in the following way: Capture and accelerate on $\mathrm{h}=2$, at some point start to merge the 2 bunches into 1, when the spacing between the 2 bunches is the same as the spacing between two $\mathrm{h}=3$ buckets bring on the $\mathrm{h}=3$ voltage and bring down the $\mathrm{h}=2$ and 1 voltages. With proper phasing those 2 bunches will wind up in $\mathrm{h}=3$ buckets, at which point they can be accelerated to extraction energy and transferred to the AGS. The cavity voltages and timing used to do this are shown in Figure 29.

There are other conditions that also have to be met:

1. The bucket shift has to occur when the magnetic field is relatively flat so a porch needs to be put into the main magnet function. That porch should not occur near the $\mathrm{G} \gamma=3$ and 4 imperfection resonances where $\mathrm{f}_{\mathrm{rev}}=1.19$ and $1.33 \mathrm{MHz}$, respectively. The B fields corresponding to $\mathrm{G} \gamma=3$ and 4 are 3.06 and $4.55 \mathrm{kG}$, respectively. It also needs to occur before the synchro loop becomes active (15 ms before extraction).

2. The porch needs to be long enough not only to allow time for the voltages to ramp but also to give sufficient time for $\mathrm{A} 3$ to switch from $\mathrm{h}=2$ to 3 .

3. After the bucket switch, the $\mathrm{dB} / \mathrm{dt}$ must remain relatively low until $\mathrm{A} 3$ voltage is available on $\mathrm{h}=3$.

4. The AC phase loop and Rf track are put in hold during the bucket switch and then come out of hold afterwards.

A Booster magnet cycle was constructed which met these conditions. For this particular case, the required $\mathrm{Rf}$ frequencies are within the cavity frequency ranges for all the cavities throughout the cycle so those ranges did not constrain where the bucket switch could occur.

Additionally, the time in the Booster cycle when extraction occurs and the relative timing between the Booster and AGS was adjusted so that injection into the AGS occurs at the normal time. In this way, the nominal AGS magnet cycle and timing could still be used. The setup used Booster user 5 and AGS user 6.

Figure 30 is a mountain range display of the wall current monitor during the bucket switch. ${ }^{51}$ At the bottom of the display the two bunches are in $\mathrm{h}=2$ buckets and at the top they are in two of the three $\mathrm{h}=3$ buckets. These two bunches were injected into two adjacent $\mathrm{h}=12$ buckets (see Figure 31).

${ }^{51}$ See Booster-AGS-PP-April 2020172023 entry 


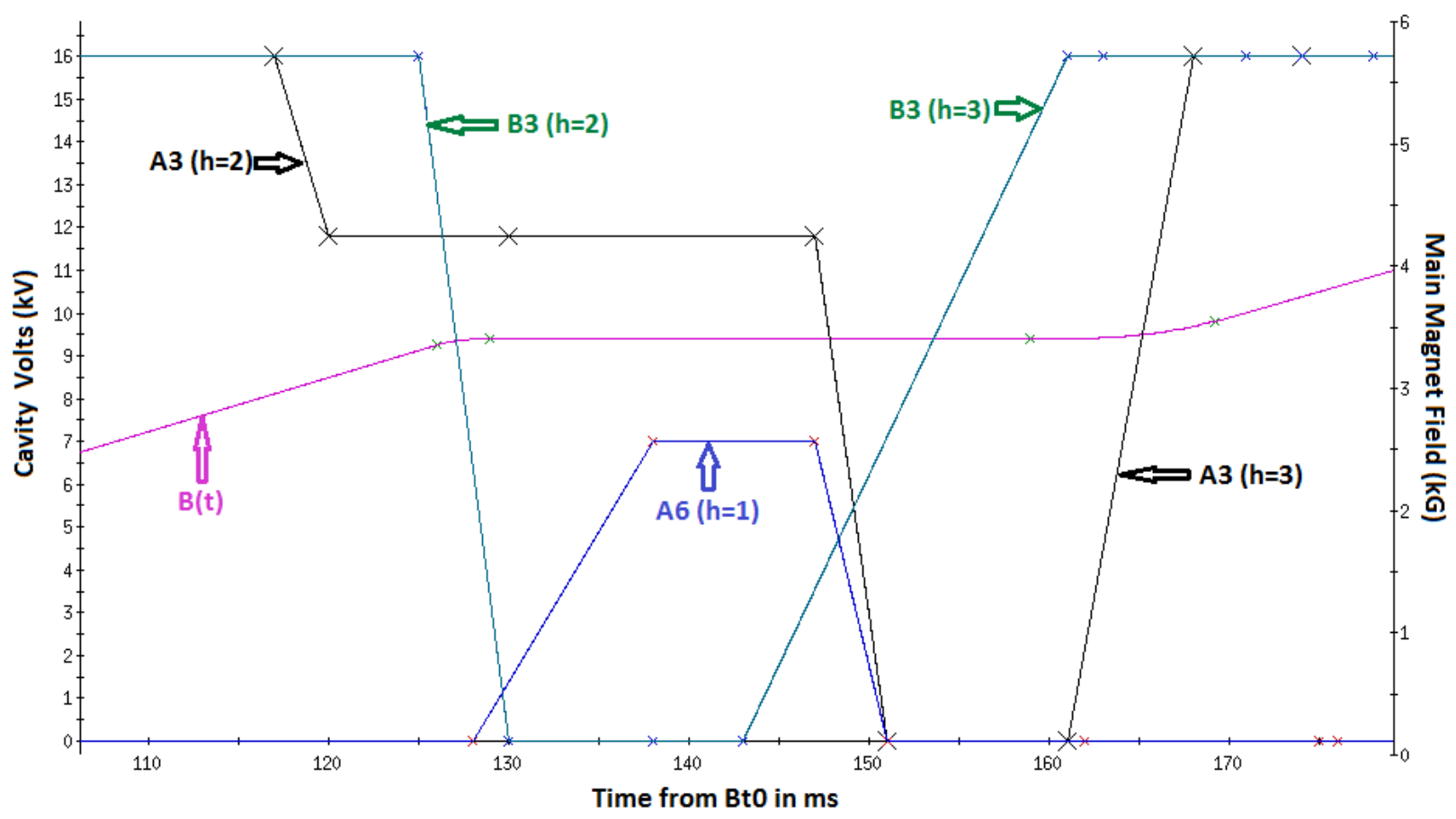

Figure 29: Rf voltage functions and harmonics used to move the two $\mathrm{h}=2$ bunches into two $\mathrm{h}=3$ buckets. The main magnet field is also show and is associated with the y-axis on the right. ${ }^{52}$

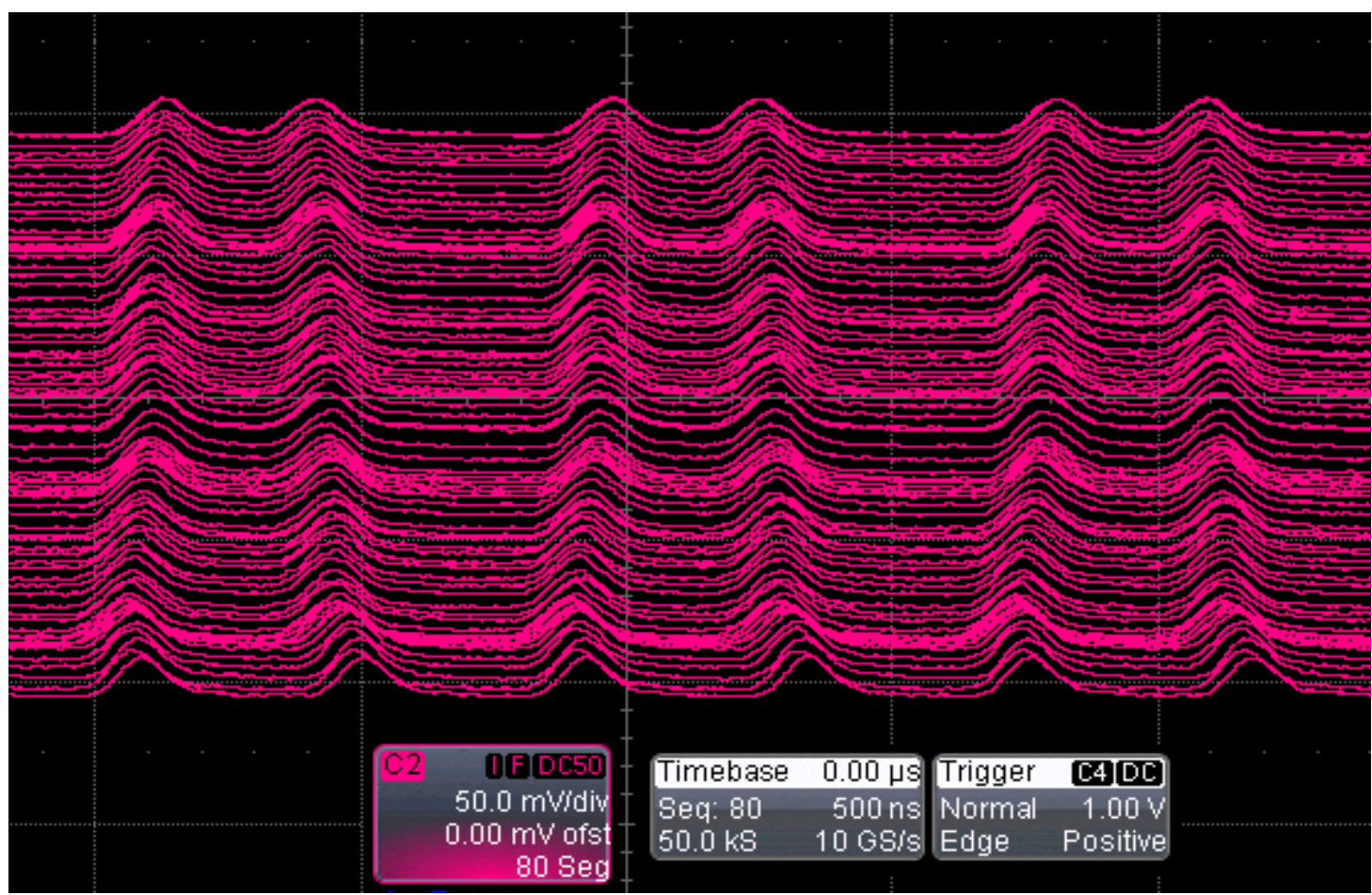

Figure 30: A mountain range display of the Booster wall current monitor during the period where the two bunches are moved from $\mathrm{h}=2$ into $\mathrm{h}=3$ buckets.

${ }^{52}$ Taken from the May 8, 20171850 user 5 archives of Rf voltage functions. 

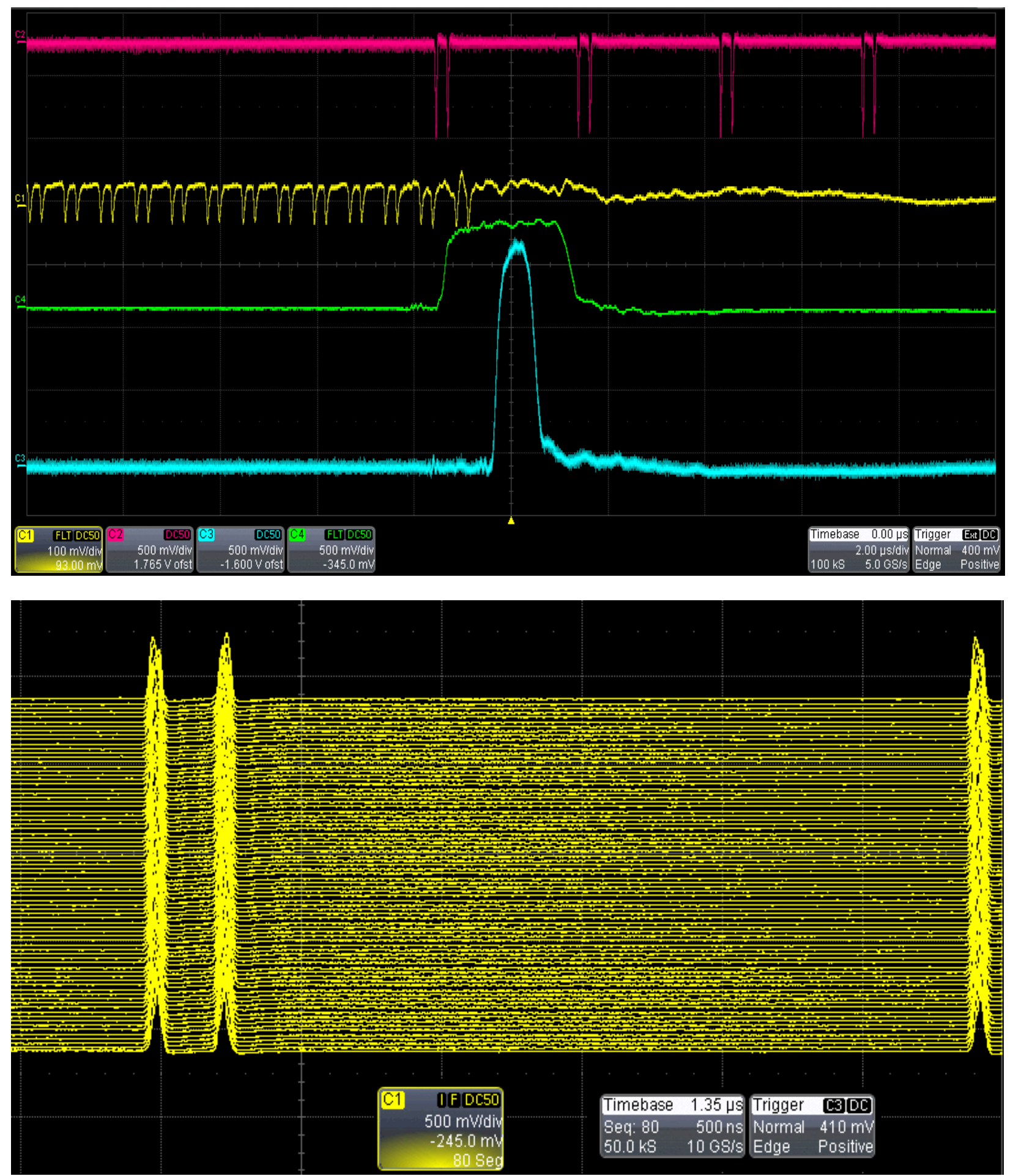

Figure 31: The 2 bunches in $\mathrm{h}=3$ buckets in Booster injected into adjacent $\mathrm{h}=12$ buckets in the AGS. The top scope picture shows the AGS wall current monitor (red), the Booster wall current monitor (yellow), the F3 extraction kicker (green), and the A5 injection kicker (blue) at the transfer from the Booster to AGS. The bottom plot is a mountain range display of the AGS wall current monitor just after injection into the AGS. 
Although this maneuver was largely successful, the goal is to produce a single bunch on the AGS flattop of relatively high intensity (say 2.5e11) with higher polarization and lower emittance than the same intensity bunch with the nominal setup. Getting enough of the AGS Rf cavities to stay on and behave normally at a high enough voltage on $\mathrm{h}=12$ through the acceleration ramp turned out to be an issue, though given enough setup time it is unlikely that this would be a significant problem in the future. Although beam was eventually accelerated to the flattop, the 2 to 1 merge there was not attempted.

If $\mathrm{h}=2$ is used for acceleration it is not possible to use a dual harmonic in the Booster because of the limited frequency ranges of the cavities. Although not fully optimized, at the nominal input intensity (7e11), there was a slow loss for the first $20 \mathrm{~ms}$ or so of the Booster cycle resulting in about $15 \%$ beam loss. With the beam scraped down to provide a Booster late of about 2.6e11 the emittances on BtA mw006 were $\varepsilon_{\mathrm{x}}=9.0$ (uncorrected for dispersion) and $\varepsilon_{\mathrm{y}}=3.2$ $\pi \mathrm{mm} \mathrm{mr}$, for an $\varepsilon_{\text {avg }}$ of $6.7 \pi \mathrm{mm} \mathrm{mr} .{ }^{53}$ Judging from Figure 24 one might expect $\varepsilon_{\text {avg }}$ for that Booster late to be around 5.0 to $5.5 \pi \mathrm{mm}$ mr. For an AGS late intensity of about 1.7e11 the $\varepsilon$ measured by the AGS IPM was about $\left(\varepsilon_{x}, \varepsilon_{y}\right)=(13,18) \pi \mathrm{mm} \mathrm{mr}$, which is much larger than with the standard setup, although it is likely that this could be improved significantly. ${ }^{54}$

Four AGS polarization measurements taken with an AGS late of about 1.8e11 were $65.98 \%, 71.16 \%, 68.12 \%$, and $62.24 \%$. The average of values is somewhat lower than what would be expected for that intensity with the normal setup. Once again though, this setup was far from optimized. ${ }^{55}$

Three bunch width measurements were made on the AGS flattop, and although the bunches had significant structure their width averaged around $19 \mathrm{~ns} .{ }^{56} \mathrm{~A}$ synchrotron frequency measurement wasn't made but the Rf voltage can be estimated from the logged vector sum and the relationship between synchrotron frequency and voltage reference found with the $\mathrm{h}=6$ setup. Using this value for the Rf voltage an $\varepsilon_{\text {long }}$ of $0.51 \mathrm{ev}-\mathrm{sec}$ was found. This is slightly more than half of the $\varepsilon_{\text {long }}$ found with the normal setup (0.92 ev-sec).

\section{Summary}

What the source(s) are of the intensity dependence of the transverse emittance as observed on the AGS flattop in the PP setup has been a long standing question. There is some intensity dependent $\varepsilon$ growth in the Booster, but distinguishing the effect of growth there on the flattop $\varepsilon$ from the effect on it from growth occurring in the AGS has been difficult. This is because the IPM measurements are not very useful early in the cycle since the lattice is distorted and also because they are not accurate when the beam is bunched.

\footnotetext{
${ }^{53}$ See Booster-AGS-PP May 92017 elog 2009 entry

${ }^{54}$ See Booster-AGS-PP May 92017 elog 2034 entry

${ }^{55}$ See Booster-AGS-PP May 92017 elog 2048 and 2057 entries

${ }^{56}$ See Booster-AGS-PP May 82017 elog 2030 entry
} 
In the AGS, a dual harmonic setup was used for the majority of the run. The purpose of this setup is to reduce the peak current during the first $100 \mathrm{~ms}$ or so of the acceleration ramp (see Figures 14-17). Measurements with and without the dual harmonic suggest that the flattop emittance is smaller with it (Figure 18). From this observation one can infer that space charge effects early in the AGS ramp are responsible for at least part of the intensity dependent emittance growth observed on the flattop.

Also, comparison of the emittances in the normal PP setup, even with the AGS dual harmonic, and the bare machine (no snakes, etc.) suggests that there is significant intensity dependent $\varepsilon$ growth in the AGS present in the PP setup that is not there in the bare machine. That intensity dependence seems to start when AGS late reaches about 1.8e11. The comparison also suggests that that intensity dependence may have a greater effect on the flattop $\varepsilon$ than the intensity dependence of the Booster $\varepsilon$ does for higher intensities (see Figure 19 and the related discussion).

Whether or not the dual harmonic improves the polarization on the flattop remains somewhat unclear to me. When looking over 20 days or so centered around the time the dual harmonic became the default setup the polarization does seem to be a few percent higher after it did, however the results from a dedicated study comparing the polarization with and without it did not show a significant difference (see Table X).

The dual harmonic does a good job of making the bunch flat before the $\mathrm{F}$ to $\mathrm{P}$ transfer, but during the transfer and afterwards, when the main magnet voltage ripple is larger, it is not quite as effective (see Figures 15 and 17). It could perhaps be improved there by locking the phase of the $\mathrm{h}=12$ cavities to the synchronous phase.

The average of longitudinal emittance measurements made on the flattop throughout the run was $0.92 \pm 0.07 \mathrm{ev}$-sec (see Table IX and Figure 28). The presence of the dual harmonic did not have a noticeable effect on it.

As regards the vertical sextupole mystery, the way the sextupoles perform in a machine with no snakes, snake corrections, lower Qv, etc. was investigated and compared to the standard Run 17 PP setup:

1. For the Run 17 PP setup, the measured $\xi_{\mathrm{x}}$ and $\xi_{\mathrm{y}}$ are close to zero from 350 to $500 \mathrm{~ms}$. The measured $\xi_{\mathrm{x}}$ is 4 or 5 units more positive than the OpticsControl model, and measured $\xi_{\mathrm{y}}$ is about 11 units more negative than it (Figure 4).

2. In the bare machine with no sextupole current the measured $\xi_{\mathrm{y}}$ is in fairly good agreement with the OpticsControl model (within 2 units) and the measured $\xi_{\mathrm{x}}$ is about 6 units more positive (Figure 5). So, the discrepancy between measured and O.C. model $\xi_{\mathrm{x}}$ 
is about the same between the PP (item 1) and bare machine cases, but the discrepancy in $\xi_{\mathrm{y}}$ is quite different in the 2 cases.

3. In the bare machine, a requested $\xi$ change of +8.8 units ( +1 O.C. unit) in either plane changes $\xi$ in either plane by about +7.2 units ( +0.82 O.C. units). See Figure 6 and Table III.

4. In the bare machine, a prediction for the sextupole currents required to obtain zero $\xi$ in both planes from the data referred to in item 3 was made. When compared to the Run 17 currents for (measured) near zero chromaticities, the predicted horizontal sextupole current is not nearly as far off from the actual as the predicted vertical sextupole current is (see Figure 9).

This suggests that there is a difference between how the chromaticites behave in the bare and the normal Run 17 PP setups for given sextupole currents, and that most notably more vertical sextupole current is required to get zero chromaticities in the Run 17 PP case. In addition, in Run 15 somewhat less vertical sextupole current was required than in the bare machine case to obtain them.

Unfortunately, the basis of these rather odd results rests to a large extent on the validity of the predictions for the currents required for zero chromaticities for the bare machine and those predictions were not verified by measuring the actual chromaticites with those settings. But regardless of the validity of those predictions, even though both strings of sextupoles are perhaps a little weaker than they're expected to be, their relative strengths appear to be very nearly the same (see Table III and Figure 7). So, the idea that the vertical sextupoles are not as strong as they used to be is not supported by the bare machine data.

The polarity and the effect on the tune of the individual sextupole magnets were also measured by measuring the relevant tune with and without a local bump at each magnet while the relevant string had current in it (see Figure 10). All the polarities looked OK, although the average tune change between the two states for the vertical sextupoles was less than it was for the horizontal sextupoles $(0.024$ vs. 0.034$)$. Whether this difference in the average tune change between the 2 strings is indicative of a difference in their relative strength is not clear, but the bare machine chromaticity measurements discussed above do not support that idea.

Another measurement of $\xi_{\mathrm{y}}$ at injection energy in a bare machine was only +1.5 for a setting of +3.0 (in O.C. units). With the sextupole settings used for that measurement, $\left(\mathrm{I}_{\mathrm{x}}, \mathrm{I}_{\mathrm{y}}\right)=(50 \mathrm{~A}, 75 \mathrm{~A})$, it was noticed that the decoherence time varied with radius and was longest when the radius was close to zero (see Table V). With no sextupole current a radial dependence was not apparent. Although this behavior may seem odd, it is not known if it has always been there or if it is a new development. It may have been the result of some instability, or it could mean that there's a significant octupole field created by some of the sextupoles (the mechanism by which an octupole field could be created is unclear). If it's the latter, and it wasn't there in Run 15, it could perhaps somehow explain the discrepancy between past runs and this year. 
A couple of studies were performed to determine if scraping at Booster injection provides a smaller transverse emittance in BtA than scraping later in the cycle. If it was just as effective, scraping early in the cycle would be preferable because it reduces activation from beam loss. Although on the other hand, scraping right at injection makes it more difficult to diagnose problems and maintain optimal injection.

The first study, performed very early in the run, only looked at scraping in one plane at a time and found that scraping horizontally at injection and vertically late in the cycle were equally effective at reducing $\varepsilon_{\text {avg }}$ in BtA and more effective than the other two ways (see Table I). Scraping vertically at injection was least effective.

A couple weeks later scraping horizontally at injection was compared with scraping in both planes later in the cycle. The latter method was much more effective (see Table II). In hindsight, it would've been sensible to also compare both scraping in both planes at injection and scraping horizontally at injection and vertically later to scraping in both planes later in the cycle. The latter comparison might be of particular interest since vertical scraping at injection is the least effective and horizontal scraping, at least in the initial study, seemed as effective as scraping vertically later in the cycle.

It is also notable that when the beam is scraped early in the cycle, in either plane, that tails develop on the vertical profile, and that the tails are not present when scraping occurs later in the cycle. I would think this has to do with a space charge effect early in the cycle that is not an issue later in the cycle.

For the run the scraping scheme was kept the same as the one employed in the past. That is, scraping both horizontally and vertically later in the cycle.

On April $4^{\text {th }}$ a scan of Booster input intensity vs. AGS flattop polarization was performed. The lowest stable input intensity for a $300 \mu$ s pulse was about 6e11 and it gave about 2-3\% higher polarization than the input that was used in Run 15 ( 9e11, see Table VI). Afterwards the nominal input intensity was reduced to 7e11, it had been about 8e11 prior to this.

Linac personnel made measurements of the 95\% normalized transverse emittance during the 2016 run obtaining $\left(\varepsilon_{\mathrm{x}}, \varepsilon_{\mathrm{y}}\right)=(4 \pm 1,6 \pm 1) \pi \mathrm{mm} \mathrm{mr} .{ }^{57}$ An estimate of the emittance of the beam coming into the Booster was also measured during Run 16 by measuring the profile widths on BtA multiwires for different pulse lengths and extrapolating what the emittance would be for a

${ }^{57}$ See D. Raparia's 2016 RHIC Retreat presentation, “iigh Brightness Beam for Linac” 
zero pulse length thereby obtaining $\left(\varepsilon_{\mathrm{x}}, \varepsilon_{\mathrm{y}}\right)=(5.4,5.8)$ and $\mathrm{e}_{\mathrm{avg}}=5.6 \pi \mathrm{mm}$ mrad (the vertical core is fit for these measurements). ${ }^{58}$ This is not far off from what was measured at Linac.

Two estimates for this emittance were made using the same method in Run 17, one was made with a high Linac current (11e11 for $300 \mu \mathrm{s})$ and one with a lower one (7.1e11 for $300 \mu \mathrm{s})$, see Figure 23 and Table VIII. In the lower current case, the emittances for a zero pulse width were $\left(\varepsilon_{\mathrm{x}}, \varepsilon_{\mathrm{y}}\right)=(8.5,4.7)$ and $\varepsilon_{\mathrm{avg}}=6.9 \pi \mathrm{mm} \mathrm{mrad}$ (the fits of the vertical core are used here so they can be compared to Run 16). Also, this data used horizontal widths corrected for dispersion which reduces $\varepsilon_{\mathrm{x}}$ by about $5 \%$. $\varepsilon_{\mathrm{x}}$ and therefore $\varepsilon_{\mathrm{avg}}$ from this scan are significantly larger than what was obtained in Run 16. Linac personnel did note that the emittance they measured this run was larger than what they measured in Run $16 .^{59}$

In the higher current pulse length scan the emittances grew faster than in the lower current case. In particular, the width of the vertical core grew, even though in the lower current case it did not grow appreciably. It is inferred from this that the space charge effects in the Booster were not significant with an input of 7e11, but were at 11e11 (see Figure 23).

Another type of scan was also done: A short Linac pulse was injected at various times with respect to the collapse of the injection bump, and from that the emittances of a zero length pulse were estimated. It was also observed that when the pulse had passed through the foil for more than $130 \mu$ s or so the Gaussian fit of the vertical core was no longer significantly different than the fit of the entire profile. Since the vertical profiles in LtB also have a narrow core and larger tails than Gaussian it was postulated that this shape may simply be the shape of the Linac beam. With this kind of scan the narrower core eventually disappears, but with the pulse length scan it does not. This could be because, with a pulse length scan, there is always some part of the beam injected just before the bump collapses.

The zero pulse length emittances obtained were $\varepsilon_{\mathrm{x}}=7.7, \varepsilon_{\mathrm{y}}=6.1$, and $\varepsilon_{\mathrm{avg}}=7.0 \pi \mathrm{mm} \mathrm{mrad}$, but if the vertical core fit obtained when the pulse is close to the collapse of the bump is used then $\varepsilon_{\mathrm{y}}=4.8$ and $\varepsilon_{\mathrm{avg}}=6.4 \pi \mathrm{mm} \mathrm{mr}$. These values also use a horizontal width corrected for dispersion.

During normal running, when there is both horizontal and vertical scraping in the Booster, $\varepsilon_{\mathrm{x}}$ in BtA averaged about 7 and $\varepsilon_{\mathrm{y}}$ about 2 to $3 \pi \mathrm{mm} \mathrm{mr}$ (see Figure 24). On the AGS

\footnotetext{
${ }^{58}$ See Booster-AGS-PP Jun 292016 elog 1625 entry for analysis. The data was taken on Jun 28 (1857 through2005 entries) and, unfortunately, the analysis used mw006 for the horizontal and mw060 for the vertical profile. Booster input was about $5.9 \mathrm{e} 11$ for $300 \mu$ s pulse. Even so for a $50 \mu$ s pulse on mw006 the FWHMs are $(2.70,5.84) \mathrm{mm}$ which correspond to $\left(\varepsilon_{x}, \varepsilon_{\mathrm{y}}\right)=(5.97,5.28)$ and $\mathrm{e}_{\mathrm{avg}}=5.6 \pi \mathrm{mm} \mathrm{mrad}$ and that $\varepsilon_{\mathrm{avg}}$ isn't far off from the value obtained when mw060 is used for the vertical.

${ }^{59}$ A. Zelenski, private communication.
} 
flattop, with a typical filling intensity of about 2.2-2.3e11, typical transverse emittances were about $\left(\varepsilon_{\mathrm{x}}, \varepsilon_{\mathrm{y}}\right)=(12,13.5) \pi \mathrm{mm} \mathrm{mr}$ (with the dual harmonic active).

The transverse emittance during this run and with the AGS dual harmonic active was compared to what it was in Run 15. In BtA it appears to be similar, but on the AGS flattop the average $\varepsilon$ looks to be about $2 \pi \mathrm{mm} \mathrm{mr}$ smaller this run (See Figure 26). There doesn't seem to be much difference in the intensity dependence of the flattop emittance between the two runs though which is somewhat surprising.

Flattop data from the IPM was also compared with data taken around extraction time from the eIPM (see Figure 27). For the typical filling intensity, $\varepsilon_{\mathrm{X}}$ for the IPM is about the same as $\varepsilon_{\mathrm{y}}$ for the eIPM and $\varepsilon_{\mathrm{y}}$ for the IPM is about the same as $\varepsilon_{\mathrm{x}}$ for the eIPM, so the $\varepsilon_{\text {avg }}$ is similar. Linear fits to the data indicate that the eIPM sees somewhat more intensity dependence in both planes.

Another Booster and AGS setup has been proposed in an attempt to reduce the intensity dependence of both the transverse emittance and polarization observed on the AGS flattop. It consists of capturing the injected beam in $\mathrm{h}=2$ buckets in the Booster, accelerating them in the Booster and then in the AGS to the flattop where they would be merged into one bunch.

There are doubts as to whether a proper 2 to 1 merge is feasible on the AGS flattop, but regardless, the Rf setup in the Booster required to allow for testing if it is is not straightforward. Because of the frequency ranges of the AGS cavities, $\mathrm{h}=12$ must be employed for acceleration and $h=6$ must be used together with $h=12$ to merge the 2 bunches into one on the flattop.

To allow for this a 'bucket switch' in the Booster, which takes the 2 bunches in $\mathrm{h}=2$ buckets and puts them into two $h=3$ buckets so they can be injected into adjacent $h=12$ buckets was developed and successfully tested (see Figures 29-31). Although the 2 bunches in adjacent $\mathrm{h}=12$ buckets were accelerated to the flattop, when using $\mathrm{h}=12$ the Rf cavities had tuning problems and so the merge on the AGS flattop was not pursued. However, there is no reason to think that this problem could not be surmounted given enough setup time since we have run the cavities using $\mathrm{h}=12$ many times in the past.

However, it was still possible to make some preliminary emittance and polarization measurements. Although this setup is in its infancy, for similar Booster and AGS intensities, the BtA and AGS flattop transverse emittances were significantly larger and the polarization was lower than with the normal setup. The longitudinal emittance of the 2 (unmerged) bunches on the flattop was roughly comparable to what it is in the normal setup. 


\section{Acknowledgements}

Thanks to Freddy Severino and Daryl Goldberg for help with the Rf. 